\title{
Evolution and Recovery: Adaptable Housing Reconstruction in Post Disaster Scenarios
}

Submitted in partial fulfilment of the Master of Architecture (Professional) Victoria University of Wellington, School of Architecture 
Thanks to Shenuka, Dylan, Andrew, Elle, Kellie and Nelson.

And Mum and Dad. 


\section{Contents}

\begin{tabular}{|c|c|}
\hline Abstract & 7 \\
\hline Chapter 1 - Introduction & 9 \\
\hline Problem Statement & 9 \\
\hline Research Aim & 10 \\
\hline Scope of Research & 11 \\
\hline Overview of Research & 13 \\
\hline Chapter 2-Background and Review & 15 \\
\hline Introduction & 15 \\
\hline Phases of Reconstruction & 16 \\
\hline What is a Disaster? & 20 \\
\hline Phases of Post Disaster & 22 \\
\hline Priority of Needs & 28 \\
\hline Chapter 3 - Response & 34 \\
\hline Introduction & 34 \\
\hline Local and Domestic Responses & 34 \\
\hline International and NGO Responses & 37 \\
\hline The Role of the Affected & 41 \\
\hline Chapter 4 - Case Study and Construction & 45 \\
\hline Introduction & 45 \\
\hline Core Shelter: Typhoon resistant housing in the Philippines & 45 \\
\hline Modification of a Product: Düzce City, Turkey & 50 \\
\hline The Role of Materiality & 54 \\
\hline Culture, Context and its Relation to Reconstruction & 56 \\
\hline Chapter 5 - Site and Context & 59 \\
\hline Introduction & 59 \\
\hline Site Selection & 60 \\
\hline Chapter 6 - Design & 71 \\
\hline Introduction & 71 \\
\hline Concept & 71 \\
\hline Process & 74 \\
\hline Outcome - The Deployment & 75 \\
\hline The Core Shelter & 82 \\
\hline Expansion & 86 \\
\hline Chapter 7 -Summary and Conclusion & 89 \\
\hline Answer to the Research Question & 89 \\
\hline Limitations, Unanswered Questions and Implications & 90 \\
\hline References & 92 \\
\hline Appendix & 98 \\
\hline
\end{tabular}




\section{Table of Figures}

Figure 1 Makeshift tent village, Muzaffarabad Pakistan (UNHCR, 2010) 18

Figure 2 UNHCR tent village, Pakistan 2006 (UNHCR, 2010) 18

Figure 3 Emergency supply air drop after the 2010 Haiti earthquake (USAF, 2010) 18

Figure 4 Airport crowded with planes delivering relief supplies, Haiti 2010 (USAF, 2010) 18

Figure 5 Emergency shelter in Auditorium after Loma Prieta Earthquake. (FEMA, 2005) 19

Figure 6 'A Model of Recovery Activity' (Kates, Pijawka 1977) 24

Figure 7 Overlapping Phases in Recent Major Disasters (Amin \& Goldstein, 2008) 27

Figure 8 Triage categories (Heide, 1989) 28

Figure 9 The Hierarchy of Needs (Maslow, 1943) 29

Figure 10 Hakka 'courtyard' house, China (UNESCO, 2010) 32

Figure 11 Iban longhouse, Borneo(Travel Blog, 2010) 32

Figure 12 Mud house, Senegal (Lonely Planet, 2010) 33

Figure 13 Floating house on the Tonle Sap, Cambodia (Author) 33

Figure 14 Approaches to reconstruction in Gujarat (Jha et al, 2010) 40

Figure 15 Frame of a core shelter (NCIP, 2010) 46

Figure 16 Core shelter with thatch roof (NCIP, 2010) 46

Figure 17 User participation and Training Model. (Author) 47

Figure 18 Roof plan for housing core. (Diacon, 1997) 49

Figure 19 Initial design of temporary housing unit. (Bektaş, 2006) 51

Figure 20 Units modified by residents. (Bektaş, 2006) 51

Figure 21 Schematic of modified settlement, Fevzi Cakmak settlement. (Bektaş, 2006) 53

Figure 22 Schematic drawing to express modifications and spontaneously built commercial units in Fidanlik settlement. (Bektaş, 2006) 53

Figure 23 Economic loss: actual cost and as \% of GDP (Amin \& Goldstein, 2005

Figure 24 Number of people killed (by income class/disaster type), World summary 1973 2002 (UN, 2004)

Figure 25 Average temperature and rainfall of selected sites (Data from

http://www.worldclimate.com)

Figure 26 Rectangular, circular and linear type plans (Sassu, 2007) 64

Figure 27 Regional styles (Oliver, 2007) $\quad 65$

Figure 28 Religious demographics of selected cities 66

Figure 29 Satellite photos of Ankara, Turkey (Google, 2010) 67

Figure 30 Satellite photos of Bhuj, India (Google, 2010) 68

Figure 31 Satellite photos of Iriga, Philippines (Google, 2010) 69

Figure 32 Satellite photos of Rio de Janeiro, Brazil (Google, 2010) 70

$\begin{array}{ll}\text { Figure } 33 \text { Stage development of the proposed design } & 72\end{array}$

Figure 34 Early concept sketches of deployment, shelter, core and expansion phases. 73

Figure 35 Sketches of deployment alterations based on climate $\quad 74$

Figure 36 Disribution model. Manufacture at a national or regional level to enable quick $\begin{array}{ll}\text { deployment } & 76\end{array}$

$\begin{array}{ll}\text { Figure } 37 \text { Construction of the base } & 77\end{array}$

Figure 38 Panels slotting together, and into the base $\quad 78$

Figure 39 Step by step construction. Deployment put together by two or three people 78

Figure 40 Tessellation of the basic deployment to create larger shelters $\quad 80$

Figure 41 Packed down deployment. The white areas are space in between the panels 81

$\begin{array}{ll}\text { Figure } 42 \text { Small house or shelter transported to a new site } & 81\end{array}$

Figure 43 Modified core shelter, Iriga City $\quad 83$

$\begin{array}{ll}\text { Figure } 44 \text { Modified core shelter, Ankara } & 84\end{array}$

Figure 45 Modified core shelter, Bhuj $\quad 85$

Figure 46 Modified core shelter, Rio de Janeiro $\quad 85$

$\begin{array}{ll}\text { Figure } 47 \text { Speculative growth patterns. } & 87\end{array}$ 


\section{Appendix}

Figure 48 Definitions of disasters. (Vos, et al, 2010)

Figure 49 Trends in occurrence and victims (Vos et al, 2010)

Figure 50 Earthquakes

Figure 51 Cyclones

Figure 52 Flooding

102

Figure 53 Volcanoes

103

Figure 54 Köppen-Geiger Climate Classification 


\section{$\underline{\text { Abstract }}$}

Every year disasters affect hundreds of millions of people, causing damage that can take months or years to recover from. The reality of carrying out the processes of reconstruction and recreating functionality is a complex and difficult task; too often it is measured in a time period of several years. The issue to be addressed through this research is the response of the built (or rebuilt) environment to the requirements of people who have been displaced following a major disaster. This thesis develops a building typology and process that can adapt to the changing requirements of the stages of the redevelopment process in a post-disaster scenario. The research focuses on natural disasters, more vulnerable populations and regions and specifically on housing reconstruction. It explores the idea of a solution that can be applied widely, to many different climates and contexts; the research question then amounts to 'can a solution be created that can 'evolve' to meet the needs at each stage of a post disaster reconstruction scenario?'

The thesis explores existing post-disaster response and reconstruction models and discusses the focuses and priorities of each. The requirements of displaced people are studied, in terms of response by the built environment, and the benefits of staged development versus end product discussed. The roles that major groups, such as local authorities and NGOs, play in orchestrating the reconstruction process are discussed as well as the important, and sometimes overlooked, role that those affected by the disaster may have.

The discussion and research then informs the design proposal. Four sites are selected and used as parameters for developing the built response to the first stage of reconstruction. The selected sites are then used to show how a generic shelter may first be adapted to be suitable for a specific climate and context and then how they may be added to and grown to become permanent and suitable housing for the displaced people. The staged redevelopment process from a partially generic emergency deployment presented in this thesis can provide a solution, or framework for a solution, to many of the problems raised by the 
research and here, but it cannot be a solution by itself; architecture or design in post-disaster scenarios must be supported and driven heavily by planning and management from local, national and international sources to be successful and fully realised. 


\section{Chapter 1 - Introduction}

\section{Problem Statement}

Every year disasters affect hundreds of millions of people globally (Vos, Rodriguez, Below, Guha-Sapir, 2010 ${ }^{1}$ ) causing damage that can take years to recover from. The process of rebuilding and re-establishing is vitally important to the successful continuation of life, industry and growth of affected societies and communities. How well or poorly the reconstruction process is carried out has a huge impact on the recovery of other dimensions of society - the businesses and the economy, the social and cultural aspects of personal life or the continuation of utilities, services and industry - all depend directly on the existence and functionality of the built environment to support them.

The focus of this research is on the redevelopment after natural disasters. It is the case that people living in areas prone to disaster, or at least the leaders of the communities, are aware of the vulnerability of their situation and in some cases have contingency plans or guidelines in the event one should strike. However the time from recognition to event is at best measured in days or hours ${ }^{2}$ and at worst, minutes or no warning at all ${ }^{3}$. The result of this is the period immediately following a disaster is characterised by a sort of chaos as injury, even death, overwhelm emergency services; property damage ruins homes and businesses and essential services are interrupted.

But the chaos is temporary and soon gives way to a level of organisation geared towards recovery and re-establishing normality. In the first few days following the disaster this involves medical treatment, search and rescue operations and providing emergency food and shelter; seeing that people are provided with the minimum requirements for living. After this, the focus begins to shift towards more long term goals - providing temporary housing to enable people to begin to resume some sort of routine; assessing structural damage for reconstruction

\footnotetext{
${ }^{1}$ See Figure 48 page 74

2 In the case of some hurricanes, floods, blizzards

${ }^{3}$ Earthquakes, fires, landslides
} 
and demolition; re-establishing base economic and industry activities; reestablishing essential services and infrastructure; creating temporary or permanent housing for the displaced.

The issue here is that while the requirements for returning to normality are well documented, the reality of carrying out the processes, overcoming all the challenges presented and recreating functionality is a complex and difficult task; too often it is measured in a time period of several years. One of the main problems faced in post-disaster then is that when people, communities and often the greater society are in a state of limbo between disaster and normality, it has a huge negative impact on their ability to function well. Having buildings to properly function out of is the base of almost everything, from businesses to government to families; the longer the reconstruction process takes, the slower the recovery of the community will be (Dynes \& Quarantelli, 2008).

\section{$\underline{\text { Research Aim }}$}

If the slowing of redevelopment is to be avoided, then one of the issues to be addressed is the suitability of the response of the built (or rebuilt) environment to the needs of people; essentially developing a building typology and process that can 'evolve' to address the changing requirements of the stages of the redevelopment process. The idea being that if a building can be modified or added to in order to respond to the occupant's current needs, then the phenomenon of being caught in 'limbo' in development can be reduced or avoided entirely and the process of recovery can continue without being restrained by reconstruction. The solution this thesis aims for will be focused on providing the requirements associated with a dwelling particular to different socio-cultural needs. This will begin with basics like water and shelter (the primary, and common to all) and evolve to meet particular spatial needs such as the family size and fulfilling social, cultural and livelihood requirements.

This will be achieved through research into the community and individual needs of each stage of recovery, a study of the responses and reconstruction processes employed currently and an assessment of the materiality and construction 
methods employed in recent projects. Firstly, the current writing and use of various 'stage' models gives a basis for identifying a typical process of how a community goes about rebuilding itself (Quarantelli, 1995; Kates \& Pijawka, 1977) and looking at how these function, ideally and in reality, will form the basis for the critique. With this in mind, the second focus is to analyse both specific projects and general guidelines that have assisted reconstruction in past disasters to identify how and why they have been successful or not. The respondents and groups driving these efforts range widely, from local community groups to national level governmental organisations, from small groups of designers or interest groups to large multinational non-governmental organisations (NGOs). Each of these groups tends to have a different focus and priorities, different strengths and weaknesses (Hicks \& Pappas, 2006; Huber, Langsaeter, Eggenhofer, Freire, Grilo, 2008; Phister, Allen, Barath, Brandenburger, 2009; Murty, Jain, Sheth, Jaiswal, Dash, 2006) that lend to their efforts. Understanding and learning from these is what will aid future solutions. Finally, the research will look at the role of culture and context in projects and its relation to both those who are driving and funding the redevelopment and those who it is aimed at aiding, the affected people and communities. Through analysis of these factors, this research hopes to identify a more sustainable and effective solution, one that continues to fulfil needs in the years after the disaster and after reconstruction has been undertaken.

\section{$\underline{\text { Scope of Research }}$}

To effectively achieve the aim, certain research parameters must be defined. First, the scenarios that the design solution will be based around will be the result of natural disasters. This is so that the situations used are ones where the destructive force is indiscriminate in its selection of targets, as opposed to manmade environmental disasters or conflict situation where the damage can be targeted, intentionally or not, at specific groups or areas. It is also to give scenarios where the government and other interest groups are working towards the same goal of recovery, something that may not always be the case in war 
and conflict. This then lessens the already considerable political and procedural complications already associated with large bureaucracies.

Secondly, while the politics and policies play a huge role in how the reconstruction effort plays out, the research is aimed at a built solution and the specific issues of its construction and development. This study will not be focusing on redeveloping policy or setting up operational frameworks. This thesis will be aimed at creating a built solution that can a) be assembled quickly in an affected region, anywhere in the world; b) can be changed to be suited to the initial physical limitations and resources of a site and c) adapted to the specific spatial and cultural requirements of the users.

Third, for the purpose of creating a globally adaptable solution the study will focus on areas around the world that exhibit a specific variety of parameters such as: 1) a density of people, identified as being at risk to major disasters; and 2) exhibits indicators of development that place it more at risk in terms of being able to recover and rebuilt successfully - typically poverty levels above $20 \%$, a comparatively low GDP or significant areas of 'slums', occupied by poor or socially disadvantaged. These are the areas that are often worse affected by disasters, "while only 11 percent of the people exposed to natural hazards live in countries classified as low human development, they account for more than 53 percent of total recorded deaths" (Amin \& Goldstein, 2008),. Study of such situations would enable a solution that may be adapted with ease to less densely populate and economically stronger communities affected by disaster.

Fourth, it will look specifically at housing. Reconstruction efforts, such as those aimed towards redeveloping business, industry, services or community facilities, play a huge role in the recovery of the society as a whole but it is the housing situation that has the most direct impact on the humanitarian crisis. Also, because of people's reliance on their homes for basic needs and to platform social-cultural interactions, livelihood activities etc., it has unique challenges associated with it. 


\section{Overview of Research}

To achieve the aim, Chapter Two will review the literature surrounding each stage of post disaster development to provide a definition of the typical stages, as it has been studied and defined over the last few decades (Kates \& Pijawka, 1977; Quarantelli, 1995; Tienery, Lindell \& Perry, 2001; Amin \& Goldstein 2008). Further, it will address the priority of needs that each stage has, identifying the crucial aspects relating to reconstruction. This includes requirements of the individual, of the family, the community and the society as a whole; this is important to identifying a program in order to respond to the research aim. It will also outline the definitions of disaster and reconstruction, as used in this research.

Chapter Three will give a brief overview of the scope of responses in relation to housing reconstruction. This includes both domestic responses at the community, regional and national levels and the international responses that come as a part of a major disaster. In doing this, the chapter will attempt to identify; a) the motivation driving how these different groups assist in the reconstruction process; b) how this affects their process; c) the repercussions this may have on the affected population and d) what role the displaced people themselves have to play in the reconstruction efforts.

Chapter Four will look closely at selected case studies and how they have approached some of the issues related to post-disaster reconstruction. The first, the Core Shelter Project in the Philippines, is an example of user-constructed housing that utilises simple construction techniques to resist future disaster events. The second is agency built housing in Düzce, Turkey where the initial construction did not meet the basic needs of the beneficiaries. It looks at how, through necessity, the inhabitants have altered the inadequate designs to suit their daily lifestyle. The second part of the chapter will address how the selection of materials impacts the design and growth of any project as well as how reconstruction efforts respond (or don't) to the specifics of the site, context and culture and what impact this has on the success of the efforts. 
Chapter Five begins to address the thesis aim through the design of built forms. It identifies the project sites that have been chosen to provide both the necessary parameters for designing a solution, as well and an environment to test, speculatively, how the design could be adapted to these likely scenarios. It outlines why these specific sites have been chosen and what aspects and challenges they represent for reconstruction projects in terms of culture and context.

Chapter Six is on the architectural solution to the aim of the thesis. It outlines the concept behind the design and how the solution responds to the issues identified. This chapter also describes the final design and discusses how it responds as a solution to the problems of reconstruction this study raises.

Chapter Seven will discuss the question of whether a solution can adapt to different stages and needs of a post-disaster reconstruction process in relation to the design. Finally, it will cover the limitations of the research and methods used and what further research can be undertaken based on the research and conclusions drawn. 


\section{Chapter 2 - Background and Review}

\section{Introduction}

As mentioned in Chapter 1, in order to develop effective built solutions in a postdisaster scenario, the process of how a society rebuilds itself must be explored. A natural disaster event varies across a wide range of magnitudes in terms of material destruction, displacement and human injury. Some have caused relatively little injury or death, but have displaced large numbers of people from their homes temporarily or permanently, others have caused widespread fatalities and economic damages. Compounding on this is the affected populations' ability to cope with the disaster. This includes governmental preparedness; funding availability; leadership effectiveness at both national, local and community levels; NGO and international assistance levels and psychological impacts. Although there are so many variables, there are also some identified consistencies.

Identified by various sources (Kates \& Pijawka 1977; Quarantelli, 1987, 1995; Amin \& Goldstein, 2008) are a variety of typical stages that a redeveloping society or community may experience. While these are certainly not rules, they can help to identify what stages a recovering society is likely to experience, what the major issues of each stage are (and the transitions between them) and what the needs of each stage may be. The aim of Chapter 2 is to identify research and observations of post-disaster recovery and reconstruction and explore how these can inform the development of a built solution. First, it will explore the specific issue of housing redevelopment, which will be the main focus of the research, and what the identified patterns and issues are. It will then look at background information in the form of defining a disaster scenario, as well as looking at post disaster redevelopment in relation to the stages of the process. 


\section{Phases of Reconstruction}

Particularly relevant to the focus of this research and distinct to the recovery of the society is the recovery of the built environment. This has been studied particularly in relation to housing, as it is a crucial aspect of reconstruction and often represents a significant portion of the scope of rebuilding, especially in scenarios that have caused a large amount of damage, and therefore displacement of the population. While housing is certainly not the only form of built reconstruction, it is closely linked to the humanitarian crisis and to the human face of the destruction that becomes a major focus of rescue efforts and media coverage alike after a disaster. It is the great interest in the preservation and continuation of human life following disaster that drives what may be a large influx of aid and support in the initial weeks and months following. It is also one of the interests driving governments to coordinate rescue and relief efforts and large NGO's to set up large scale temporary housing facilities to give shelter and basic supplies to the displaced. However, it is this that leads to far greater coverage being given to the initial stage of the crisis; there is much more interest in restoring basic conditions than there is in setting up conditions for continuity and progressive development of a society/community (Hicks \& Pappas, 2006). It is this that creates a significant issue in the construction of permanent housing and therefore a return to normal routine and patterns as existed pre-disaster; it is this reason that prompts the aim of this research. Given below is the housing phase model outlined by Quarantelli $(1982,1995)$. Based on recorded case studies, Quarantelli noted that there were four distinct forms that sheltering and housing go through in post disaster scenarios:

Emergency sheltering: actual or potential disaster victims seeking quarters outside of their own permanent homes for short periods: hours in many cases, overnight at most.

Temporary sheltering: refers to behaviour involving more than just taking shelter elsewhere during the emergency period; it refers to peoples' temporary displacement into other quarters, with an expected short stay. Obviously, emergency and temporary sheltering shades into one another, but there are differences in the behavioural aspects that are worth distinguishing 
... emergency sheltering does not usually raise the question of where and how the displaced disaster victims will be fed, but temporary sheltering does.

Temporary housing: involves resumption of household responsibilities and activities in the new quarters. The evacuees know that the living arrangements exceed a mere emergency or temporary basis, and extend for months, if not years. ...the important point is that unlike temporary sheltering, household routines must be established.

Permanent housing: the moves involve occupying permanent, residential faculties. Unlike the shading between emergency and temporary sheltering, there is usually a sharp distinction between temporary and permanent housing.

From Quarantelli (1995)

Or, to put it in more personal terms - shelter, house, housing and home (Wilford, 2004). It is important to distinguish here that this model is not an outline of the processes redevelopment should go through, in terms of a recommendation or 'best-case' scenario. Rather, it is described as being an ideal: "[this] does not refer to desirable, but how the phenomena would look like if it existed in a pure form. Thus, the types we advance are not intended to be exact descriptions of social reality but as ways of thinking about such realities" (Quarantelli 1995).

It is these 'ideal' stages that form the basis on which to critique housing reconstruction. The issue is that there are typologies for stages and needs, and they address these; however, rarely do they account for the needs of other stages, or have an ability to adapt to changing priorities or status. Temporary housing, for example, has the facility to provide for the return to a household routine. It does not, however, have the capability to support a long term occupation, whether due to deterioration, size restraints or missing facilities; if reconstruction progresses is slowed then the occupants are limited in the recovery process by what their dwelling can provide for them. Take for example the case of the earthquake in October 2005 that struck the Kashmir region of North Pakistan, affecting millions of people in the region across Pakistan, North India and Afghanistan. The international response, particularly, was overwhelming with over 100 international NGO's involved in rescue and aid 
(Hicks \& Pappas, 2006) and over USD 6 billion in aid pledged in the first month (Halvorson \& Hamilton, 2009).

Huge refugee camps were set up, by both government and international nongovernmental organisation (INGO) sources like the Red Cross and UN, to provide shelter from the coming winter; 3 months on, hundreds of camps were sheltering around 252,000 people (Mahmood, 2007). However, further on from the event, people were still living in these shelters. As observed by Halvorson \& Hamilton (2009), “...two years after the deadly earthquake, approximately 30,000 individuals faced their third winter in a tent, while 3.5 million continued to live in temporary or semi-permanent shelters."

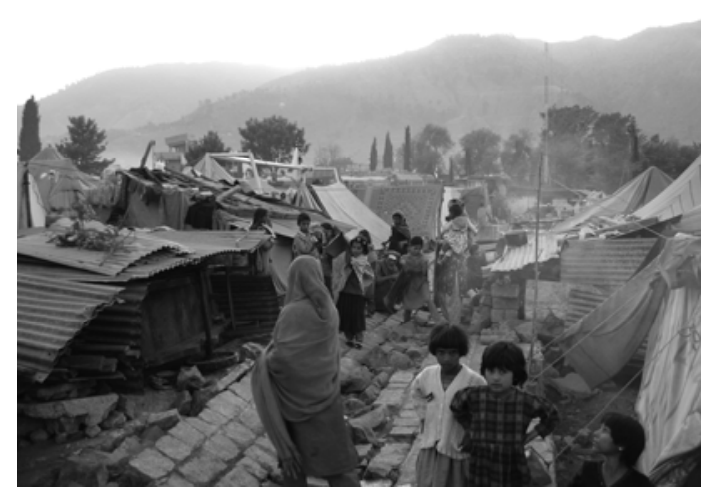

Figure 1 Makeshift tent village, Muzaffarabad Pakistan

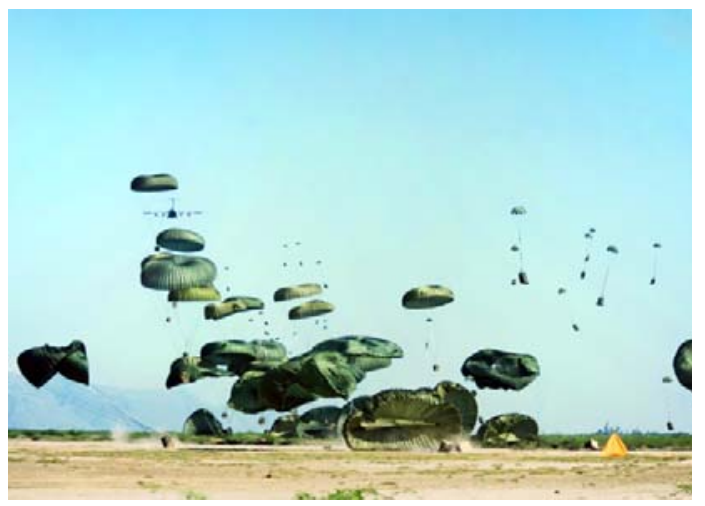

Figure 3 Emergency supply air drop after the 2010 Haiti earthquake

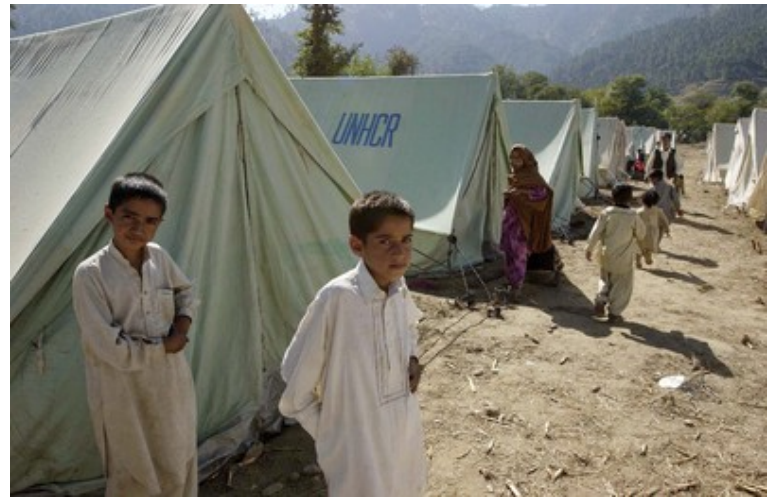

Figure 2 UNHCR tent village, Pakistan 2006

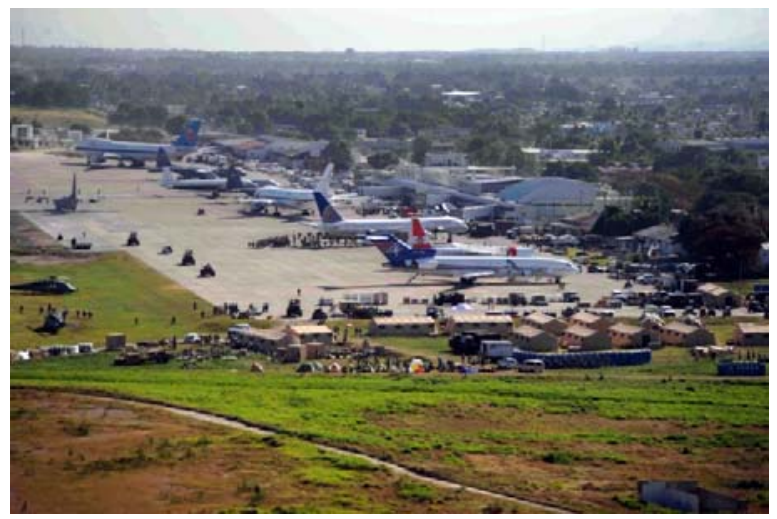

Figure 4 Airport crowded with planes delivering relief supplies, Haiti 2010 
It is difficult to address emergency shelter in a solution, due to the quick onset nature of most disasters. The provision of shelter is addressed by planning in preparation and mitigation by the community in some cases, and otherwise by whatever is available to people at the time of the disaster. In any case, the idea of emergency shelter is simply that - a minimum level of protection against the disaster and the aftermath in the hours and days following it. As noted by Quarantelli (1995), it is rarely even concerned with other provisions, like food and water, and is simply a safer place to take cover, for example, during a hurricane from the high wind and rain. Once the danger has passed, then people and organisations are able to begin search and rescue operations and damage assessments.

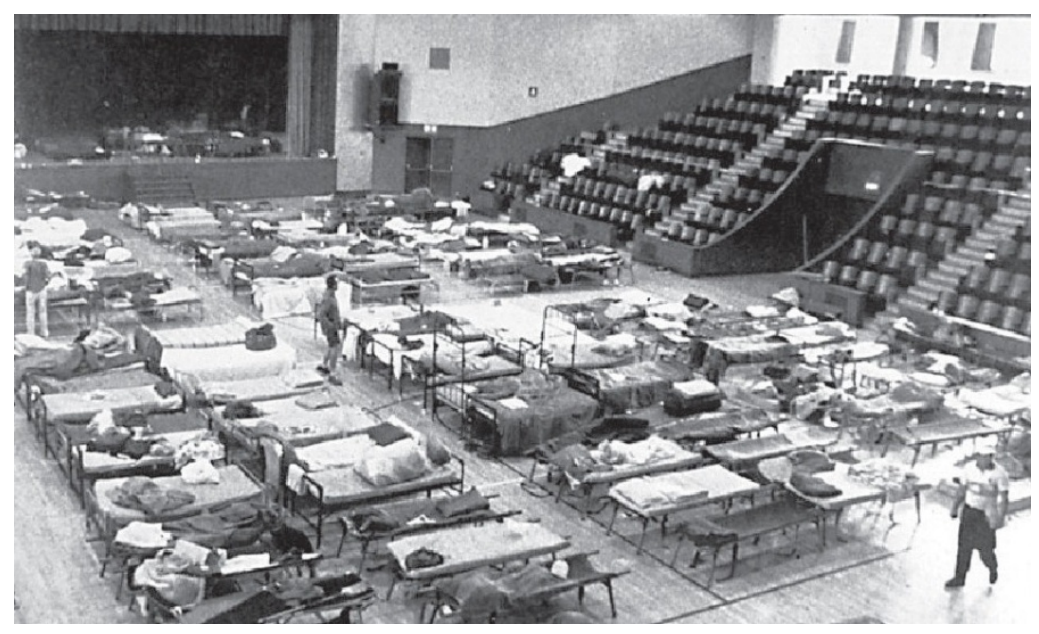

Figure 5 Emergency shelter in Auditorium after Loma Prieta Earthquake.

It is from here that the rest of the rebuilding operations begin, and it is those who have had their homes destroyed beyond repair who are the most vulnerable. 


\section{What is a Disaster?}

It is important to define exactly what the definition of a disaster is, as it will be applied to the research. One of the more widely cited definitions of a disaster by Fritz (1961) states:

"An event, concentrated in time and space, in which a society, or a relatively self-sufficient subdivision of a society, undergoes severe danger and incurs such losses to its members a physical appurtenances that the social structure is disrupted and the fulfilment of all or some of the essential functions of the society is prevented."

The key aspects contained in the definition are the concentration of danger and loss on a large population that prevents aspects of regular activities and functions and, most importantly, the presence of people - a large event that doesn't affect people significantly is not a disaster. The disruption aspect is also one of the main distinctions between an emergency and a disaster. Heide (1989) outlines a significant list of factors that separate the two events, including:

- $\quad$ Off-duty personnel called in

- $\quad$ Personnel re-assigned to new duties

- $\quad$ Everyday procedures and priorities altered

- Involvement of non-emergency responders (Red Cross etc)

- $\quad$ Non-routine tasks

This then necessitates extraordinary actions and processes in order to return the society to a functioning state again, described variously by FEMA (1984) as "an occurrence ... that cannot be managed through routine procedures" or by Tierney (1985) - "Many people trying to do quickly what they do not ordinarily do, in an environment with which they are not familiar" which gives succinct imagery of the kind of social disorder and upheaval that a major disaster can entail.

To further narrow down the scope, the research will focus on natural disasters, as opposed to manmade ones. The reason for this is that manmade disasters, such as conflict situations, can be much more complex. It is acknowledged that governments and policies play a critical role in all post-disaster reconstruction 
and the complications that arise where there is ongoing conflict or serious political disharmony within a nation is a very serious hindrance that can override nearly any amount of aid relief or reconstruction efforts. Communities displaced by war may be unable to return to their homes for long periods of time, if at all, and so makes the transition back to normality very uncertain; this makes these scenarios less appropriate for study in the context of this thesis. It is also necessary that the event not only triggers a humanitarian disaster but also property destruction, which excludes extreme temperature fluctuations, droughts and infestation/plagues. The disasters that will be the focus of the research then are what are known as sudden impact disasters and include floods, earthquakes, tropical storms, tidal waves, volcanic eruptions, and landslides (IDMC, 2006) $)^{4}$.

The other definition of a disaster here is the one related to reconstruction. Disaster recovery has changed greatly over the last 100 years as governments take more of a role and as NGO's have been created and are able to assist rapidly and globally. There is much more interest in major disasters, both on the ground and in the media, than there has been before. This is both a blessing and a curse. A disaster can see hundreds of INGOs and millions of dollars in aid poured into a region (Hicks \& Pappas, 2006; Halvorson \& Hamilton, 2009) to assist in the rescue and recovery operations. But rarely do the NGO's have a long term interest in the recovery of the communities affected; once the immediate humanitarian crisis is averted, once the media leaves, then so do the INGOs (Phister et al, 2009). They have also been criticised for not learning from previous organisational errors ${ }^{5}$, resulting in similar problems occurring repeatedly (Hicks \& Pappas, 2006; Huber et al, 2008). The government of the affected usually has a more vested interest in their recovery. To not act in the best interest of the people that they are in charge of can create a negative public opinion and can

\footnotetext{
${ }^{4}$ Specific definitions of disasters, see Figure 48

5 Noted by Huber et al (2008) there were many issues between groups in the recovery of Sumatra including the UNHIC's multi-level daily coordinating meetings were "very unwieldy" and "internal coordinating meetings were a shambles"; Coordination between INGOs and local government was an issue. Some INGOs treated Indonesia like a failing state; US agencies did not coordinate with UN. They had the perception that the UN is useless; Coordination between INGOs and Indonesian NGOs was non-existent. Indonesian NGOs were not invited to coordination meetings by INGOs.
} 
jeopardise the political future (in a democratic system) of those involved. While this push to aid recovery is usually a positive, it can create detrimental conditions. In order to maintain political favour the government has to be seen to be helping as much as they can. This motivation may see governments make grand gestures and efforts in early recovery ${ }^{6}$ and less into long term rebuilding. Some governments have also been suspected of corruption in allocating relief funds (Huber et al, 2008). A (democratic) government can only be as effective as the length of its term and a change in government can see a change in how long term recovery issues are addressed, for better or for worse. It is these sorts of bureaucratic and democratic issues that can turn disaster relief efforts into 'disasters' themselves; this is what this research aims to help alleviate, through streamlining construction through all the phases of post-disaster.

\section{Phases of Post Disaster}

One of the earlier investigations into post-disaster redevelopment was 'Reconstruction Following Disaster', by Hass, Kates and Bowden in the 1970's and published in 1977. It was undertaken based on the lack of information at the time (Mileti, Drabek, Haas 1975) of how a city goes though the reconstruction process after a major disaster and based on the observation that a disaster very rarely completely destroys a city, or causes its relocation. They state that:

"The sequence of events and processes by which a city recovers from a disaster is an ordered activity, is regular in time and space requirements, and is explainable in terms of a few significant factors:

\footnotetext{
6 In the case of the 2004 tsunami recovery in India, “...the chief minister of Tamil Nadu made a promise that everyone affected would have a temporary shelter by the prominent festival, Pongal, that was just 19 days away from the day the tsunami struck. Before making this promise, the government had neither a policy for how temporary shelters would be assigned to the affected persons nor the designs, material, or infrastructure for constructing them." This lead to a huge, rushed reconstruction effort that produced very poor quality temporary housing. (Murty et al, 2006)
} 
1. Emergency responses

2. Restoration of the restorable

3. Reconstruction of the destroyed

4. Reconstruction for commemoration, betterment and development."

(Kates, Pijawka 1977)

The model was aimed at breaking up the post-disaster period into identifiable sections, each exhibiting specific focuses. The idea being that when a disaster happened in a specific area of the world, under specific forces and being controlled by specific or localised governing factors, it could be compared and evaluated against other disasters with these stages. It outlines general activities and indicators of each sequential stage (Kates, Pijawka 1977):

Emergency Period is characterised by the society dealing with the immediate effects of the disaster; the destruction of buildings and the people who are now homeless, injured, missing or dead. It is marked by a severe upheaval of regular social and economic activities and can go on for only a few days or up to several weeks, depending on the capacity of the affected society to cope. The end of the period is typically marked by "cessation of search and rescue, drastic reduction in emergency mass feeding and housing and clearing of debris from principle arteries."

Restoration Period is focused on repairing any utilities or building that can be restored. Again, depending on the resources available, it can go on for a couple of months to over a year. Indicators of the end of the period are major roads and utilities functioning, a return of refugees and significant debris clearance.

Reconstruction Period is where buildings are replaced and economic and social activities return to pre-disaster levels. Indicators of the end are the "replacement of the population and of the functioning equivalent of their needs in homes, jobs, capital stock and urban activities." 
Commemoration, Betterment and Development Period focuses on memorializing the disaster, marking the city's improvement and serving its future development. This can extend for years or decades after.

Paraphrased (Kates, Pijawka 1977)

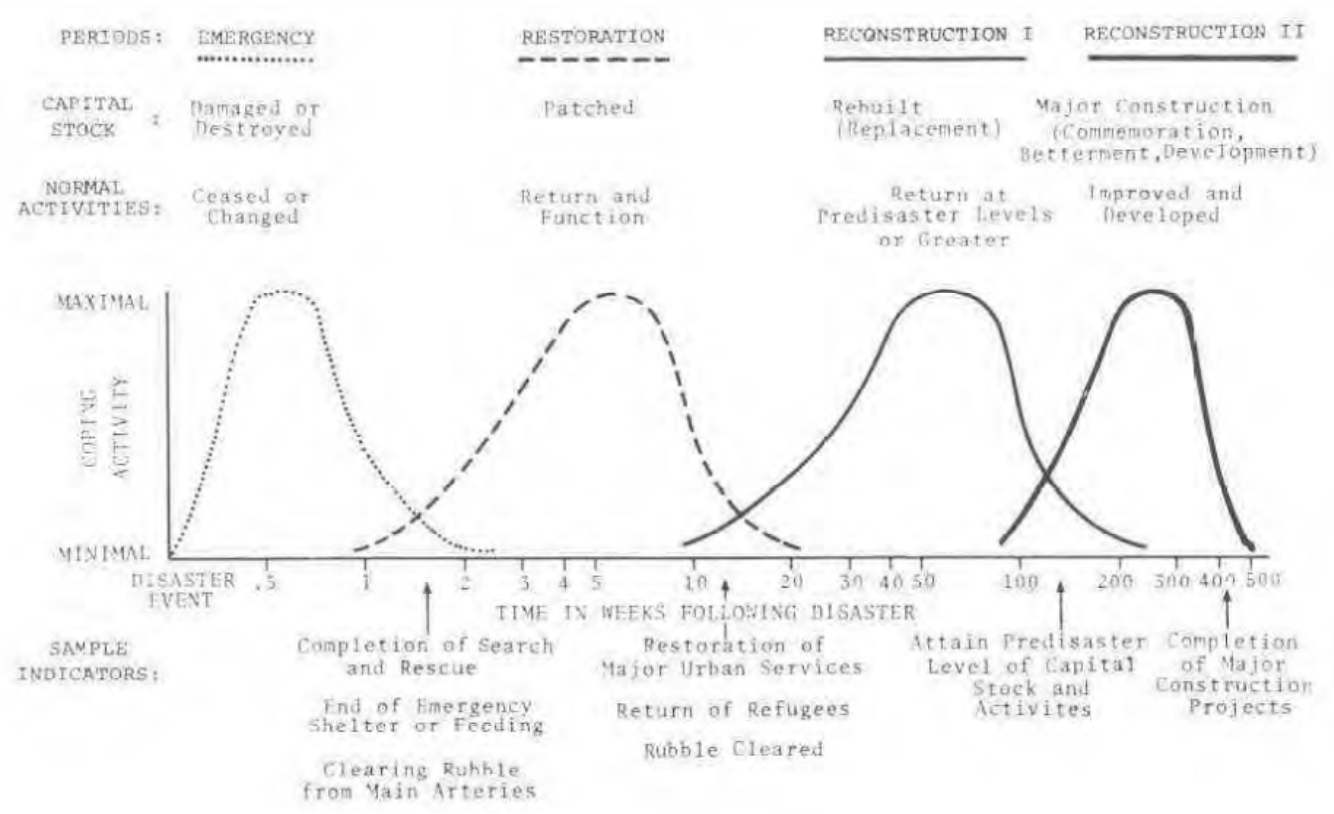

Figure 6 'A Model of Recovery Activity' (Kates, Pijawka 1977)

The United Nations International Strategy for Disaster Reduction (UNISDR) has also outlined phases of reconstruction and restoration (UN, 2004):

Preparedness involves activities and measures taken in advance to ensure effective responses to the impact of hazards, including the issuance of timely and effective early warning and the temporary evacuation of people and property from threatened locations. Early warning is not an independent phase, but an element of preparedness.

Relief or response is the provision of assistance or intervention during or immediately after a disaster to save and protect lives and meet the basic subsistence needs of people affected by the disaster. It may be of immediate, short-term, or protracted duration. 
Recovery is the set of decisions and actions taken after a disaster with a view to restoring or improving the pre disaster living conditions of the stricken community, while encouraging and facilitating necessary adjustments to reduce disaster risk. The United Nations International Strategy for Disaster Reduction includes rehabilitation and reconstruction as part of recovery.

Paraphrased UNISDR phases by de Goyet (as cited in Amin \& Goldstein, 2008)

These broad phases outlined now begin to inform how a built solution can contribute to recovery; the progression and timeline echoes the priority of needs that the people affected by the disaster are experiencing - giving an idea of the recovery effort evolves based on these.

During the first days and weeks resources are poured not into any kind of recovery or development, but are focused solely on preservation of life. The search and rescue operations trying to find any survivors and missing people; the triage process of medical care, focusing on preventing any further loss of life by treating critical injury, ways to provide survivors with the base necessities of life - food, water and basic shelter from the elements. None of these base activities are sustained for a relatively long time, nor can they be, but all are essential to setting up a base point for the society to begin the recovery process from.

The two identified models treat the next phases differently. The Kates and Pijawka model identifies repairing damaged facilities and replacing lost ones as two distinct stages whereas the UNISRD combines them as 'recovery'. Among other things, this shows the background and the aim of the authors. Kates and Pijawka, as academic researchers, are focused on providing a theoretical framework to study events on while the UN, as an NGO directly involved in disaster mitigation and recovery, focuses on the humanitarian crisis of the event and is based around the early phases of saving lives and restoring minimum levels of functionality. The consensus here is that the period is focused on 
restoring the functions and routines that are necessary for the continuation of the community. This entails providing for people something beyond simply a roof over their heads, a place where they can begin to rebuild daily activities and routines that were present before the disaster. This may sound like an obvious step, and in some ways it is, but it can be instrumental in helping restore other functions of society; it is also an important psychological step in giving people hope and vision for the future. The period also includes the return of economic activities; commercial, industrial and agricultural ventures are pivotal not only in the function of the community as a whole, but on an individual and family scale in providing a livelihood. The other major aspect here is the return of major utilities, such as water, power and sewage, all of which aid and enable the other recovery processes. ${ }^{7}$

The final phase, outlined in the Kates and Pijawka model, is the betterment of the city. This is after 'normality' has been restored to society and is focused on commemorating the event itself through memorial as well as continuing development beyond what existed pre-disaster. Both of these are important steps - the former playing both a mourning and educational role, serving as a reminder for the communities and signifying the need for preparation and mitigation; the latter marking the continuation of the society, being able to not just restore itself to functionality but to improve beyond it, with the ideal of becoming better than before. While the approach to commemoration and improvement will vary greatly depending on the scale, location and culture, the relevant aspect here is the improvement of systems in construction to resist future disasters.

The authors of the models do acknowledge some of their flaws. Kates \& Pijawka (1977) themselves state "at best, such period divisions are arbitrary, and are only useful in distinguishing major functional activities of a period," identifying that

\footnotetext{
${ }^{7}$ Housing, business and utility recovery are interlinked in recovery. "Getting business up and moving again is also critically important for resuscitating economic activities within communities that provide economic resources in the form of wages and salaries as well as goods and services. Yet, if the population lacks housing would they stay or return in the first place? ...In other words, housing recovery is critical and all types and forms of housing recovery including affordable housing are important. There is of course a chicken and egg element to this - which should come first, business recovery or housing recovery?" (Peacock et al 2007)
} 
the models do not, nor can they, take into account the large number of specific local variables in a disaster and so any identified time periods have very little use in reality. This still gives use for the models in outlining the primary needs associated with each period and a broad idea of how each phase relates to another, but even the progression of phases has been questioned by critics, as Hogg (1980) noted in her case study of the Venzone, Italy earthquake of 1976 where reconstruction took over 10 years in a stop-start manner due to external political and funding factors. This chart compiled by Amin \& Goldstein gives a visualisation of the overlapping nature of post-disaster phases.

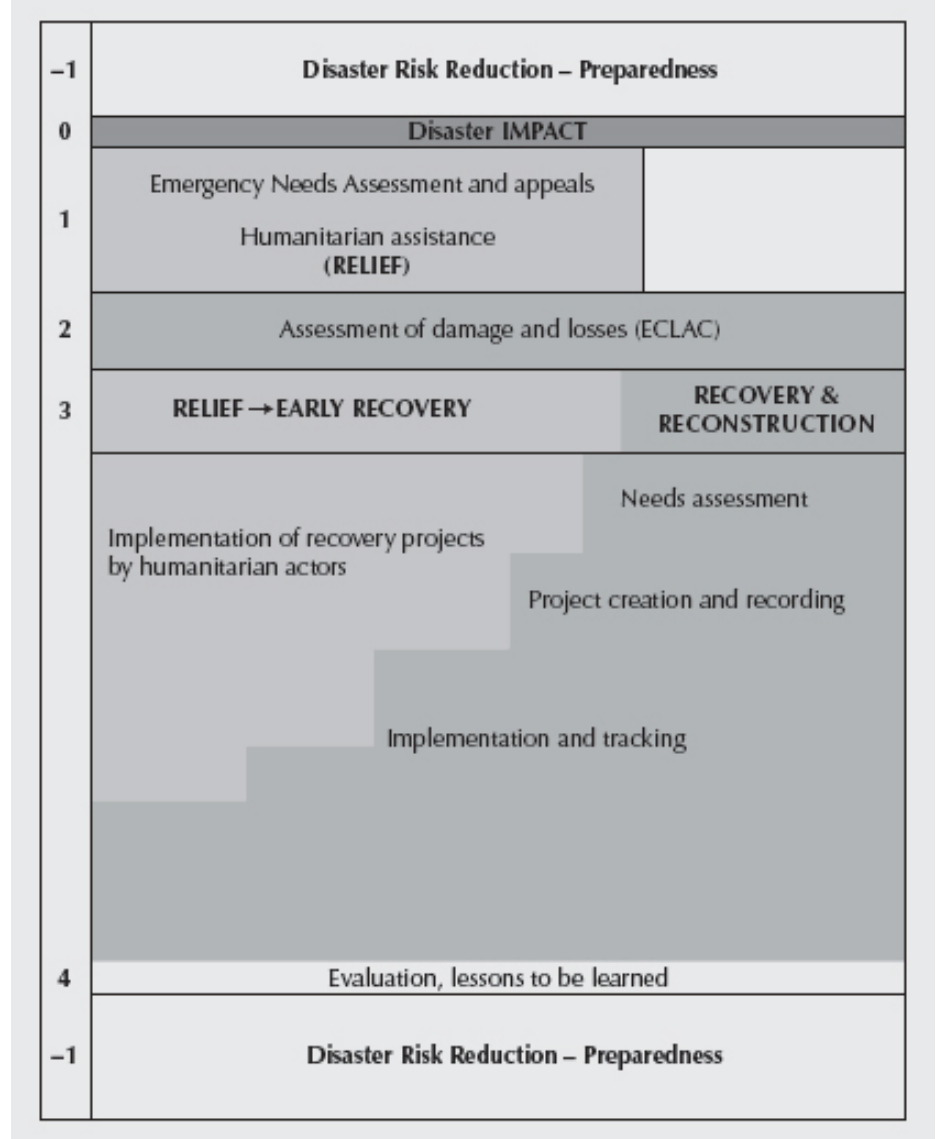

Figure 7 'The Overlapping Phases in Recent Major Disasters.' Light gray indicates that the activity is managed by the humanitarian sector. Dark gray is the developmental. 


\section{Priority of Needs}

The main reason that reconstruction and recovery tends to be a graduated progression of stages, rather than building a permanent replacement immediately, is to meet the needs of the displaced as quickly as possible. To reconstruct a functional dwelling takes time and resources, neither of which may be available immediately after. There tends to be intermediate stages to meet the immediate needs (Kates et al, 1977; Quarantelli, 1995); a permanent dwelling fulfils a wide variety of needs for the occupants, not all of which are necessary immediately. This is where prioritising becomes important.

This can be compared somewhat to the medical triage system, a separate but equally vital process in post disaster recovery. The basic concept of triage is to do the greatest good for the greatest number of casualties (Heide, 1989). This concept has created various models, all of which address the same principle of tending to life-threatening injuries first, potentially life-threatening second, nonlife threatening third and so on. This is outlined in Figure 5 below, from Heide's book 'Disaster Responses: Principles of Preparation and Coordination' (1989).

\begin{tabular}{|c|c|c|c|}
\hline PRIORITY & COLOR & SYMBOL & CASUALTY CONDITION \\
\hline FIRST & RED & $\mathrm{R}$ & $\begin{array}{l}\text { CRITICAL: likely to survive if simple* care } \\
\text { given within minutes. }\end{array}$ \\
\hline SECOND & BLUE & $\mathrm{B}$ & $\begin{array}{l}\text { CATASTROPHIC: Unlikely to survive and/or } \\
\text { extensive or complicated care needed within } \\
\text { minutes. }\end{array}$ \\
\hline THIRD & YELLOW & $\mathrm{Y}$ & $\begin{array}{l}\text { URGENT: Likely to survive if simple** care } \\
\text { given within hours. }\end{array}$ \\
\hline FOURTH & GREEN & $\mathrm{G}$ & $\begin{array}{l}\text { MINOR: likely to survive even if care delayed } \\
\text { hours to days. May be walking OR stretcher } \\
\text { cases. }\end{array}$ \\
\hline NONE & BLACK & $\mathrm{X}^{* * *}$ & DEAD \\
\hline \multicolumn{4}{|c|}{$\begin{array}{l}\text { *Simple: Care that doesn't require unusual equipment, or excessive use of time or personn } \\
\text { **Assigned THIRD priority (after YELLOWS) when there are so many casualties that if } \\
\text { resources are used in vain to try to save BLUE cases, the YELLOWS will needlessly die. }\end{array}$} \\
\hline
\end{tabular}


These four or five categories are echoed across all models; they can be outlined bluntly as cannot wait, has to wait, can wait and lost. The concept here is that while all casualties and injuries must be tended to, there are some which have much greater impact on the patient's ability to survive. These most crucial aspects must be dealt to first.

It is this concept that is applied to post-disaster recovery. Humans have a wide variety of needs, some simple and some complex, and some have a much greater bearing on survival than others. This concept as it is related to people is addressed in the fields' of psychology and sociology. Notably it was first identified in Abraham Maslow's 1943 paper entitled 'A Theory of Human Motivation' and is most often summarised in the form of a pyramid of needs (Figure 9).

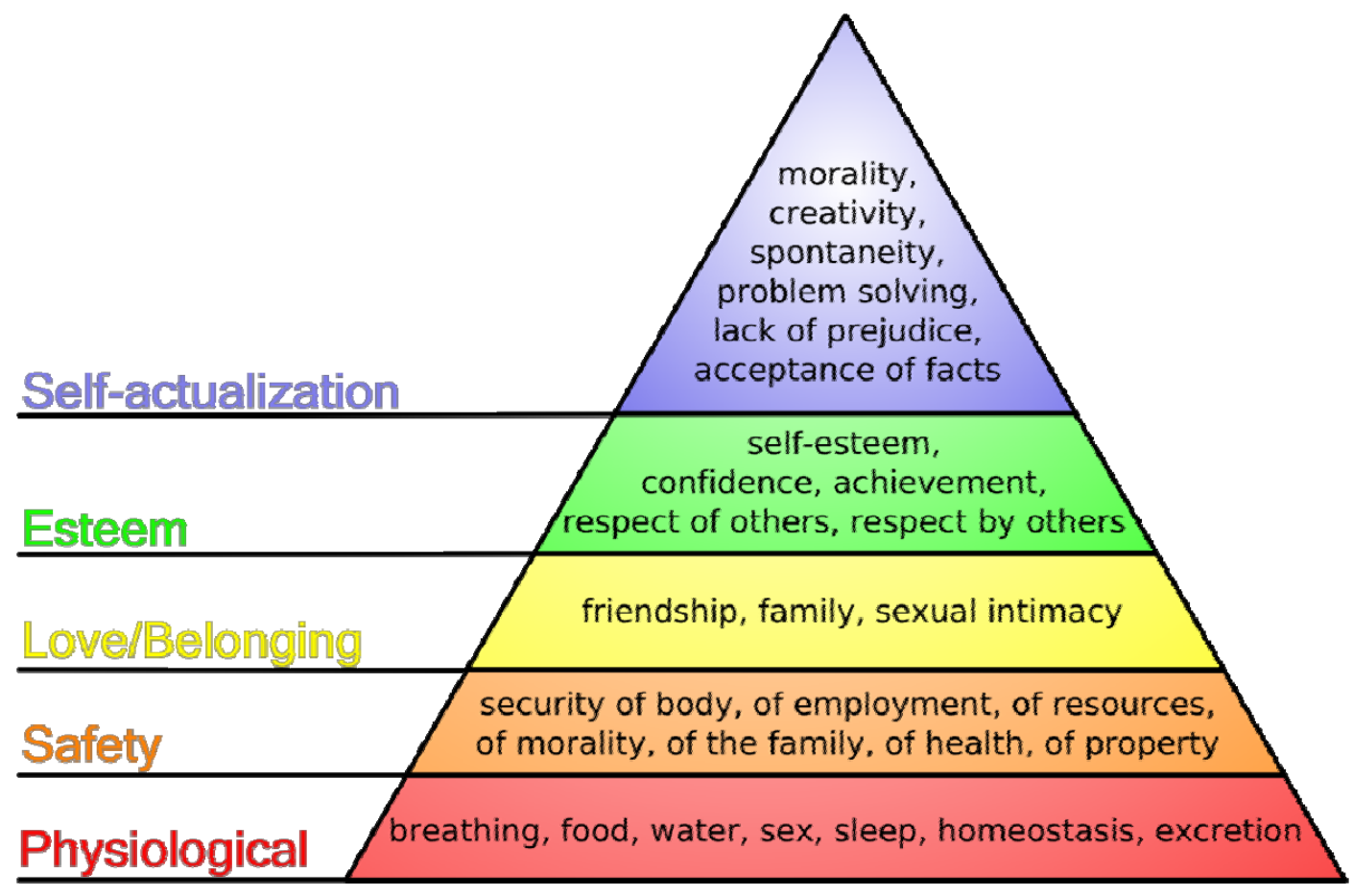

Figure 9 The Hierarchy of Needs (Maslow, 1943) 
Maslow's model has been criticised and has had other models developed to improve and correct it ${ }^{8}$. Maslow's asserted that in order to focus on needs higher up in the pyramid, the ones beneath it must be completely fulfilled, a point that has been widely criticised as being generalised and untrue in cases. Certainly sexual intimacy is not itself a prerequisite for confidence and achievement. However, beyond the scholarly psychological criticisms there is a simple model that, especially in relation to disaster recovery, gives a hierarchy that can form a base for a sort of reconstruction triage.

The use of Maslow's Hierarchy here is purely as a starting point to outline a hierarchy specific to the built environment and reconstruction. Increasing, as you move up the pyramid, the needs are less and less affected by a person's direct surroundings and more by their own notion of success and achievement. Indeed the levels of esteem and self-actualisation are defined internally by the individual and so are not as important in relation to this research. Even selections from the lower levels are outside the realm of what a dwelling usually provides (though it is not always the case), such as safety of employment.

What does apply to the home then becomes useful in identifying what is most important and why post-disaster redevelopment often occurs as outlined in the models discussed previously in the chapter. Human needs from the built environment can then be outlined as:

- Basic Physical Needs: These are what are essential to all people for living. Namely food, water, shelter and sleep.

- Cultural Needs: The expectations of these needs vary widely depending on location, social and economic status. They can include security of resources (dependable access to water, ablutions, power etc.), levels of comfort and health, quality of environment and security of property.

\footnotetext{
${ }^{8}$ Notable is Clayton Alderfer's ERG theory which takes the same hierarchy as outlined by Maslow. It divides and redistributes them into new groups - Existence, Relatedness and Growth [ERG] but also alters the theory, stating that one level is not necessarily a prerequisite of the next and that the success or failure at any level has a direct impact on the persons focus towards others. Maslow's theory is used here for the usefulness of its divisions.
} 
- Social Needs: These revolve around the interaction of family and other relationships; how these are supported and sustained by the dwelling, the daily routines within and in relation to the wider society.

Basic needs are the ones that do not vary and represent the minimum requirements for living. They apply to all people, regardless on cultural, economic or social backgrounds, and must be present. Activities considered as relief by the World Bank include search and rescue, evacuation, food and water distribution, temporary sanitation and health care, temporary shelter, and restoration of the access to transport (World Bank 1995). These are addressed by emergency relief aid-such as water, food, shelter, routine health care, and disease control-and continue to be addressed through temporary post-disaster measures (Amin \& Goldstein, 2008). These are not necessarily provided by facilities in early shelter measures; instead they come from external sources such as government and NGO aid stations, temporary wells and other facilities. They must eventually become a vital aspect of the dwelling, as relief and aid do not continue indefinitely. It is the case then that early dwellings provide only the basic requirement of shelter, a place to sleep and some protection from the elements. The access to water, sanitation and food preparation areas comes as the utilities and resources become available in the following months.

The concept of security of resources covers a dwellings access to these utilities. It is a condition that is dependent on the background of the affected; what the conditions were before the event. Some groups may consider in-home access to water and power as a basic requirement, while it is not the case for others where small community wells may be the norm. This cultural sphere of need also encompasses the configuration of the home to assist daily routine, a vague definition of activities that is again, dependent on the cultural norms present. This sweeping statement includes simple things like the configuration of food preparation spaces and their relation to eating; to more complex ones, such as the privacy of women in Muslim households (Khan \& Moore, 1990), which create a need for strong segregation of spaces within. This is also the realm of social 
needs provided by the home, how the different people and actions within relate to each other, as well as the relation to neighbours and to the wider community. The home represents a lot more than simple shelter, as noted by Barakat (2003):

"The loss of a home constitutes not just a physical deprivation, but also a loss of dignity, identity and privacy. It can cause psychological trauma, challenges perceptions of cultural identity, disrupts social structures and accepted social behaviour, poses a threat to security, and has a significant negative economic impact."

The levels and thresholds of public and private vary widely across cultures. Some exhibiting high levels of community integration, such as the Chinese Hakka and Tu lou (Figure 10) walled 'villages' or the longhouses of Borneo (Figure 11). Others live much more privately such as the nomadic herders of the Mongolian steppes in single family gers (felt tents), often far removed from towns and other families.

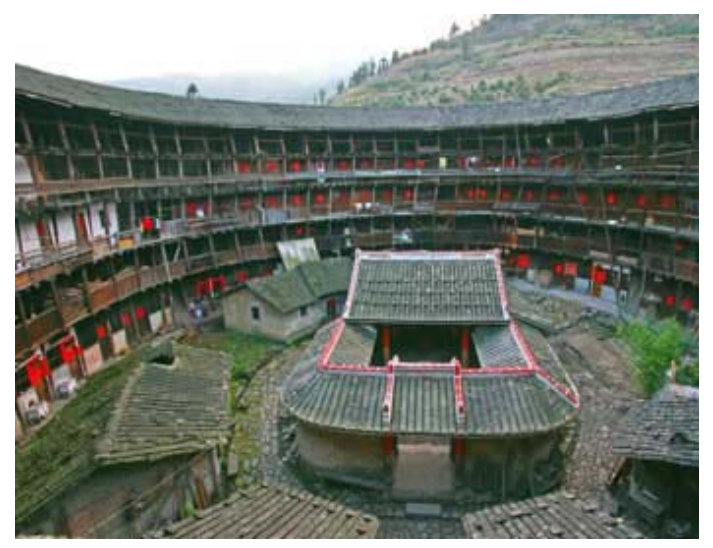

Figure 10 Hakka 'courtyard' house, China

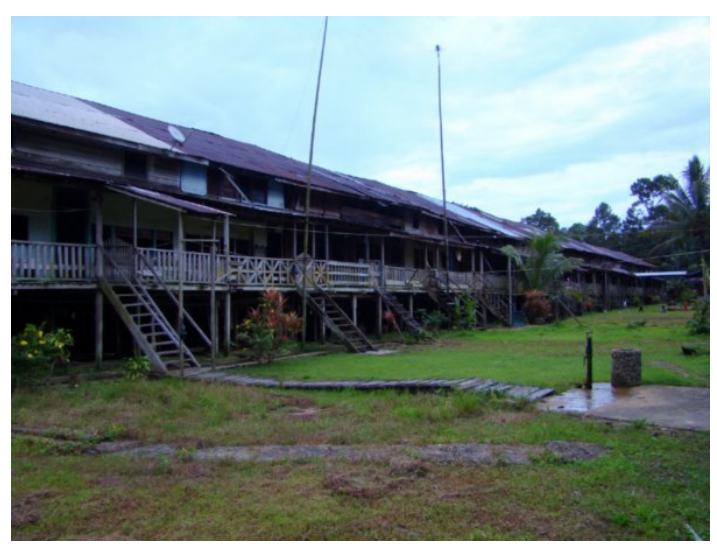

Figure 11 Iban longhouse, Borneo

(UNESCO, 2010)

Covered here are also specific climatic factors. Vernacular architecture of any region has within it specifics for dealing with the local conditions - the heavy walls and small penetrations of the desert climates of North Africa; the natural ventilation systems of traditional Malay houses; the floated pole houses of Cambodia. All measures to enable comfort and safety in regions with climatic 
extremes. It is these aspects, numerous and varying, that must also be taken into account in rebuilding to ensure success. It is this that enables a dwelling to move beyond providing temporary relief and to begin to provide a permanent functional home.

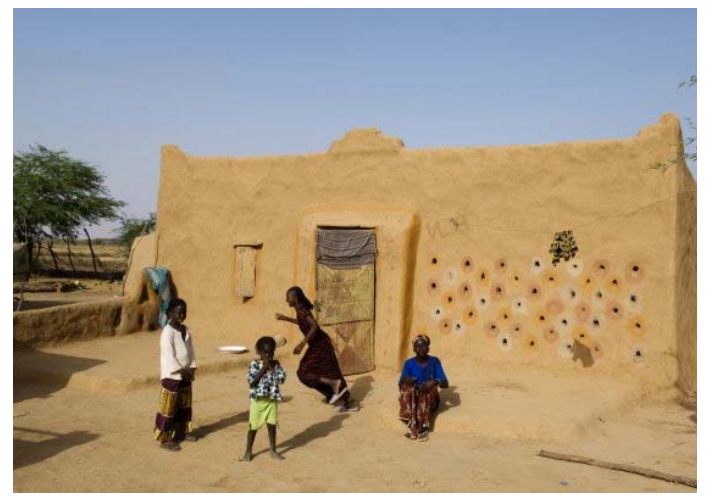

Figure 12 Mud house, Senegal

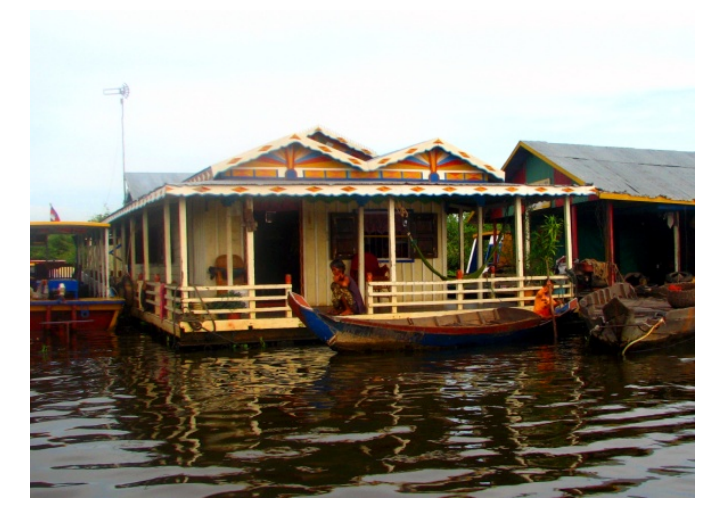

Figure 13 Floating house on the Tonle Sap,

Cambodia

The role of the house is varied. Ranging from basic shelter, an aspect covered extensively in current post-disaster models, to a permanent home, which is less so. It is the gap that sits between these two extremes that can have such an impact on the redevelopment of the community, and it is this gap which is the focus of this research. 


\section{Chapter 3 - Response}

\section{Introduction}

"Various architects, designers and other technical actors have mistakenly considered housing only as a product, but it is definitely a process" writes Baradan (2006), identifying a common failure in the post-disaster housing delivery in his case study of the response to the Turkish earthquakes of 1999. The delivery of a permanent housing solution is recognised as being more than simply creating a building, but integrating the solution with the social structure, way of life, local economy and social consciousness with the participation of the affected community (UNDRO, 1982; Barakat, 2003, Jigyasu, 2004).

This process involves many different players, both domestic and international. The affected population themselves, arguably most critically; the governing bodies, both local and national levels, and domestic NGOs. The international groups consist of the large INGOs, such as the UN, World Bank or Red Cross as well as numerous smaller INGO's.

The major issue to be addressed here is what role each of these groups is best to take in the process of housing reconstruction, if any. Each has strengths and weaknesses and identifying these is important to being able to develop a strong process. This lack of defined roles has been a recurring cause of issues encountered - poorly developed solutions by detached groups, lack of management and support from governments or a lack of sensitivity to local culture or conditions (Wahlroos, 1999; Barakat, 2003).

\section{Local and Domestic Responses}

The initial stages of the disaster recovery are the most vulnerable time for the affected. The phases of emergency and temporary shelter span a time of uncertainty for individuals and families. During this time, the extent of the damage is assessed and those who are unable to return to their former dwellings 
become the displaced - the human focus of this research. Of these displaced, it is those who lack financial or social assets who are the most vulnerable of all and it is these people who turn to public facilities to seek shelter; those with more resources are more likely to shelter with friends and family or be able to temporarily rent while displaced (Peacock, Dash, Zhang, 2007).

So, the humanitarian response becomes critical, especially in areas with a high population of disadvantaged or poor. In media coverage, and indeed in reality, it is seen to be the large INGOs who take command of this relief effort. It is suggested that this is not an ideal scenario:

"In any disaster-related situation, the ultimate responsibility for the coordination of humanitarian assistance is with the host nation. UN agencies and other international humanitarian organizations are there by invitation only to assist national authorities in their emergency and relief activities" (Hicks \& Pappas, 2006).

It is important for the government to be involved in the process right from the outset, as the cessation of humanitarian relief efforts will often see many INGOs leaving the disaster area. By being actively involved, the government is set up to be able to plan for recovery beyond the duration of the humanitarian efforts. It also gives them charge of the rehabilitation, instead of having to rely strongly on the management of groups who will not be involved for the long term recovery. This in turn means that without serious investment and engagement from the government, any reconstruction efforts become severely handicapped. "The basic requirement is political will," writes Wahlroos (1999), "in the absence of such will and support at both the central government level and at the municipal level, even the most advanced, radical or liberal strategies will fail." The barrier to this is that the governments of developing nations may not have the resources and finances to have the necessary control. Exemplified in recent large scale disasters $^{9}$ is the amount that governments depend on financial contributions from large NGOs and other governments; these donations and contributions are held by the World Bank which then means that financial control, and control of

\footnotetext{
${ }^{9}$ The 2004 Indian Ocean Tsunami and reconstruction in places like Sri Lanka and Sumatra; the 2005 Kashmir earthquake and the 2010 Haiti earthquake.
} 
the reconstruction, is shifted to an INGO. This can be a vicious cycle of control, or lack of, for the government and local authorities.

Beyond the initial stages, a continuing relationship between the individuals and families and the government can benefit the process of recovery. The sources of funding have an impact on how the permanent reconstruction is played out. Of particular note here is that if houses are gifted, then the recipients generally are far less invested in them. Having at least partial ownership tends to see inhabitants more invested in the care and further betterment of their houses, so the use of loans ${ }^{10}$ with adequate monitoring can be beneficial to the process. The government can subsidise certain aspects of the reconstruction, such as materials or technical advice, to give some assistance to locally driven rebuilding. This is known as 'partial contribution through self-help'. (Barakat, 2003). For example, after the earthquake in Gujarat in 2001, the government provided cash to victims who had lost their houses under the 'Building Your Own House' scheme. This option was preferred by the villagers to receiving housing from voluntary organisations, as that meant relinquishing the land they previously owned (Nikhileswarananda, 2004).

The restoration of permanent housing for all is not usually going to be a government's main concern. A community may have lost many other aspects critical to its function and restoring all these is just as important - the reestablishment of industries and commerce to provide work and supplies to the recovering communities, and the repair of damaged utilities to provide necessary services to buildings, "...reconstruction programs, if well conceived, can be an opportunity to develop building industries on a local or regional scale. It can also enhance employment with innovative techniques and lean on existing know how and develop local competences." (D'Urzo, 2002). These, in tandem with housing, are necessities for complete recovery; this is one of the reasons why individuals must be able to be involved in the housing process. "Finding ways to involve legitimate sources of local authority in any reconstruction programme is likely to be crucial, since exclusion risks a hostile reaction" (Barakat, 2003). The

\footnotetext{
${ }^{10}$ The Bangladesh based Grameen Bank is one example of using loans to assist the poor or displaced in recovery. Loans are given without collateral. Borrowers must form groups to obtain a loan (Yunus, 2007) and so peer pressure is used to ensure the repayment of loans taken.
} 
government's role in housing reconstruction must be one of support. Support in provision of materials, of funding sources or of supervision; to give the communities the resources necessary to rebuild their lost homes.

\section{International and NGO Responses}

In the event of major disasters, the response from the international community is often swift and great. Millions of dollars in aid are poured in by other governments. Hundreds of NGOs may descend to assist in the humanitarian crisis. This wide ranging response can be responsible for saving many lives and providing shelter to thousands of displaced families. It can also cause issues. The sheer number has caused problems in the coordination of individual groups in many recent disasters ${ }^{11}$ (Hicks \& Pappas, 2006; Huber et. al., 2008); many of these NGOs deal solely in humanitarian issues, not developmental (Barakat, 2003; Wahlroos, 1999) or the solutions provided are inadequate for the location or culture (Jigyasu, 2004).

Barakat observes that "...housing reconstruction is often construed as a developmental responsibility rather than properly [as] a humanitarian concern, and consequently tends to be low on the humanitarian agenda," and it is easy to see how the two are viewed as separate issues. The concern of many of these INGOs $^{12}$ such as the Red Cross (IFRC, 2010), is to prevent loss of life and injury; often done through providing emergency healthcare and shelter. However, Barakat argues that the humanitarian imperative to ensure access to water, sanitation, food and healthcare is the same as providing adequate shelter and housing and that the two cannot be viewed successfully as separate tasks. It is this schism between the different imperatives that sees emergency and

\footnotetext{
11 “...coordination was a major challenge after the earthquakes in Afghanistan (1998), Turkey (1999), Gujarat (2001), and Bam (2003). In most cases this was due to the involvement of large numbers of humanitarian organizations and multiple levels of government" (ALNAP, 2005; Hicks \& Pappas, 2006).

12 "...immediately after the disaster, numerous national and local nongovernment organizations (NGOs) and over 100 international organizations, such as the United Nations, international nongovernment organizations (INGOs), European Union, North Atlantic Treaty Organizations (NATO), bilateral partners, etc., arrived in the earthquake zone to aid the relief effort." Hicks \& Pappas (2006) on the response to the 2005 Kashmir earthquake.
} 
temporary shelter provided for victims followed by a delay in construction of permanent shelter, forcing the emergency solutions into semi-permanent ones. "The fear is that any focus on development, that is, reconstruction, could potentially divert already constricted funding from humanitarian assistance programs." (Hicks \& Pappas, 2006). Developmental and humanitarian concerns are one in the same - both are aiming to restore liveable conditions to affected peoples, both focuses are essential to successful reconstruction, and neither can do this successfully without the other.

The issue is that while the humanitarian work done by these organisations saves lives and provides the necessities of life in a time when they can be scarce, their forays into building permanent houses have often failed. The danger is that just like the delivery of healthcare and emergency shelter, these large groups can view housing in the same way - as an end product, rather than a process (Wahlroos, 1999; Baradan, 2006). There are many examples of post-disaster complexes built from this attitude that have simple failed, a picture painted vividly by Barakat (2003):

“Opportunities to enhance post-disaster recovery efforts or introduce mitigation measures are usually overlooked, and little or no distinction is made between the provision of physical shelters and the provision of homes. Lack of experience leads to assessments that do not provide the relevant information, and projects that are impractical and appropriate neither to what beneficiaries need, nor to what they want. As a result, reconstruction projects are often unsustainable: at best, houses are remodelled by their occupants; at worst, they are simply rejected and abandoned."

The motivation for NGOs creating permanent housing solutions as a product may stem from the way that they operate financially. Heavy reliance is on donations from benefactors which are used to fund rescue and relief operations; in return the benefactors want tangible evidence and accountability from the NGO. This means that to continue funding levels, and to find new sources, the organization 
must be able to quantify their efforts ${ }^{13}$ so physical contributions like houses are preferable to material or management assistance in reconstruction. The problem is that these houses may not be suitable. Box housing in Dücze City, Turkey after the 1999 earthquakes (Baradan, 2006); Concrete dome houses near Jogjakarta, Indonesia; Relocated villages after the 2001 Gujarat earthquake (Barenstein, 2006). These are all examples of reconstruction projects that have been unsuccessful because they do not meet basic living requirements of the inhabitants. The Gujarat example is particularly relevant - the reconstruction of permanent houses occurred through several different methods and the success of each has been recorded by post-occupancy surveys. Done by Barenstein (2006), the survey covered 21 villages in the affected area and included five different models of housing reconstruction, with the average percentage user satisfaction shown in parenthesis:

1. Owner driven reconstruction (ODR) with technical support from NGO's (94.5\%)

2. ODR with subsidisation from the government (Cash approach)

3. Community driven reconstruction (CDR) with support from a local NGO (90.8\%)

4. Contractor driven reconstruction, in situ

5. Contractor driven reconstruction, relocated

(Barenstein, 2006; Jha, et. al., 2010)

\footnotetext{
${ }^{13}$ Illustrated particularly well by the IRFC Operational Updates - documents published by the Red Cross outlining exactly where and how their resources have been spent and what this has achieved. (IRFC, 2007)
} 


\begin{tabular}{|c|c|c|c|c|c|c|c|c|}
\hline \multirow{2}{*}{$\begin{array}{l}\text { Reconstruction } \\
\text { approach }\end{array}$} & \multirow{2}{*}{$\begin{array}{l}\text { Degree of } \\
\text { household } \\
\text { control }\end{array}$} & \multicolumn{2}{|c|}{ Form of assistance } & \multicolumn{3}{|c|}{ Role of actors } & \multicolumn{2}{|c|}{ Location } \\
\hline & & Financial & Technical & Community & Agency & Contractor & In-situ & $\begin{array}{l}\text { New } \\
\text { site }\end{array}$ \\
\hline Cash Approach & Very high & Cash only & None & None & None & $\begin{array}{l}\text { Household may } \\
\text { hire }\end{array}$ & Yes & No \\
\hline $\begin{array}{l}\text { Owner-Driven } \\
\text { Reconstruction }\end{array}$ & High & $\begin{array}{l}\text { Conditional } \\
\text { cash transfer } \\
\text { to household }\end{array}$ & $\begin{array}{l}\text { TA/Training of } \\
\text { household }\end{array}$ & None & $\begin{array}{l}\text { Project } \\
\text { oversight } \\
\text { and training }\end{array}$ & $\begin{array}{l}\text { Household may } \\
\text { hire }\end{array}$ & Yes & No \\
\hline $\begin{array}{l}\text { Community-Driven } \\
\text { Reconstruction }\end{array}$ & Medium to high & $\begin{array}{l}\text { Transfer to } \\
\text { household or } \\
\text { community }\end{array}$ & $\begin{array}{l}\text { TA/Training } \\
\text { of community } \\
\text { and household }\end{array}$ & $\begin{array}{l}\text { Project } \\
\text { organization } \\
\text { and oversight }\end{array}$ & $\begin{array}{l}\text { Project } \\
\text { oversight } \\
\text { and training }\end{array}$ & $\begin{array}{l}\text { Community } \\
\text { may hire }\end{array}$ & Yes & No \\
\hline $\begin{array}{l}\text { Agency-Driven } \\
\text { Reconstruction } \\
\text { in-Situ }\end{array}$ & Low to medium & $\begin{array}{l}\text { Funds handled } \\
\text { by agency }\end{array}$ & $\begin{array}{l}\text { Limited or } \\
\text { none }\end{array}$ & Limited & $\begin{array}{l}\text { Management } \\
\text { of project }\end{array}$ & Agency hires & Yes & No \\
\hline $\begin{array}{l}\text { Agency-Driven } \\
\text { Reconstruction in } \\
\text { Relocated Site }\end{array}$ & Low & $\begin{array}{l}\text { Funds handled } \\
\text { by agency }\end{array}$ & $\begin{array}{l}\text { Limited or } \\
\text { none }\end{array}$ & Limited & $\begin{array}{l}\text { Management } \\
\text { of project }\end{array}$ & Agency hires & No & Yes \\
\hline
\end{tabular}

Figure 14 Approaches to reconstruction in Gujarat (Jha et al, 2010)

This shows a direct relationship between the participation of the affected and the satisfaction at the quality of housing. The survey showed that $100 \%$ of those in houses made from the first approach reported conditions as being better than before the disaster, while many of the houses from the fifth approach ended up abandoned due to insufficient construction quality and materials, "...no privacy for women, and there was no space for cattle, fodder, agricultural implements and people's furniture ... a high percentage of households explicitly said that their new home had no positive features at all." (Barenstein, 2006)

The growing consensus is that the contractor driven or top down, approach is largely an unsustainable one and that the success of projects can often be directly related to the involvement of the local population and incorporating cultural sensitivity into the solution (Barakat, 2003; Bektaş, 2006; Diacon, 1997; Khan \& Moore, 1990). It is this involvement which then becomes a driver for planning any post-disaster reconstruction projects, “...outside help should seek to facilitate this process by making sure that building materials and skills are locally available at affordable prices, or free of charge" (Barakat, 2003). The role of these NGO's is a crucial one, and the issue for this research is how the humanitarian concerns of these organisations can assist the developmental concerns of the people, the communities and the nation. Can the temporary 
shelters be seen as more than just temporary, can they be the base for the inhabitants to construct a permanent house?

\section{The Role of the Affected}

As mentioned previously in this chapter, projects and research advocate the engagement of the population and community as a key component in reconstruction. It is perhaps summarised in a direct way through the attitude of a resident of an affected community, Anil Mukim, in Kutch two months after the 2001 Gujarat Earthquake:

"Now I tell you that this relief thing should come to an end. We should get out of this relief mentality. We should encourage more and more people to stand up and start working and begin rehabilitating themselves. Then, I can assure you, if you come here after one year, you will not be able to make out whether an earthquake had ever hit this place." (Malik, 2001)

This embodies the ability and the desire of communities to be self-sufficient, to be able to be in control of their reconstruction process. It also shows a backlash against the 'relief mentality' and, beneath this, what has been raised as an issue previously in this chapter: the overwhelming aid response, the gifting and the handouts. When displaced people have to wait on someone else to build their house for them, delays can cause extreme frustration. The role that the affected can play in reconstruction is invaluable and can be summarised in three points: their physical input, their cultural input, and the benefits this has for them. 
First, the affected communities are a source of labour. It is an inescapable reality that the economics of reconstruction is a primary concern. With potentially thousands of people displaced in need of shelter, the cost of each reconstruction becomes vital in order to make use of what funding is available. By enlisting the displaced themselves in providing the labour for rebuilding it can largely eliminate the labour costs, which can be up to half of the total, that come with agency driven projects. It may then be that, as was the case in the Core Shelter Project covered in the next chapter, many houses can be made under the supervision of a single trained foreman. "In the aftermath of an emergency, peasants could all manage to rebuild their own homes faster than they could do if they have to wait for an agency to build them for them." (Kakumbli, 2004)

Second, it is a valuable source of information on norms and expectations. Involving community groups into the planning process will give a frame of the expectations that a community may have, as well as essential components and spaces that must be present in the reconstruction. Some cultures may require an open, well ventilated area for cooking; others will need strong partitions within to support basic

"The primary resource that can be tapped for post-disaster reconstruction is the grassroots motivation of the affected individuals, their friends, and families. Assisting groups can help, but they must avoid duplicating, replacing, or stifling any action best taken by the affected population themselves. Emphasis should therefore be given to supporting and maintaining the dignity and self-reliance of the disaster affected population. Before providing outside support, it is vital for assisting groups to check whether locally available expertise, labour, and products are available within the communities. It is preferable to use these resources rather than import skills and materials from outside. This helps strengthen local capacity for reconstruction."

(EPC, 2004) privacy. The lack of any of the basic cultural requirements of a people will mean that even their most basic of daily routines are not supported, and so the house will be unsustainable as a long-term solution. Reconstruction should be predicated on active, two way participation between beneficiary communities' and reconstruction agencies/authorities at all levels. (Barakat, 2003)

Finally, this is a psychological issue. 
The displacement and destruction wrought by a major disaster can be an extremely stressful time for those who have lost so much. Highlighted in Halvorson and Hamilton's (2009) interviews with survivors in the months after the 2005 Kashmir earthquake is the sense of distress and loss:

“'Money can be made, buildings can be rebuilt, but I can't get my family members back'. Another respondent commented, 'I lost everything- there is nothing left'. Other significant impacts that were mentioned included the loss of peace of mind [and] mental anguish."

Involving the displaced people in the reconstruction process provides a focus during this time of loss and hardship; instead of waiting, in different shelters and temporary dwellings, on agencies to arrive and provide permanent housing. The benefit is not just a meaningful contribution towards their home. It is also being able to prove ability and skill sets which will enable them to alter and renovate their house in the future, as life gradually returns to a more normal state and more resources become available. Finally, it is being able to give a greater sense of ownership and pride in their new dwelling. The loss of something as personal and great value as a home is devastating. It is for this reason that gifted homes on borrowed land rarely work - there is no sense of place or ownership. It may be a shelter, but equally it may not be a home.

It is finding an effective way to involve all levels of assistance effectively and one of the key components of this is having a plan in place before the disaster strikes so that when the times comes, the roles and responsibilities of the difference parties are clear. "After the analysis [of the Turkish Earthquakes], we can conclude that the most important period in the post-disaster housing reconstruction is the pre-disaster period" (Baradan, 2006). An exclusion of government at any level could see land issues arise or create an unwanted distance for the new houses from regional or national assistance in the future, since they may be perceived as belonging to some other group or organisation. National government can also provide invaluable funding towards materials for reconstruction projects, and the same can be said for NGO's. Both of these groups also have the capacity to assist in expert construction knowledge and 
techniques and, with some oversight of projects, can ensure that valuable mitigation techniques are used in construction ensuring the structures have capacity to resist future disaster events. But as well as this, the benefactors must be a part of the process, "community participation has to be an integral and foundational component of a project's design, not simply a politically correct, cosmetic add-on" (Barakat, 2003). 


\section{Chapter 4 - Case Study and Construction}

\section{Introduction}

This chapter will expand on what has been covered in the previous chapters by looking at case studies of post-disaster reconstruction projects. The first, the Core Shelter project in the Philippines, highlights findings from the last chapter. It shows how successful a 'bottom-up' approach can be and what the roles and relationships between the government, agencies and the recipients themselves were to achieve this. It also shows how simple mitigation techniques can be employed to resist future events. The second case study is an example of a topdown, agency driven project that initially failed to meet the needs of the inhabitants. By looking at post-occupancy assessments, it is an example of how the inhabitants have altered the houses so that they meet their spatial and cultural needs, and the consequences of these alterations. It will also outline the role that materiality plays in the reconstruction process and the importance of taking into account the culture of a population.

\section{Core Shelter: Typhoon resistant housing in the Philippines}

The Core Shelter project in the Philippines has been successfully reconstructing houses after typhoons and other disasters for many years now. The Philippines archipelago is one of the most disaster prone areas in the world, being hit by landslides, earthquakes and volcanic eruptions. The most prevalent is typhoons, with around twenty major ones entering the region annually; between eight to ten (PRNC, 1994) of these making landfall and causing widespread damage (Diacon, 1997). The Core Shelter project began in the late 1980's as a response to the destruction of around 207,000 houses by Typoon Sisang in 1987 and by 1992 it had produced over 16,000 dwellings (Diacon, 1997). The project still continues today and has been successful for several reasons: the core house is resistant to 
future storm events, it is cheap to construct, and it involves local skill and materials and is relatively fast to set up. The objectives and aims of the project, described by Diacon (1992) are as follows:

1. To assist in the rehabilitation of disaster victims

2. To reduce the number of families rendered homeless every year by providing a structurally strong core shelter made of indigenous materials that can withstand 180 $\mathrm{km} / \mathrm{h}$ winds

3. To develop and promote self reliance among the beneficiaries and the community; and

4. To maximise the participation and engage the commitment of beneficiaries and the neighbourhood in the construction of structurally strong houses

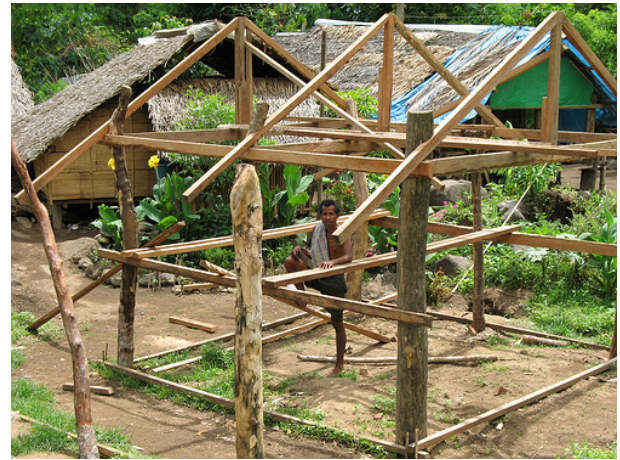

Figure 15 Frame of a core shelter

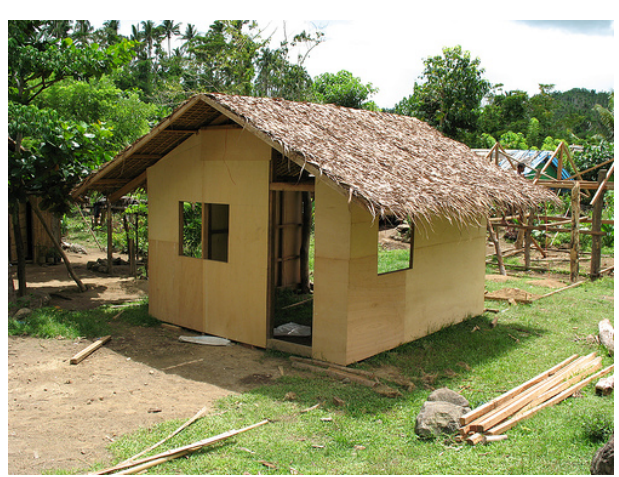

Figure 16 Core shelter with thatch roof

Of particular note is why the project is referred to as a 'core-shelter'. The idea is that the houses provided aren't just simple houses but are constructed so that they will remain standing in future storm events. This means that the destruction and economic loss of typhoons is less and the value of the houses is greater, since they don't have to be rebuilt after major typhoons. The core shelter design was initially tested after Typhoon Sisang by building an initial 450 units. After all shelters withstood two typhoons the following year, with wind speeds of 160 and $175 \mathrm{~km} / \mathrm{h}$, the scheme was expanded (Diacon, 1992). The design is simple; indeed it needs to be so it can be constructed locally:

"The core shelter technical design incorporates anchorage tying the roof to the ground through cement footings to achieve continuity, and a four sided roof 
design strengthened by roof trusses. An essential aspect of the design is that it is easy to understand and build. The technology is thus acceptable to local people who can be trained in the simple methods required." (Diacon, 1992)

The shelters are constructed so as to make use of locally sourced materials and local labour. Consequently the cost of the shelters are low; the cost of one shelter in 1992 being 13,500 [NZ\$417] pesos each (Diacon, 1992), the cost almost 15 years later being 10,000 pesos [NZ\$308] (NCIP, 2006) and the majority of these costs being materials. "Since the wall and roofing material does not have any strong bearing on wind resistance, strong indigenous materials are used to reduce costs" (Diacon, 1992).

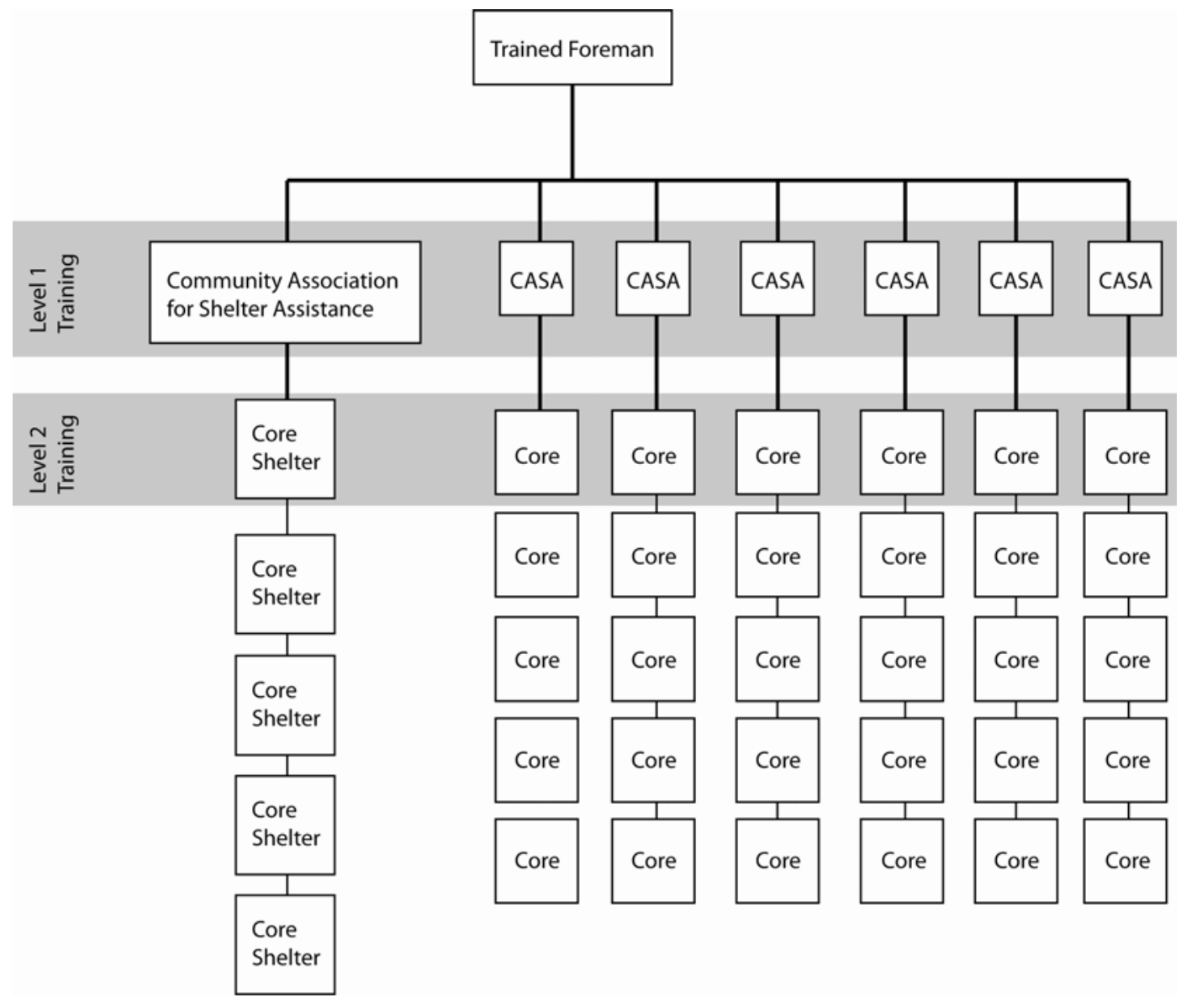

Figure 17 User participation and Training Model.

The construction of these core dwellings is driven primarily by the affected people and community groups. At the outset of a new project, beneficiaries are organised into groups called Community Associations for Shelter Assistance 
(CASA), consisting of five families each. These CASA groups are put under the guidance of one trained foreman and go on to receive two levels of training. Level 1 is a day and a half course based on the theoretical aspects, ensuring understanding of not just how the houses are built, but why they are constructed in such a way. Level 2 is the construction of the first house for one of the families, taking three days and demonstrating the technical aspects of the construction. The other units for the CASA are then built by the families, with only very limited supervision. Each foreman is generally responsible for around 50 core shelters, or 10 CASA's; this bottom up method means than many shelters can be built simultaneously and "has proved a highly workable and beneficial system of operation' (Diacon, 1992).

Finally, the project allows a 'modular approach to construction'. The shelter provided is necessarily small, measuring around $3.0 \times 3.5 \mathrm{~m}$, but it is given with the intention of the inhabitants upgrading it 'at a later date according to their resources and needs' (Diacon, 1992). "Families typically build extensions around this core to increase the size of their accommodation, since average family size is 8 or 9 persons" (Diacon, 1997). The shelter that is deployed is very basic (Module A) but should the recipient have more resources available at the time of construction $^{14}$, other options are available:

- Module B consists of upgrading and improvement through the provision of Cement Hollow Block walls and concrete slabs for the main floor and toilet.

- Module C consists of upgrading through the provision of door and window panels and interior finishing

- Module D consists of upgrading through the provision of galvanised iron corrugated roofing sheets, gutters, downpipes, etc. (Diacon, 1992)

\footnotetext{
${ }^{14}$ Funding for the basic core shelter comes from the beneficiary, local government, the Department of Social Welfare and Development (national government), and NGO's.
} 


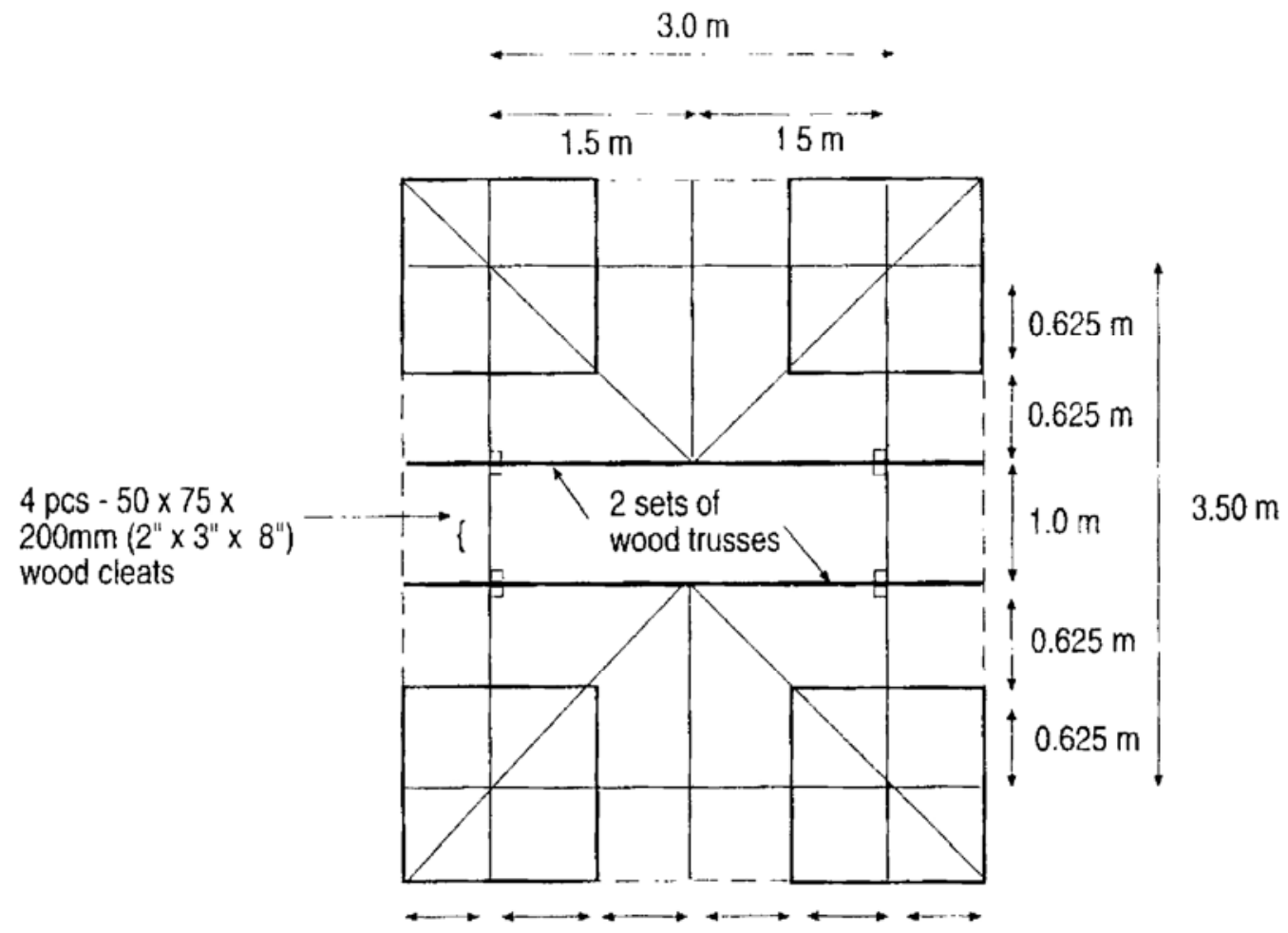

$0.5 \mathrm{~m} 0.75 \mathrm{~m} 0.75 \mathrm{~m} 0.75 \mathrm{~m} 0.75 \mathrm{~m} 0.5 \mathrm{~m}$

Figure 18 Roof plan for housing core. (Diacon, 1997)

The lessons from the core shelter project can be distilled from the factors that made it a success, some of which also reflect findings and background from previous chapters. The inclusion of the end users is an important part of a successful process. Involving them, indeed relying on them, in the primary stage of construction not only gives a stronger sense of ownership and occupation; the inclusion also provides a platform. A way to prove to themselves that they have the skills necessary to build and add on to their house, to give them a focus and a confidence during a time marked by loss. The use of materials is a contributor to this. Using materials that have a presence in the area can avoid any confusion of feeling of alienation or difference that may arise from using foreign materials. It is the bottom-up approach, the cheapness of construction and the resistance to future collapse; all of these in combination make this an effective solution and it is where some of these components are missing where projects can begin to fail. 


\section{Modification of a Product: Düzce City, Turkey}

On 17 August 1999, a 7.6 magnitude earthquake struck northwest Turkey (known as the Marmara earthquake) leaving approximately half a million homeless across the area. The nearby city of Düzce was among the locations badly damaged and was subsequently hit by another earthquake (Düzce earthquake) of 7.2 magnitude less than three months later in November 1999. The destruction caused a widespread response, both on the national and international levels; the two earthquakes cause an estimated 300,000 houses to collapse (Arslan \& Unul, 2006). The city was gradually reconstructed through the establishment of 15 temporary settlements and permanent housing complexes which:

"On one hand ... met the urgent needs of families affected by disaster (i.e. shelters, sense of secure, safety, privacy and daily life requirements). On other hand, they hindered the maximization of the opportunity given by the disaster itself to be turned into sustainable re-development due to their negative impacts in a long-term." (Bektaş, 2006)

Three years after the disaster, Bektaş undertook an occupancy evaluation of some of the temporary settlements that were still being used in order to determine the positive and negative aspects of them and identify how, if at all, they have been altered by the occupants to meet their needs. The study of how the units were changed and altered to meet the spatial and cultural requirements of the inhabitants highlights several important points. It gives an idea of how people adapt to houses that are unsuitable for their requirements. It also highlights the need for taking into account cultural requirements in the reconstruction process. 


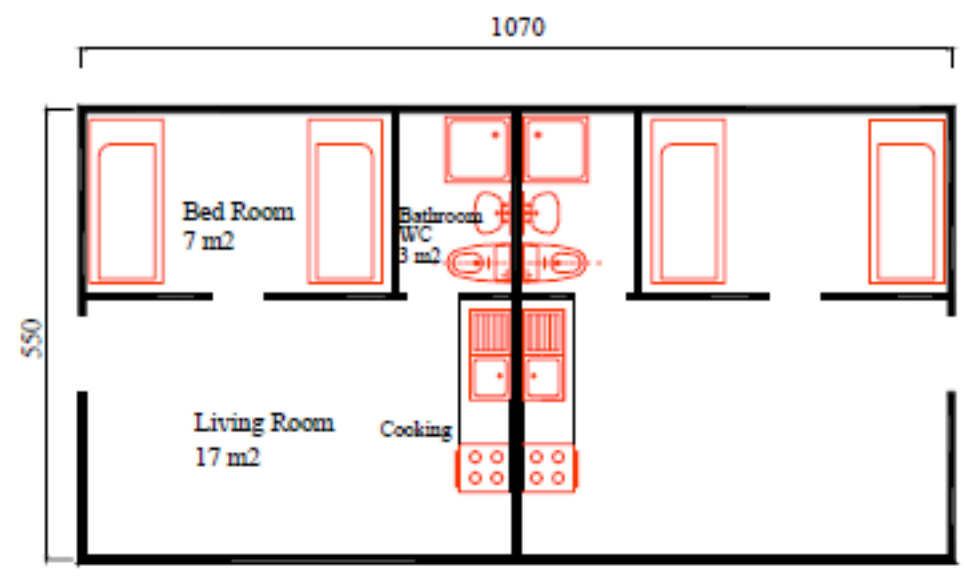

Figure 19 Initial design of temporary housing unit. (Bektaş, 2006)

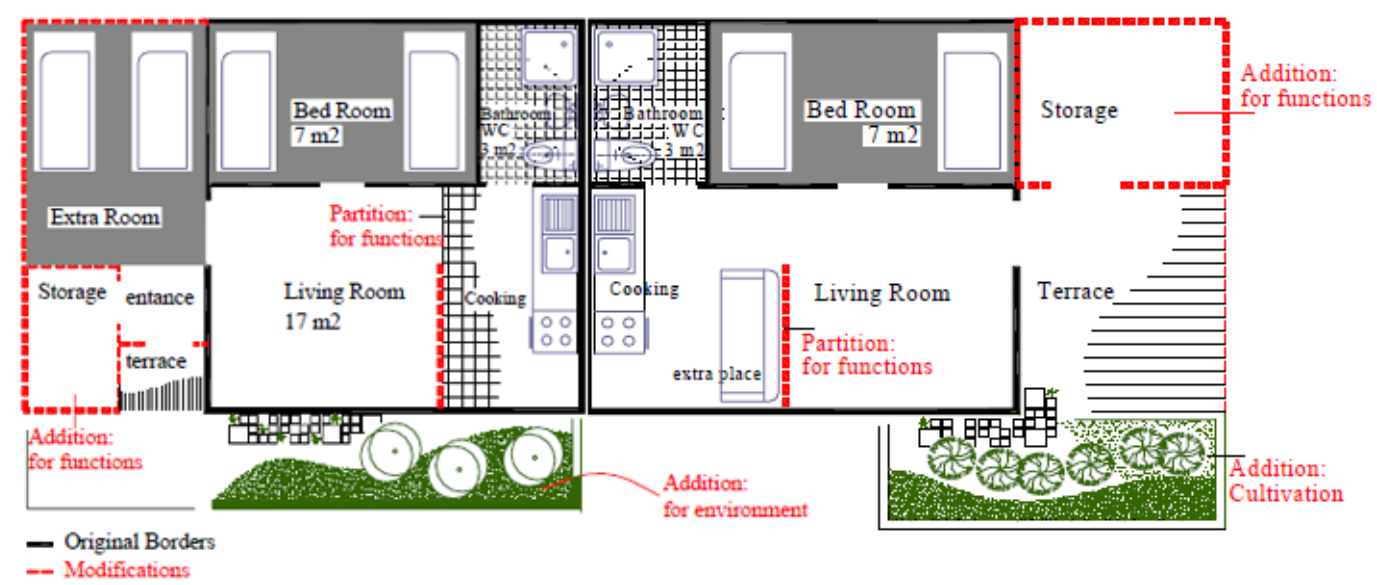

Figure 20 Units modified by residents. (Bektaş, 2006)

Shown in Figure 19 and Figure 20 are the differences between the original construction and a typically modified house. These were layouts built for the Fevzi settlement and was selected by Bektaş for study because of the relatively high level of user satisfaction, due to "...the stage of privacy with multi-roomed character and size of the units" (Bektaş, 2006).The living room has been further partitioned to allow separation and privacy between the living area and kitchen, as well as due to ventilation issues and fire risk. The other frequent modifications noted are the addition of extra bedrooms if family size requires, kitchenette, storage and greenery, or terrace space. The green space and wind breaks added 
by the inhabitants are to provide a transition space between the private and public, to give more privacy from the neighbours; a semi-private buffer zone (Bektaş, 2006).

The overall theme of these modifications is towards greater privacy. The initial houses had some division of space but because both the amount of privacy and the space provided were insufficient, the house was further modified. This case also becomes a counterpoint to the Gujarat example given in the previous chapter, where the contractor built houses were eventually abandoned. The Düzce case is another of agency driven reconstruction, in itself a common mode of failure, that has not met the basic cultural needs of the inhabitants. However instead of being abandoned, they were heavily modified to meet the spatial needs. "The typical resident attitude observed is to modify housing to tackle with this problem by using own capabilities." (Bektaş, 2006)

This modifying made the houses more habitable, but they were still far from comfortable. The construction of the houses was not suitable in the long term for the climate of the region; "Particular feature of imported products is that they are not considered being resident against extreme climate conditions in disaster affected region as high humidity or dryness" (Bektaş, 2006). The combination of cheap construction techniques employed by the contractors and the lack of knowledge in construction of the inhabitants when making alterations created issues. Because the houses were perceived as just temporary solutions, the materials used were low quality and unsuitable for the area, “... [The] high humidity caused early rusting of the steel framework ... wooden products become unusable because these required protections not counted in reconstruction phase against extreme temperature changes." The solution proposed at the time (2006) was for the military to come and demolish the entire settlements. (Bektaş, 2006). While the residents did modify the designs, this too ended in failure due to the low quality of them, "...these drudgeries were done unconsciously due to lack of know-how." (Bektaş, 2006). 


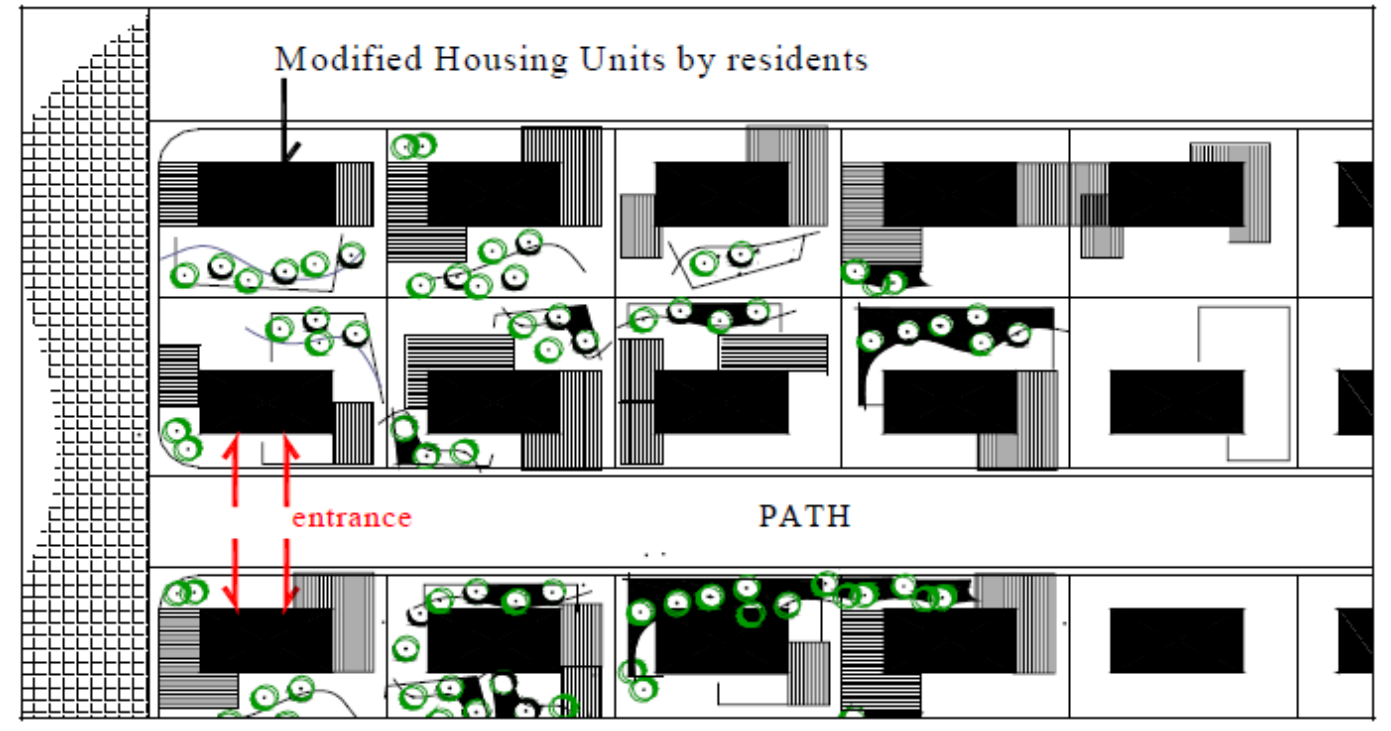

Figure 21 Schematic of modified settlement, Fevzi Cakmak settlement. (Bektaş, 2006)

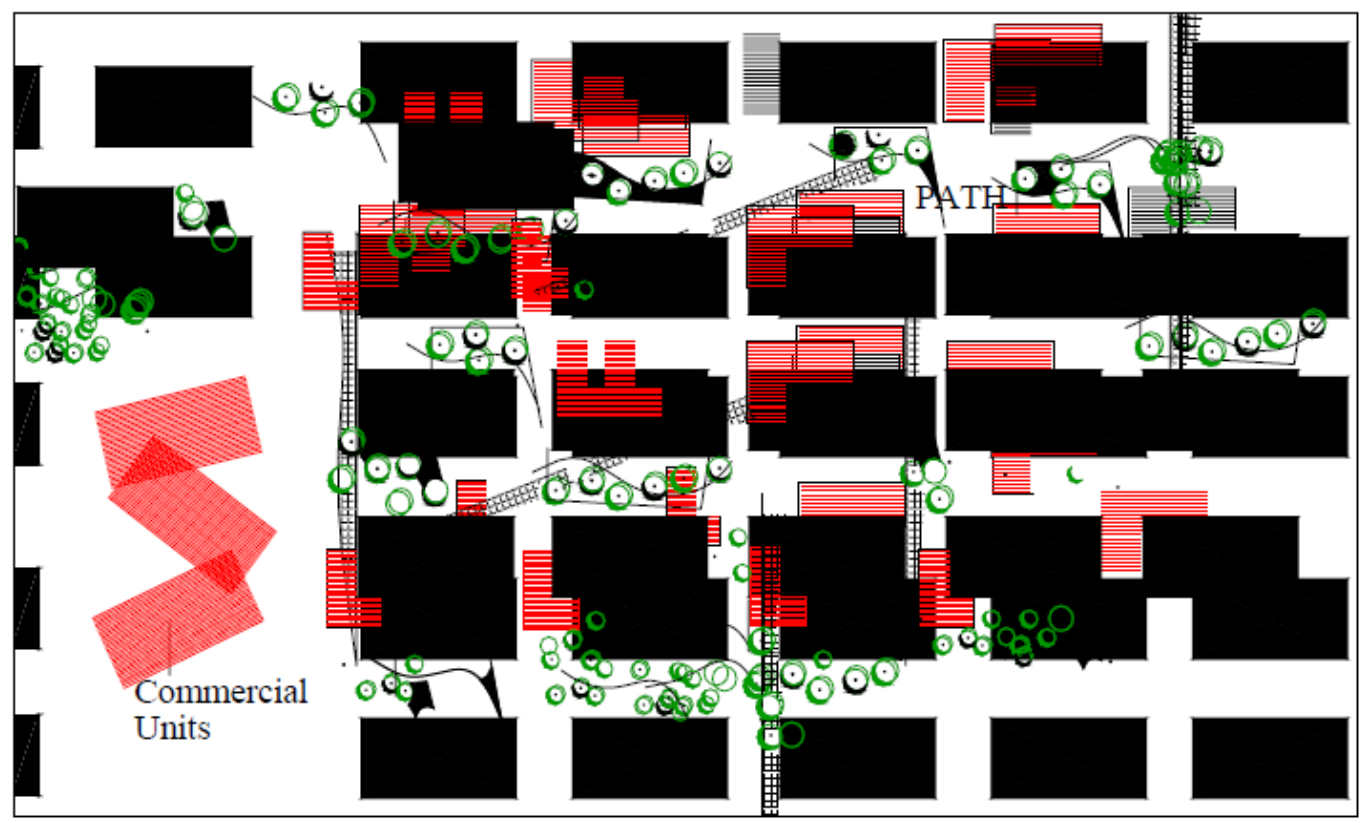

Figure 22 Schematic drawing to express modifications and spontaneously built commercial units in Fidanlik settlement. (Bektaş, 2006)

As well as the example from this case study of user driven modifications, it also highlights one of the main issues driving this research - that temporary housing isn't always very temporary. When shelters are designed with an expiry date, we get what has happened in Düzce - inadequate housing used long term that can lead to eviction and displacement again, compounding on the uncertainty and 
instability that has already occurred after the disaster. "Due to the delay of the reconstruction stage, in some cases the rehabilitation [temporary housing] stage may continue for up to 30 years" (Acerer, 1999 cited in Çelebioğlu \& Limoncu, 2006), "In such cases, the temporary houses undertake extemporary functions related with usage style and period." (Çelebioğlu \& Limoncu, 2006).

\section{The Role of Materiality}

The role that materiality has to play in reconstruction has been touched on in the previous chapter. In order for reconstruction to aid the wider community, it should be locally sourced or manufactured to aid the rebuilding of the local economy. It should be familiar so that the affected population can easily use it in reconstruction. In addition to this, the choice of materials and construction systems should aim not just to provide a shelter but to, as in the case of the Core Shelter Project, mitigate against future disaster events. Material selection can also impact quality and comfort for better, where it aids climatic or privacy requirements, or for worse, as in the Düzce case where poor construction lead to insufficient thermal quality.

Designing and constructing to resist future events first means understanding what disasters are likely to strike the area, and what reasonable steps can be taken in construction to resist them. "Opportunities to enhance post-disaster recovery efforts or introduce mitigation measures are usually overlooked, and little or no distinction is made between the provision of physical shelters and the provision of homes" says Barakat (2003), citing the confusion following a disaster as a reason why this is often neglected. These measures will differ depending on what an area is vulnerable to. For earthquakes and cyclones, structural mitigation is being able to resist the large lateral loads that these disasters create. By bracing vertical structural elements laterally or by ensuring that connections between elements (between roof and walls, for example) are sufficient to transfer loads, buildings may then resist the next disaster. For floods, mitigation is building on land located above vulnerable low lying areas or, if this is unavoidable, raising the house on piles so it is above flood levels. For 
other disasters such as volcanic eruptions or tsunamis, no amount of structural strength can resist the forces that are created so mitigation is limited to being located away from vulnerable areas, as was done in Sri Lanka after the 2004 Indian Ocean tsunami. It is often the lack of these considerations that cause so much damage in earthquake scenarios; Charleson (2008) that vulnerable buildings in developing countries stem from "...weak of non-existent regulatory environments, a lack of enforcement of design and construction standards, and a lack of technical and professional leadership." Charleson \& Fyfe (2001) identify 'configuration irregularities' as a common source of structural failure and suggests simple solutions to create more resistant structures; regular column orientation and regular plan shape. Many of these recommendations also apply to cyclones; regular plans resist wind better and ensuring the roof, walls and foundations are connected and secured sufficiently can reduce the risk of failure (Agarwal, 2007).

The selection of materials contributes to a buildings structural resistance. Selecting materials that provide sufficient ductility to transfer forces will ensure a greater chance of withstanding the next disaster. This can be as simple as making sure materials like brick and concrete are reinforced, or the frames in a timber house are connected well and braced.

Material choice is also driven by the prevailing climate in an area. The priorities of what comfort a house must provide varies greatly and material choice must reflect that. Tropical hot regions require sun protection without restricting ventilation; monsoon regions require a high level of water tightness, often in addition to shade and ventilation; arid desert regions need thermal barriers against the constantly fluctuating temperatures, and colder regions need to be able to contain heat. Often vernacular material choices or techniques reflect these climatic, as well as cultural needs. Success in reconstruction then may come down to combining properties of vernacular materials, or the materials themselves, with mitigation techniques to give sufficient resistance. "All cultures have developed adequate and affordable housing solutions; if these are used as a starting-point, appropriate housing would be easier and cheaper to provide" (Wahlroos, 1999). 


\section{Culture, Context and its Relation to Reconstruction}

A population's traditions and beliefs have an indelible impact on how they live their daily lives. From daily routines to privacy to leisure, these traditions have shaped the relationship that they have to their house. Rapoport (1984) outlines the definition of culture with three aspects:

1. Culture as a way of life typical of a group, a particular way of doing things

2. As container of symbols, meanings and cognitive schemata transmitted through symbolic order

3. As a set of adaptive strategies for survival related to the ecological setting and its resources. (Rapoport, 1984)

Essentially these are the routines, beliefs and setting of a population. Relating this back to Maslow's (1943) Hierarchy of Needs from chapter 2, it is the 'survival strategies' that take greatest importance; ensuring that any shelter or house provides basic protection from the 'ecological setting'. This is followed by supporting the inhabitants 'particular way of doing things', which relates directly to Quarantelli's (1995) main distinction of a post disaster house as opposed to a shelter - that it supports the resumption of daily household activities and routine. Finally, it describes culture as a container of symbols and the house is one of these.

What has happened in past reconstruction cases is that those in charge of rebuilding are not part of the affected population. As discussed in the previous chapter, this has been the cause for failure and abandonment of the project through ignorance of local culture. However, another issue arises when western or 'developed' ideas of the house are imposed over top of how a community has traditionally functioned. Jigyasu (2004) describes the reconstruction of Marathwada and Gujarat after their respective earthquakes as examples of this;

“...during post disaster rehabilitation, the decision-makers perceived provision of 'modern' and 'city like' layout and housing for the villages, as benchmark for 
their development. The negative consequences of these in the long run are evident in the Marathwada case. Also in these areas, provision of reconstructed houses is thought of as an end product for development of villagers."

Khan and Moore (1989) write that there are two 'streams of consciousness' in models for housing: the modern and the traditional. Describing the modern ideal as "...uncompromisingly western ... of advanced technologies, the good life" it can be seen as the antithesis of the traditional; the ubiquitous concrete apartment blocks, the suburban detached housing that has become so prevalent. The traditional then is interpreted as the vernacular, that which has been developed around and shaped by the context in which it exists. Khan and Moore (1989) write of the interior of a vernacular house as "...decoration which speaks of 'identity' and the craft of tradition, of place and timelessness", echoing Rapaport's (1984) definition of a container of symbols. The last century, especially the last few decades, has seen information and lifestyles of how other societies live and operate much more readily available; an increase in global awareness. This awareness may mean that, as people can easily compare their lifestyles to others, the notion of 'the good life' of western society will become increasingly desirable. Future developments taking place in this constantly changing world, not exclusively reconstruction projects, will have to strike a delicate and intangible balance between new technologies and cultural and vernacular traditions.

This begins to provide a framework to developing a solution in post-disaster housing; there is not just a personal aspect of needs, but a cultural one. Reconstruction is inextricably tied to this and the other ideas that have been discussed in this paper. Quarantelli's (1995) phases of post-disaster reconstruction reflect the priorities of rescue, recovery and the humanitarian aspects that are so important after a disaster. It and other models highlight the reasons for a staged reconstruction - the scarcity of resources, human and civic priorities. Maslow's (1943) hierarchy presents the needs, from physiological to social, of the individual and Rapoport (1984), Khan and Moore (1989) provide a cultural framework to relate the individual's needs to their surroundings. These are the aspects of post-disaster reconstruction that respond to a disaster and a 
location. So, in order to design a solution, specific disasters and locations must be used to identify the parameters that these may imply, and to serve as a test for a proposed design solution. The next chapters will address the design component of this thesis by identifying sites and parameters, and outlining how a solution may be developed to address these issues. 


\section{Chapter 5 - Site and Context}

\section{Introduction}

The challenge for developing a solution that can provide post-disaster shelter and housing is the broad nature of the disasters themselves and where they strike. Although the scope of disasters has been narrowed significantly for this research, it still presents a huge proportion of the world that is subject to these events. It covers the majority of countries in the world; areas that aren't affected, like large parts of western Africa or Europe, are subject to natural disasters outside this scope. It covers a wide range of climatic conditions, from tropical rainforest to arid desert to temperate and continental climates. It represents a huge diversity in cultural and social practices, ethnicities and religions. It also, perhaps most significantly, covers a wide range of building technologies, materials, methods and styles.

In order to undertake some level of meaningful testing of solutions, it is necessary to take a cross-section of this wide variety of conditions that exist. Factors that have informed the choice of sites are as follows:

- $\quad$ Disaster frequency: sites have a history of being affected by disasters, as well as a perceived future vulnerability. This may be that the climate exposes them to seasonal weather patterns in the case of cyclones or floods, or their location is one of seismic weakness for earthquakes or volcanoes.

- Disaster intensity: the site is not just exposed to disasters, but is prone to large events that may cause total collapse of some buildings; this necessitates the reconstruction process.

- $\quad$ Poverty indicators (GDP, median income etc):

- $\quad$ Climatic conditions (temperature, rainfall, wind)

- $\quad$ Prevalent materials

- $\quad$ Typical housing forms (foundation, roof, plan types)

- $\quad$ Prominent religions

- $\quad$ Basic housing functions 
While it would be ideal to take a large selection of sites across the globe to represent these conditions accurately, time constraints necessitate only a few sites be selected to ensure that these can be studied in appropriate depth to be able to develop an effective solution. Four sites have been selected for investigation of the research and for design development, representing four broad geographic areas:

- $\quad$ Iriga City, Philippines

(East Asia/ Pacific)

- $\quad$ Bhuj, India

(South Asia)

- $\quad$ Ankara, Turkey

(Middle East/Europe)

- $\quad$ Rio de Janeiro, Brazil

(Latin America)

\section{Site Selection}

The first catalyst for any site is that they must be prone to natural disaster(s). As identified earlier in chapter 2 , this means not only that large natural events occur, but that they happen in such a location as to cause severe damage to both the physical and social aspects of a society or community, that is not only affecting buildings and people but also disrupting routines, structure and basic functions of a society. So the sites are located where natural events occur (see Figure 50 to Figure 53).

The sites are also regions exhibiting significant populations of poorer people. "A natural disaster hotspots study... identifies 47 countries in which more than 50 percent of the population is at relatively high mortality risk from two or more natural hazards. Of these countries, only three are developed: Japan, the Republic of Korea, and Taiwan (China)" (Amin Goldstein, 2005). It is these poorer nations, where the average income is low, which suffer greater economic impacts (Figure 23). Because there is less money available to aid in the rehabilitation, the impact on the affected can be more severe and long lasting. The images below illustrate this. Figure 23 shows disaster loss as a monetary cost; richer nations lose more, but viewed as a percentage of GDP it is the poor 
nations who are worse off. Figure 24 shows the human cost of disasters - global deaths by income groups, with the lower class representing the vast majority.

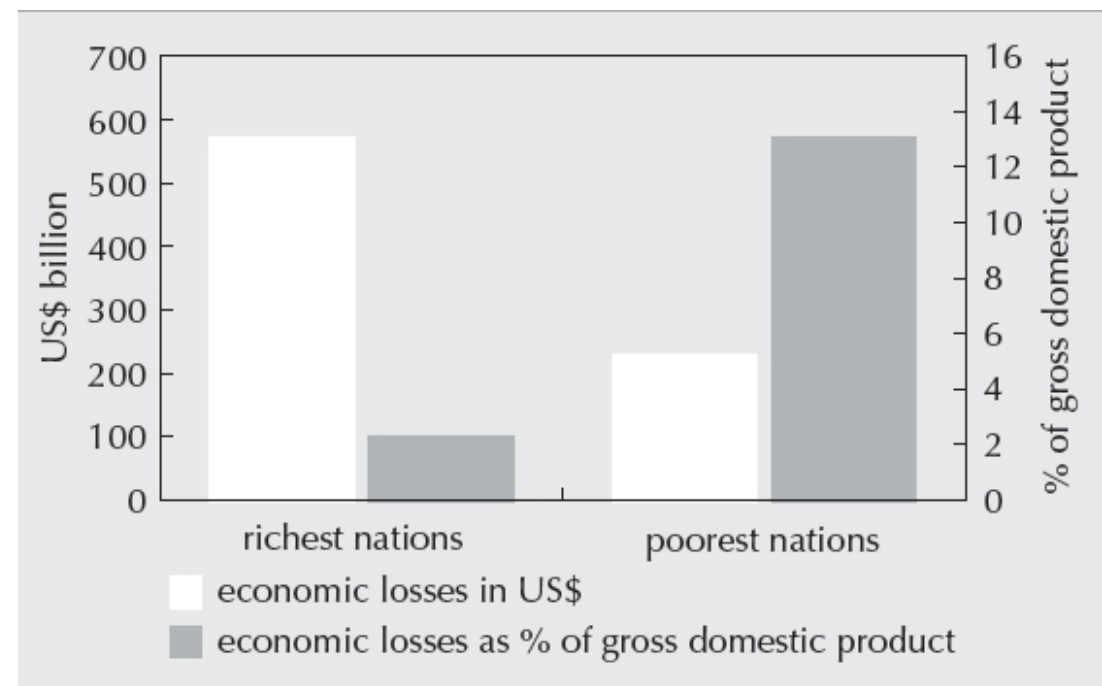

Figure 23 Economic loss: actual cost and as \% of GDP (Amin \& Goldstein, 2005

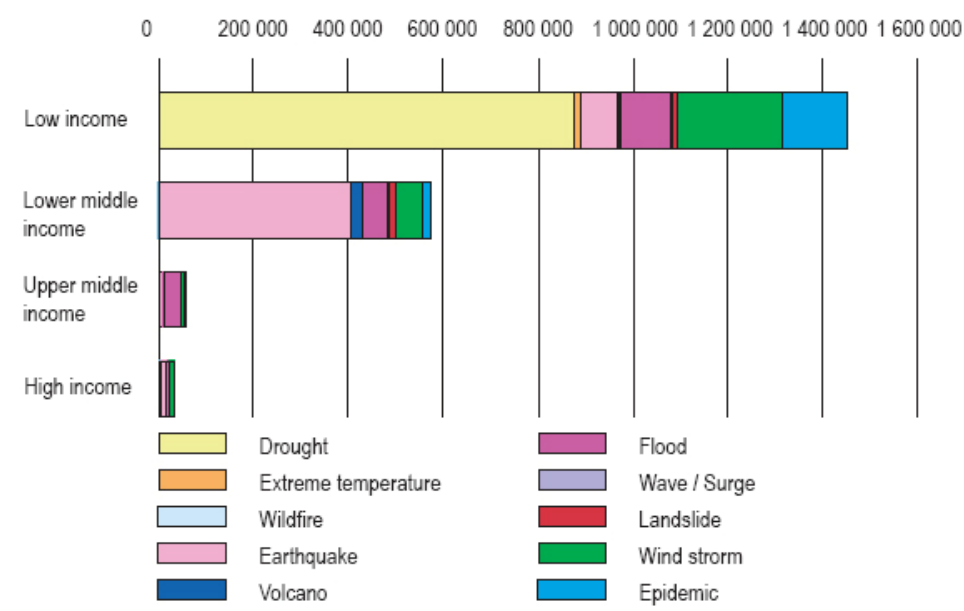

Source: EM-DAT: The OFDA/CRED International Disaster Database - www.em-dat net - Université Catholique de Louvain - Brussels - Belgium, 2004, based on elaboration by ADRC

Figure 24 Number of people killed (by income class/disaster type), World summary 1973 - 2002 (UN, 2004)

Sites were also chosen to represent a range of natural disasters. Each disaster has different impacts on the built environment and on the population in terms of injury and life loss. Most importantly for this research, they are an event that must be considered structurally in reconstruction. Houses in areas prone to earthquakes or cyclones must be able to resist stronger lateral forces than 
houses in other areas. In areas prone to flooding, houses may be raised above inundation levels or must be built on raised or sloped ground.

\begin{tabular}{|l|l|}
\hline Site & Disasters Experienced \\
\hline Iriga & Earthquakes \\
& $\begin{array}{l}\text { Flooding } \\
\text { Cyclones } \\
\text { Volcanoes }\end{array}$ \\
\hline Bhuj & $\begin{array}{l}\text { Earthquakes } \\
\text { Flooding } \\
\text { Cyclones }\end{array}$ \\
\hline Ankara & Earthquakes \\
\hline Rio de Janeiro & Flooding \\
& Landslides \\
\hline
\end{tabular}

Table 1 Disaster vulnerability at selected sites

The sites were also chosen to represent a range of climatic conditions. Their climates are recorded here using the Köppen-Geiger climate classification system which divides the earth's climate into five broad categories - tropical, arid, temperate, cold or polar - and then finer subcategories within these

- Ankara: Continental Mediterranean climate (Csb); close to Cold Semi-arid climate (BSk) and Warm Summer Continental climate (Dsb)

- Bhuj: Hot Desert climate (BWh)

- Iriga: Tropical Rainforest climate (Af) close to Tropical Monsoon (Am)

- Rio de Janeiro: Tropical Savannah climate (Aw) close to Humid Subtropical climate (Cwa) 

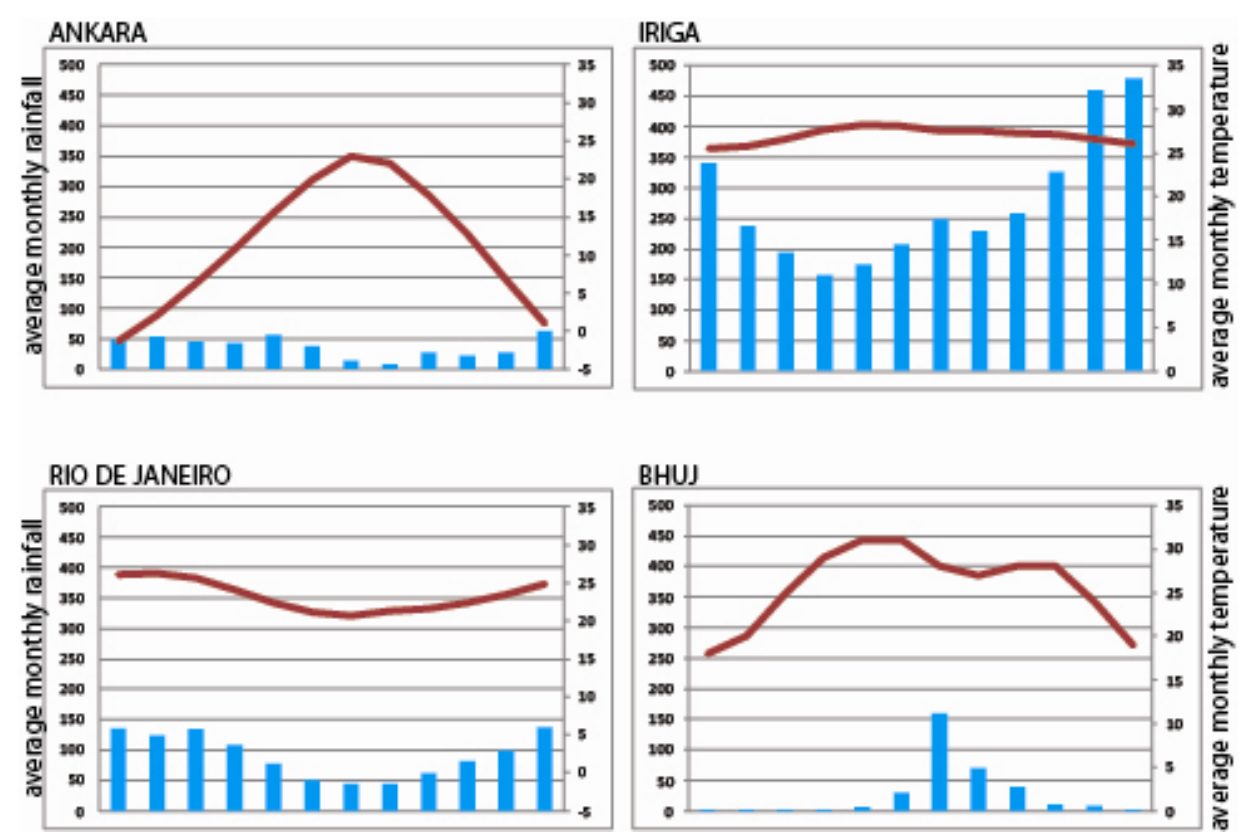

Figure 25 Average temperature and rainfall of selected sites

The differences in climates present four very different conditions. Tropical Iriga with very high rainfall all year round and constant temperatures to arid Ankara with a seasonal climate producing warm brief summers and cold winters; they all present challenges and conditions to take into account in planning for reconstruction.

Housing typologies and vernacular styles were also considered in selecting sites. This broad subject encompasses many different variables; of particular consideration for reconstruction were floor plan arrangements, spatial relationships and levels of privacy, cooking methods and the materiality of vernacular styles.

Sassu (2007) identifies traditional housing plans as being rectangular, circular or linear; noting that the type of plan present is usually 'related to many cultural, historical, and urban planning traditions'. Circular plans are predominant in nomadic cultures across the world, used by the nomadic herders of the Gobi desert to the Maasai of southern Africa. These circular type dwellings are less prevalent in permanent settlements, seen most commonly in North Africa and found more scarcely as isolated examples or historic community styles (Oliver, 2007). The most widespread form, in various incarnations, is rectilinear plans; 
varying from a simple square or rectangle, to a courtyard style to a long, linear house. It is this style that informs the base of a solution. This rectilinear form, while not as efficient as the circular, is still very efficient in resisting the lateral forces that come from earthquakes and high winds providing it is properly braced.
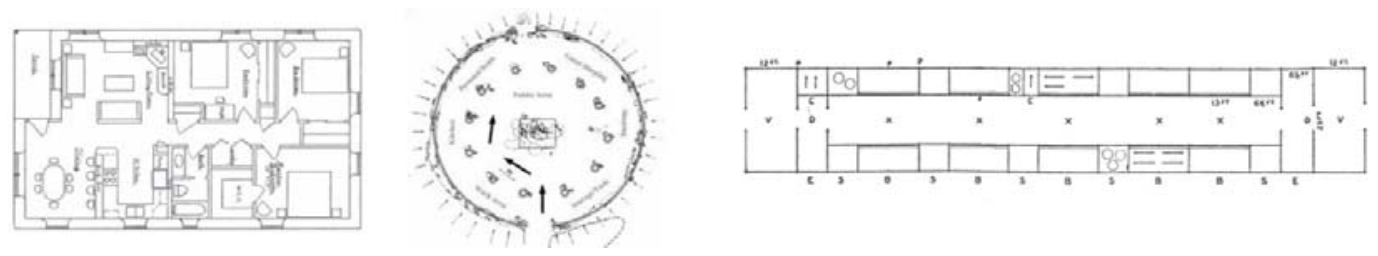

Figure 26 Rectangular, circular and linear type plans

The role of local materials and knowledge of these has been discussed in the previous chapter and so the sites have been chosen to exhibit different building traditions and resources. This is partly a product of climate, as this and geography dictate the natural resources that have been available. Regions with higher or more consistent rainfall tend to have more vegetation, and be able to re-grow faster, than drier or seasonal regions and so have traditionally used timber and grasses as a primary building material. In contrast, arid regions have traditionally used other materials such as mud, stone or earth in various incarnations. This is not to suggest that a reconstruction must use traditional materials, rather it is an indication of familiarity and acceptance of a particular construction style. Indeed, with globalization now many of these traditions have been lost and are no longer relevant to cultures that formerly relied on them; reinforced concrete may be a more appropriate option than mud brick or adobe. The material used to construct permanent houses will be dependent on availability, affordability, lifespan and suitability to the climate; all of which is dependent on the location of the reconstruction. 
Prevalent Materials

$\begin{array}{ll}\frac{\text { F }}{\text { Form moulded sun dried brick }} \\ \frac{\text { Fired brick in west }}{\mathrm{C}} & \text { Timber framing }\end{array}$

Palm for thatching and walling

믄 Timber framing

Bamboo roof/frame/wall/floor

Fired brick

Corrugated iron

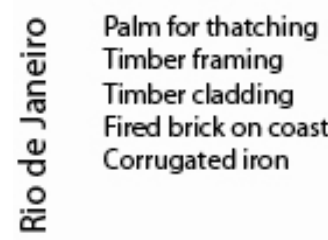

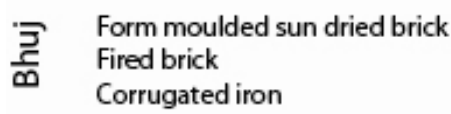
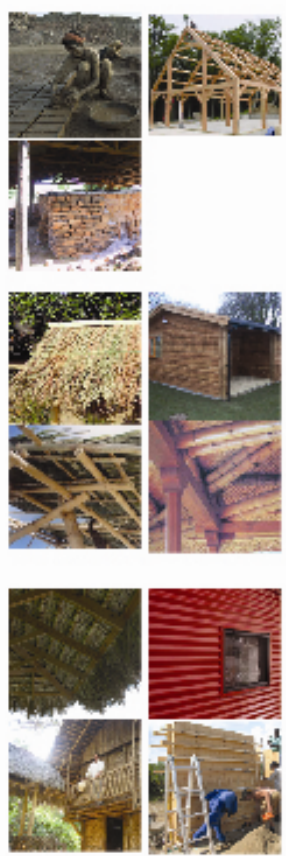

Clay tile roofing

Palm leaf thatch roofing

Bamboo matting curtain wall

Clay tile roofing

Palm leaf + grass thatch roofing
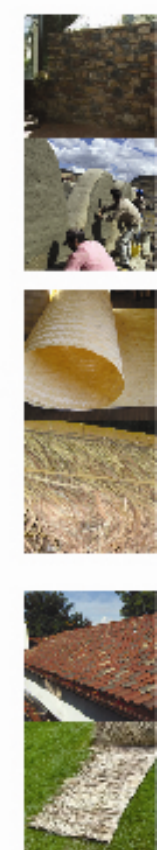

Clay tile roofing

Grass + straw thatch roofing
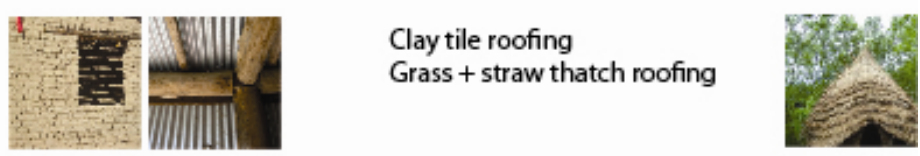

Figure 27 Regional styles (Oliver, 2007)

The consideration of how spaces and functions of even the most basic dwelling can be vital to a project success. Particularly the provision of cooking space is important. Not simply providing one, but understanding how they are used. Whether open (or outdoors) or semi-open space is required or if cooking is done using a hearth or an enclosed fireplace; expected privacy levels can be a major requirement for some cultures. As seen in the Düzce example in the previous chapter, a lack of basic privacy can cause a house that is not suitable for even basic living. Two of the sites, Ankara and Bhuj, have significant Muslim populations (99\% and $21 \%$ ), who require especially high levels of privacy both from outside and within the family unit. Significant cultural factors like these will have an impact on how a space or spaces can operate. 


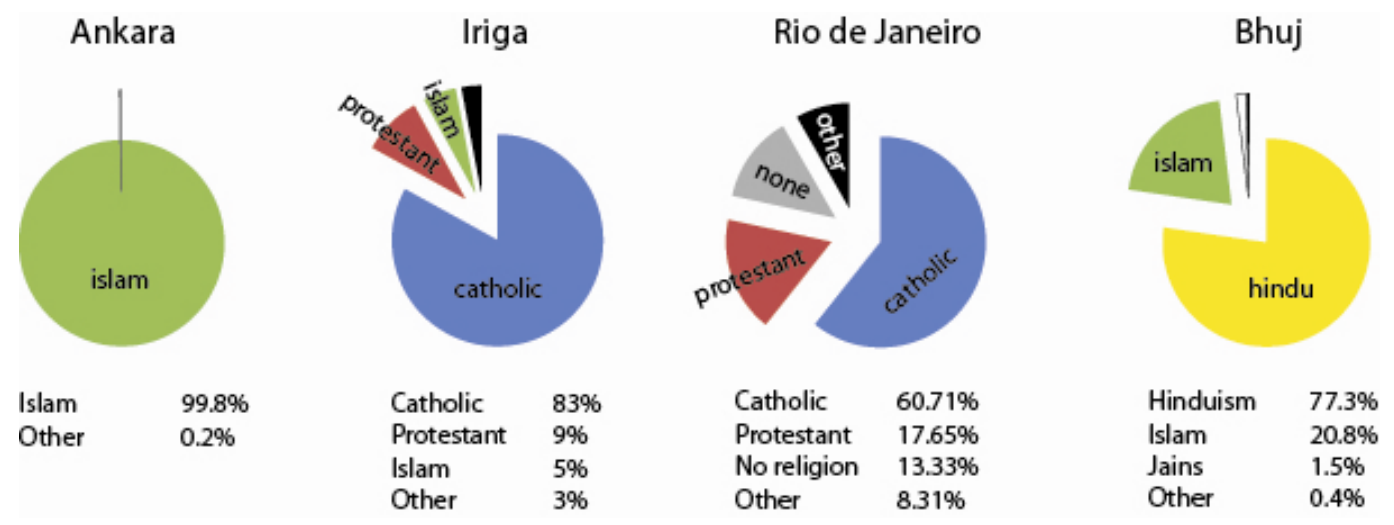

Figure 28 Religious demographics of selected cities

Finally the sites represent different urban contexts. While no exact site within these locations is to be identified, each of the sites has different characteristics that would influence reconstruction there (see Figure 29 to Figure 32). There is a distinct split between the denser, urban sites of Ankara and Rio de Janeiro and the less populated regions of Bhuj and Iriga evidenced by the satellite photos. The space available for a house is potentially much less in these areas; many houses in Ankara traditionally used two or more stories to make the most use of the land, while the largely informal favelas of Rio de Janeiro are restricted to small shack-like dwellings perched on the steep hillsides on "...supports, such as stilts, on a rock bed" which enables them to withstand the rains (Oliver, 1997). Iriga and Bhuj do not have the same density as the other sites, so can be afforded more land. Indeed a tradition of courtyard housing exists in the subcontinent, mixing indoor and both covered and open outdoor spaces to create private but well ventilated spaces. 


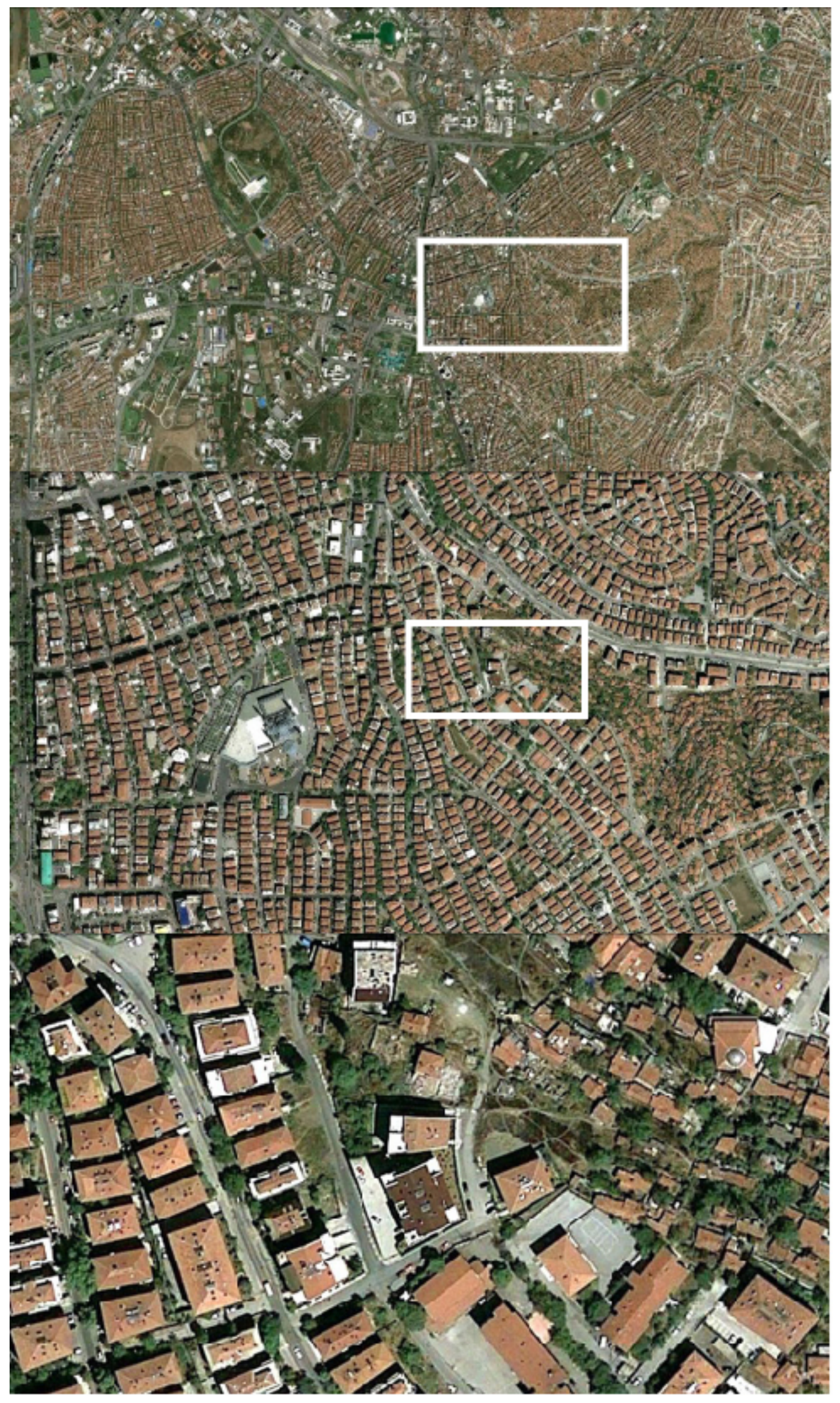

Figure 29 Satellite photos of Ankara, Turkey 


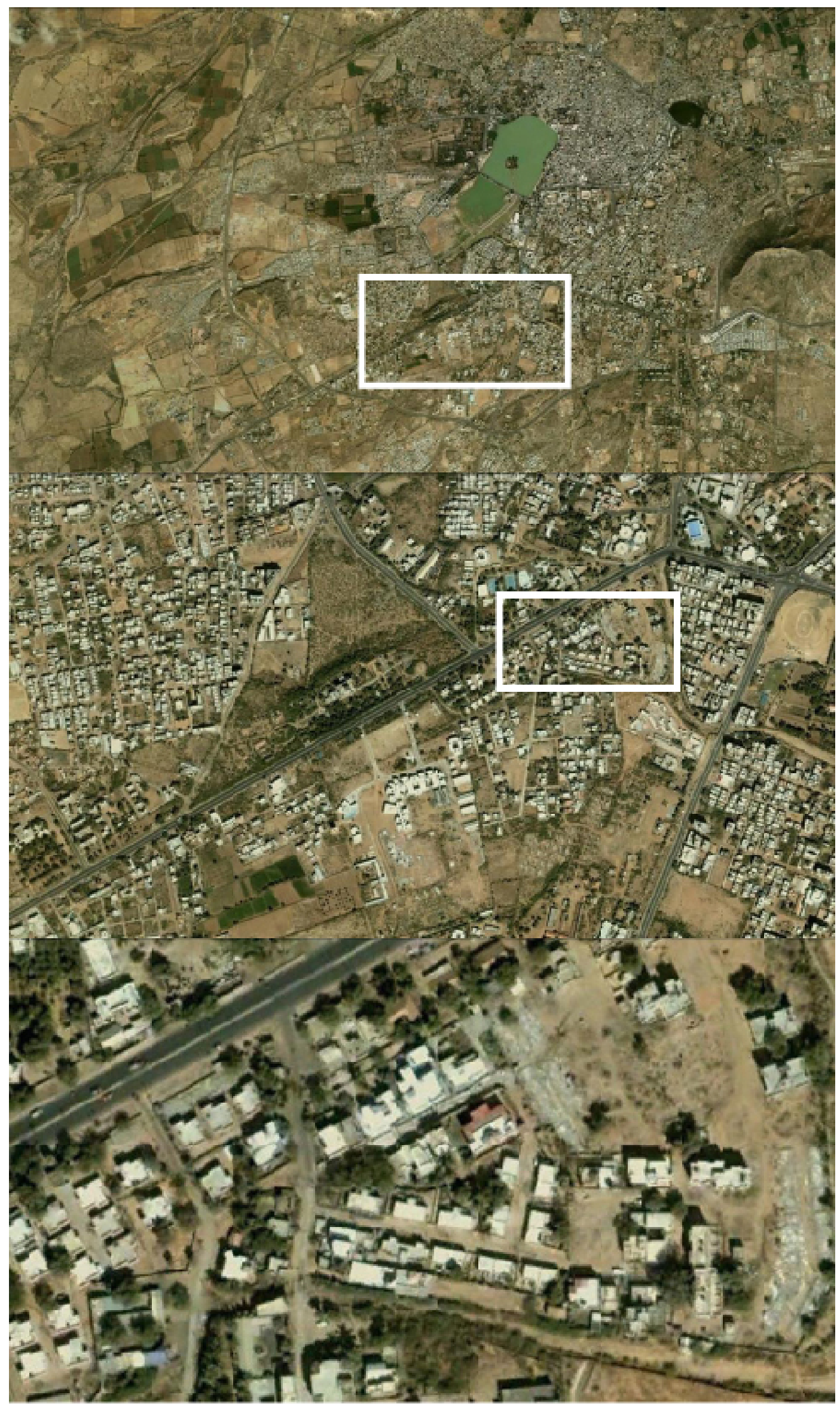

Figure 30 Satellite photos of Bhuj, India 


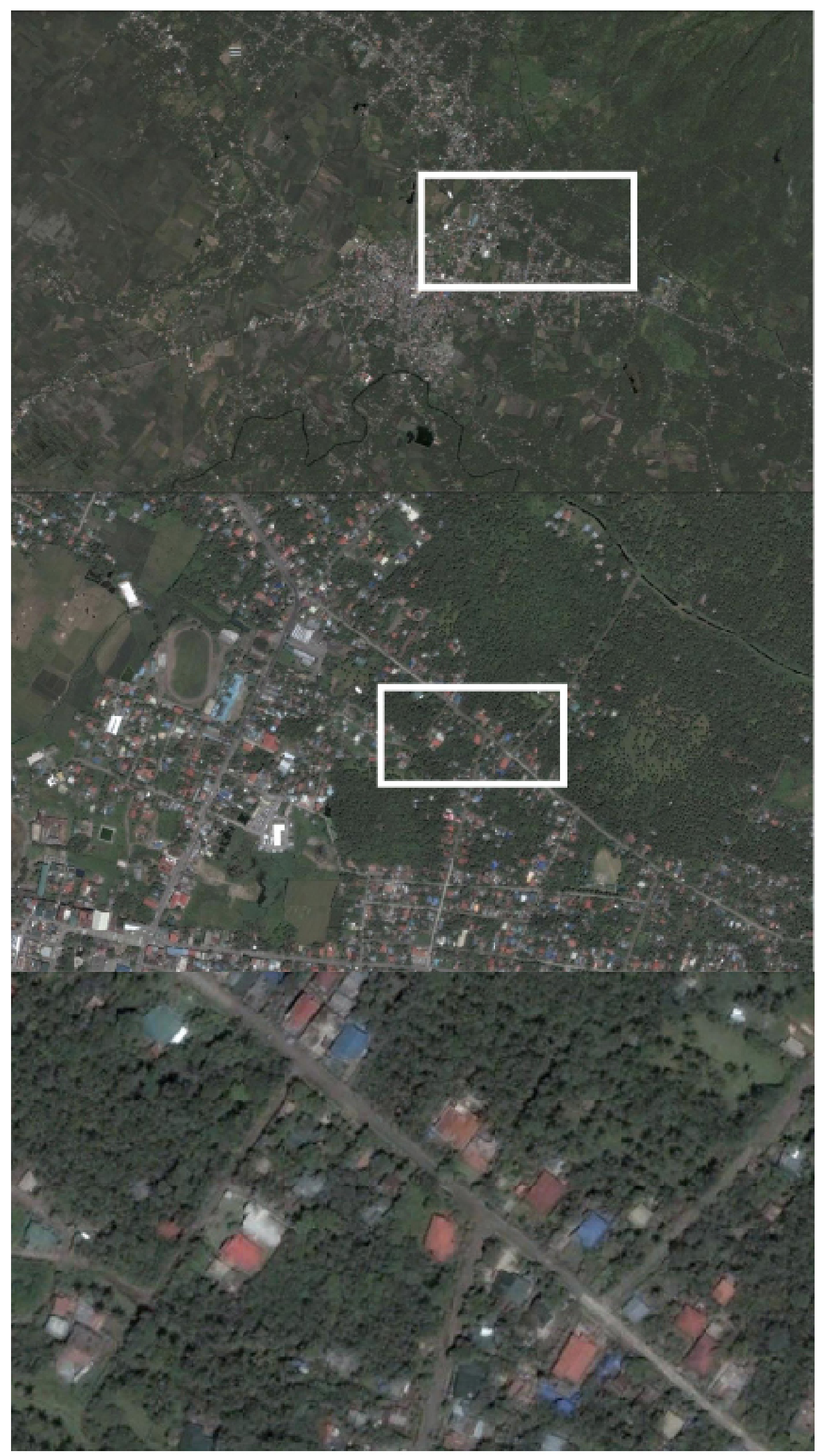

Figure 31 Satellite photos of Iriga, Philippines 


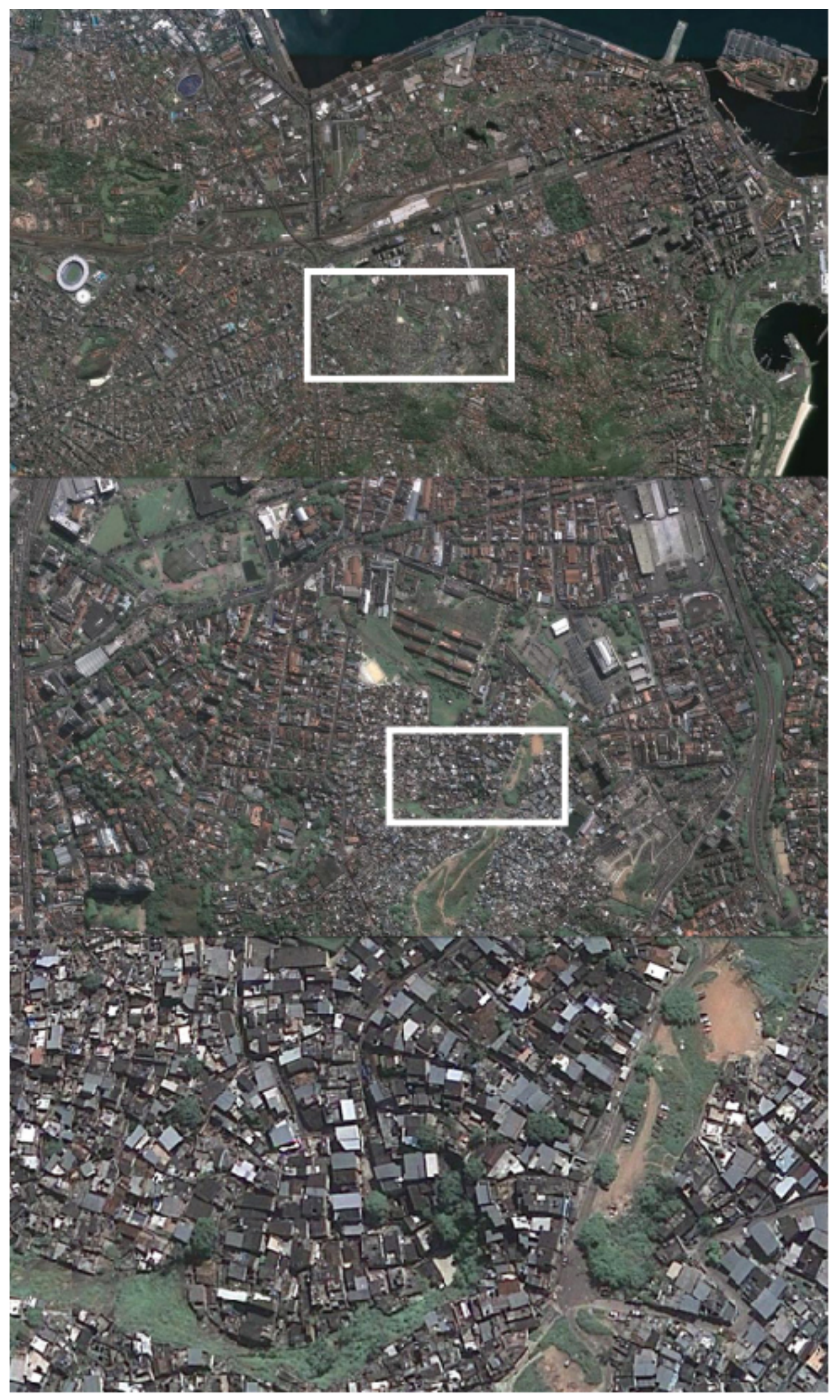

Figure 32 Satellite photos of Rio de Janeiro, Brazil 


\section{Chapter 6 - Design}

\section{Introduction}

The aim and motivation of the design component of this research is to create a built solution that can bridge the temporary and permanent stages of the reconstruction process. The stage models that have been discussed in chapter 2 differ depending on who has proposed them and what their primary focus is. What is taken from this is that, regardless of how the stages are defined, there is a need to rebuild in steps. Those who have been displaced require a shelter immediately and waiting for a permanent house to be constructed may not be feasible, especially due to resources being put into rescue and humanitarian services in the weeks following the event. This is why temporary and emergency shelters are useful; it is when these temporary shelters continue to be used beyond their intent that is the core issue of this research.

The design is focused on developing a temporary shelter deployment that will form the base, or core, of a future permanent house. This is guided by the needs of the affected as defined by the lower three levels of Maslow's hierarchy psychological, safety and love and belonging or, in reconstruction terms; shelter, routine and resource security, and social relationships, including the wider community. The stages of the design will also serve to provide defined roles for the groups that may become involved in the reconstruction process: the different levels of government, the NGO's, the community and the beneficiaries.

\section{Concept}

The proposed stages of the designed solution consist of three parts:

1. The initial deployment of a shelter,

2. Using the shelter to construct a small permanent house and finally,

3. Additions to this core house to meet the long term needs of the inhabitants. 
Quarantelli's $(1989,1995)$ post-disaster reconstruction model, discussed in chapter 2, outlined four phases - three variations of temporary shelter and a final permanent house. It must be remembered that this phase model is not a proposal for a solution, or a preferred way of approaching a reconstruction scenario; it is a depiction of the phenomena that existed, and in some cases still does exist, in the case studies observed for the research. The attitude that underpins reconstruction in such a style is still one that sees housing as a product. The progression of the temporary shelters and houses, each one more suitable than the last one, reads much like the concept of a waiting room at a clinic - making people minimally comfortable until the service that they need can be provided.

Comparatively, the proposed stage development for this design consists of one temporary shelter and two or more stages of permanent housing. The emergency shelter stage that Quarantelli identifies may still exist in the proposed model, since it is a very temporary solution measured in days; the nature of it often being mass accommodation in civic structures, it is a different solution to family unit sized shelters and so is not considered to be part of the designed solution presented here.

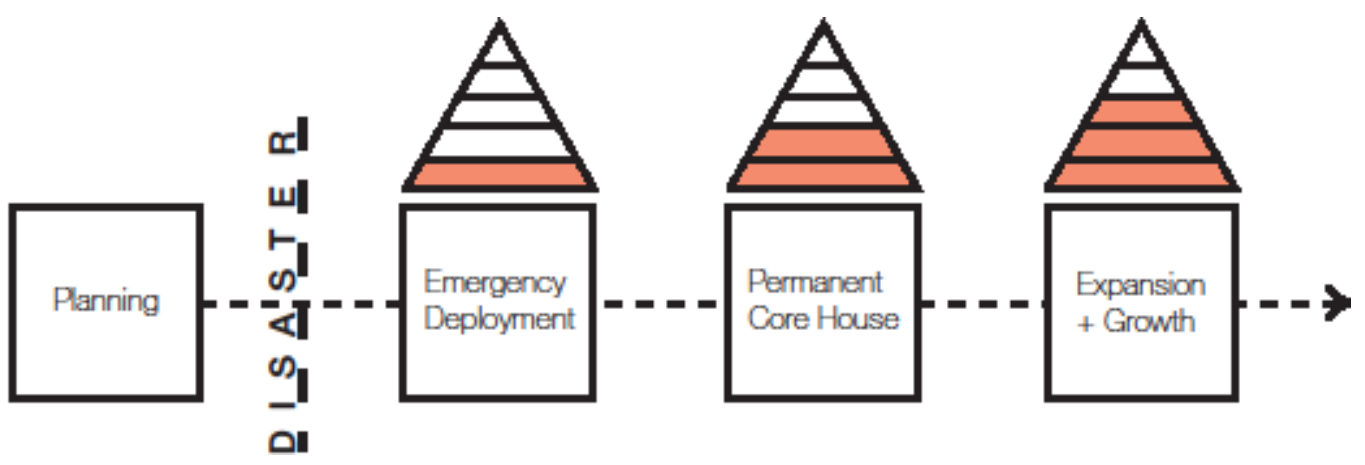

Figure 33 Stage development of the proposed design

There are several ideas which drive this solution; the primary one being a direct response to the research problem of preventing slowed redevelopment, the issue of people having to use temporary solutions as permanent ones. Driving the designed component is the idea that none of the stages experienced will be 
purely temporary. The specific uses of components may change from one stage to another, but the inhabitants can make changes and adaptations knowing that they will not be relocated to a new dwelling.

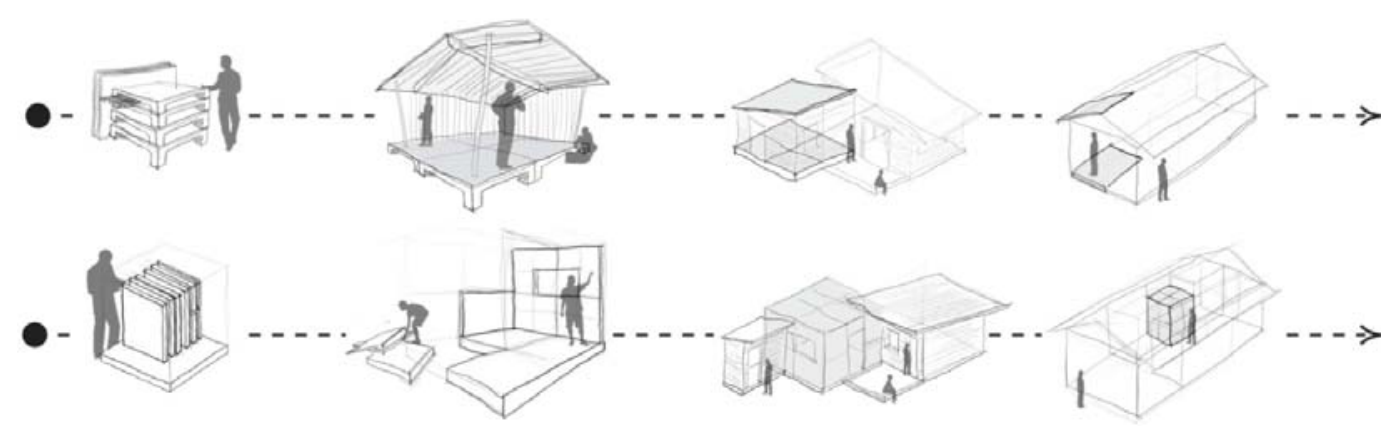

Figure 34 Early concept sketches of deployment, shelter, core and expansion phases.

Creating a solution that is determined in part by the beneficiaries and the community has informed aspects of the design as well. The failure of previous projects due to unsuitability to the cultural needs of the people or providing housing that is completely alien in form or materials, as well as how some of the successful projects have been conducted indicates the need for a construction approach that integrates local elements. This includes involving the beneficiaries directly in the rebuilding stages as labour and supporting local industry and economy by sourcing or creating materials locally, but it is also proposed that it will extend beyond this into the early planning stages. Community involvement in the planning stages will inform how the housing solutions will develop: information gained from consultation can determine what the most crucial aspects of a house are; what spaces or elements must be part of the core and what can take shape in the following stage; what levels of separation and privacy are needed, both internally and to the public. How existing houses respond to these needs can inform this, but inclusion of the communities gives them a voice in their reconstruction. 


\section{$\underline{\text { Process }}$}

An emergency or temporary shelter in post-disaster scenarios is currently widely used in cases of mass displacement. The key difference that this design seeks to achieve in the initial deployment is that it provides a temporary shelter, but that the components of it are to become permanent parts of the future house. The integration of the deployment installs it with a greater value to the users; the temporary stage is not useless once the shelter phase has ended, it is integrated and becomes a permanent fixture.

The concept of the deployment is two parts: a base, or platform, and a roof or cover. It seeks to achieve the basic necessities of housing - shelter from the climate and a degree of privacy. At this early stage it is little more. It is unlikely that any kind of utilities or services will be available so, as is often the case in emergency phases, ablutions, water supply, electricity and other services are not part of the shelter; the response at this point is focused on saving lives and preventing further injury and risk. In order for the emergency shelter to be able to be dispatched rapidly, it is proposed that they are manufactured locally (either at a regional or national level). This is also important to ensure, through creating and planning these shelters, local authorities develop a plan for a future disaster scenario.

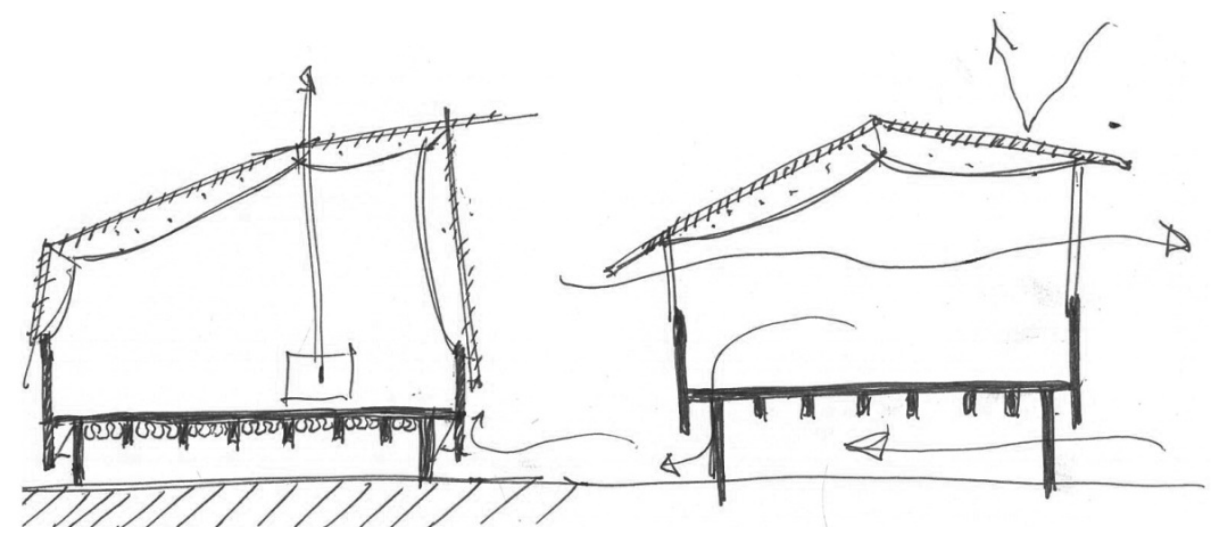

Figure 35 Sketches of deployment alterations based on climate

Once the emergency and relief stages draw to a close, a phase usually measured in weeks (Kates \& Pijawka, 1977; UN, 2004) further reconstruction can begin. 
This is again where planning is important, as the successful implementation of this stage requires two crucial aspects - the availability of materials and construction management. This next phase is focused on constructing, from the emergency shelter, a permanent core house. This will be small, just enough to provide sleeping and meal preparation spaces, but is the beginning of what will develop into a fully functioning home for the beneficiaries. How this is stage is managed is important and it is proposed that a supervision and educational system, similar to the one used in the Core Shelter Project, is implemented to ensure that homes can be built to appropriate standards; the opportunity to strengthen new buildings against future disasters is one that is often missed (Barakat, 2003).

Exactly how the components of the temporary shelter will form part of a permanent core is subject to local variation. The base has been designed to sit as a house base in itself, possibly only requiring that a sturdy structure and a waterproof facade and roof are applied to turn it into a permanent core; it can adapt beyond this however. The base can be raised on poles or stilts, should the house be located on uneven terrain, flood-prone ground, or simply require a small amount of clearance; it can also be supported and tied to solid footings, concrete, stone or brick. The role it plays is to provide a suitable floor for the core of the house; in the case that the base is not the best type of flooring then it can be used as an auxiliary space to the core - another room, a veranda or a semi open space. The shelter component is thought of in a similar manner. It can become an integral component of the permanent house but, especially because of the panel based nature of it, can just as easily form internal partitions and screens, or be used as a fence of balustrade.

\section{Outcome - The Deployment}

The final design concept begins long before the disaster event. For a single solution to be able to span over multiple stages there would have to be significant planning done pre-disaster - the manufacturing of the shelter deployment; how they are distributed to affected sites, and how they are 
distributed on site; who controls the distribution, and who is funding the construction of shelters. These are all issues that would determine the success or failure of this proposal, but they are also issues that are far too large and complex to be adequately addressed here. What is proposed is that they are manufactured locally, with local involvement to ensure their usefulness to the specific site and disaster. This would also allow alterations and changes to be made to the deployment based on what materials are prevalent and suitable, as well as being appropriate for the climate and terrain of the area.
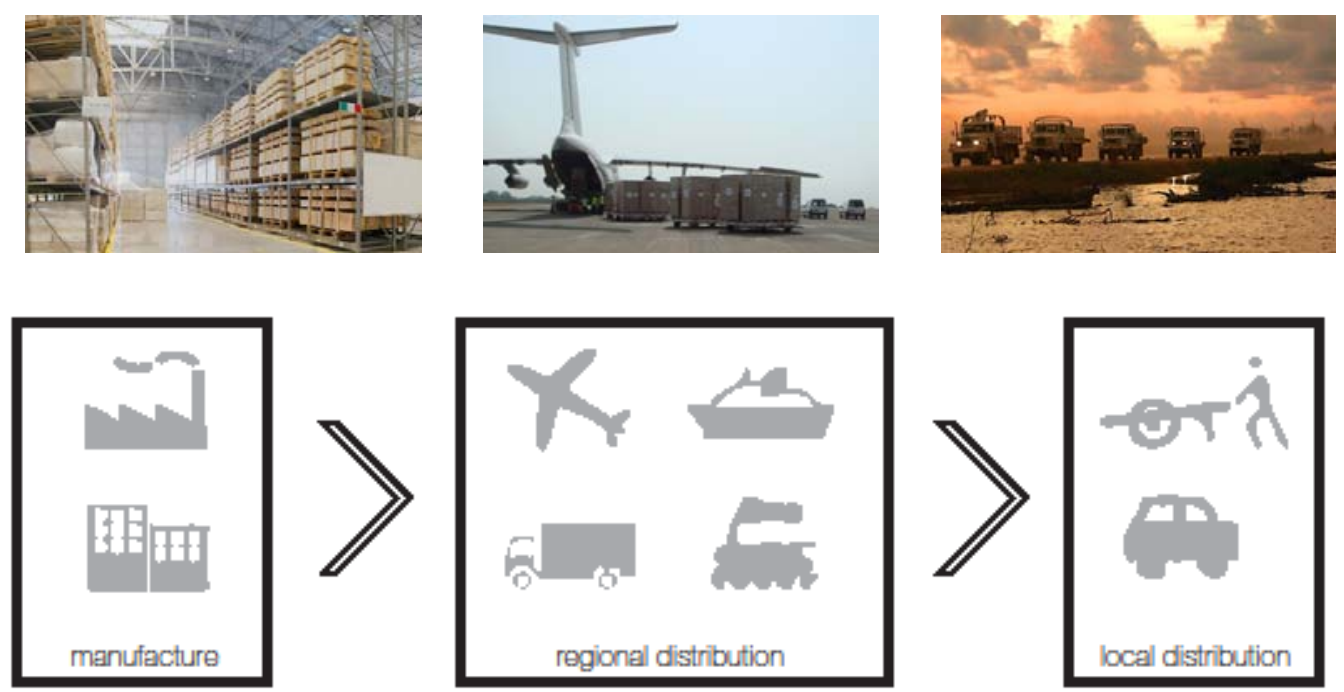

Figure 36 Disribution model. Manufacture at a national or regional level to enable quick deployment

Once the temporary shelters are distributed to site, they must be able to be transported to a suitable location and set up quickly and simply, with little or no expert assistance. The deployment consists of three main components - the base; the structure, or panels, and the cover.

The base is a simple joist platform. Alterations can be performed to make it more appropriate. Insulation can be added in between the structure to protect against the cold; it may be raised off the ground to provide ventilation in a hot climate, or to avoid flooding in a wet one. The base of the rails can be changed to support further adaption and use in the second stage - receiving posts, or being tied into a solid floor. 

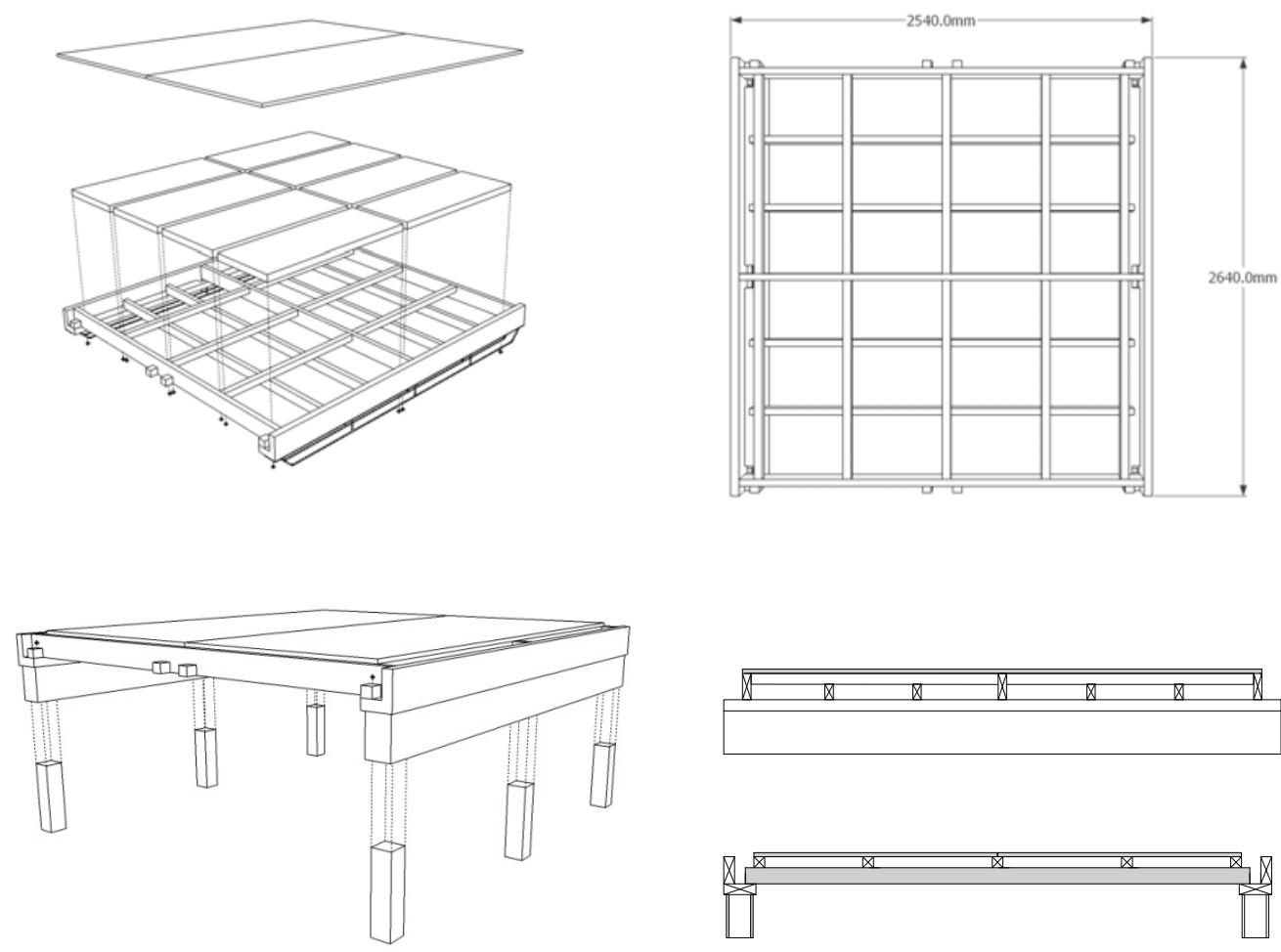

Figure 37 Construction of the base

The panels consist of two parts - the framing, which gives most of the strength to the panels, and the slats, which create formwork to add future materials onto or to create a semi-permeable screen for ventilation or privacy. Design of the panels allows them to be simply slotted into the base. Lateral support is gained from the base connection, interlocking the slats on the panels and from tying at corners. This gives a sturdy shelter that can be disassembled quickly and remade to suit as well as, crucially, being able to be added together to form larger structures. 

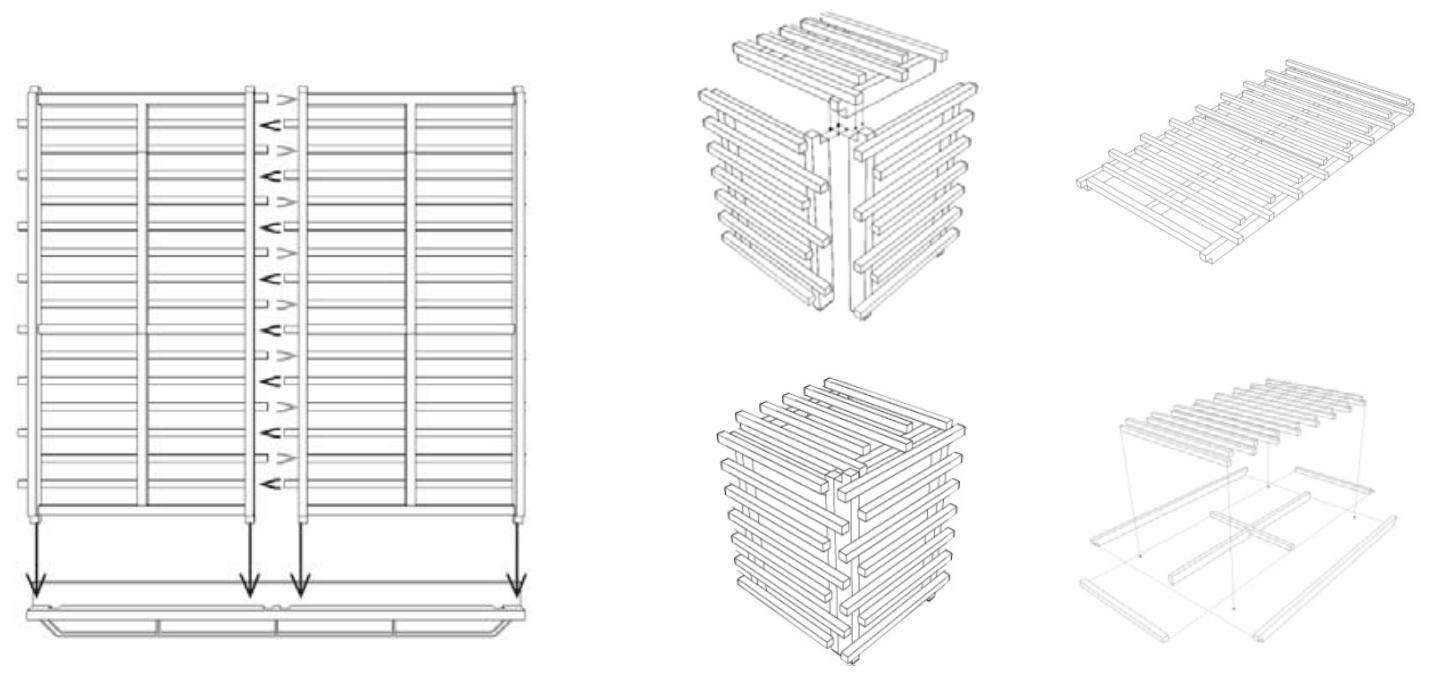

Figure 38 Panels slotting together, and into the base
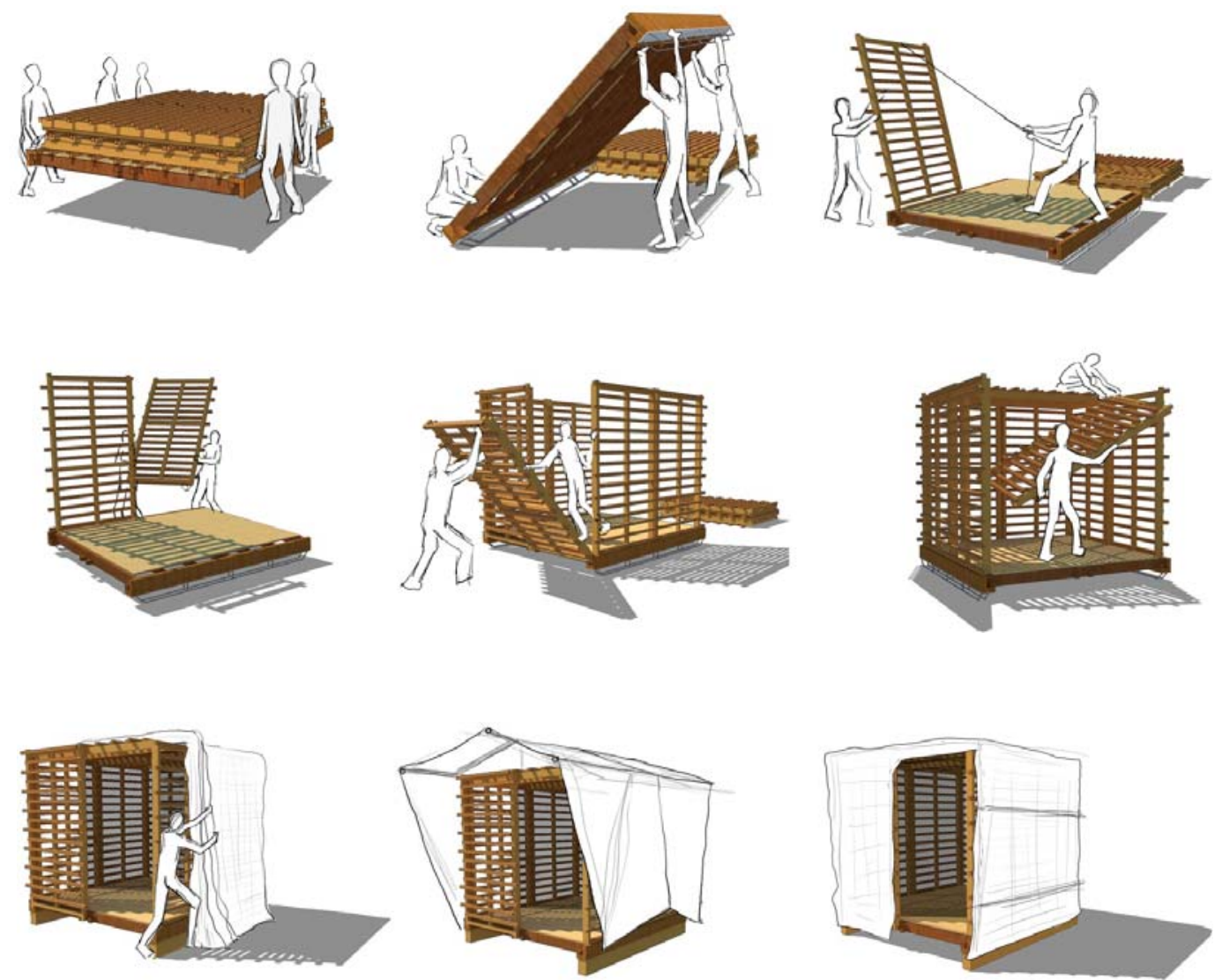

Figure 39 Step by step construction. Deployment put together by two or three people 
The final component of the shelter is the cover. Materiality will depend on what is available locally; it simply needs to provide shelter for the occupants by being waterproof. A fabric material, such as PVC vinyl fabric or canvas, would provide appropriate sheltering. The design would be reflective of the climate; pitched and open in tropical regions to provide ventilation and protection from heavy rain, double or triple skinned in temperate regions to insulate the inhabitants.

The shelter is approximately square in plan, the base measuring $2.7 \times 2.54 \mathrm{~m}$. The rectilinear plan was used for several reasons, the first two of which were touched on in the previous chapter. First, the rectangular plan is the prevalent type globally. Circular plans are common among nomadic peoples, or in larger community based societies, but not as prevalent in permanent communities. Secondly, this plan type can provide excellent lateral resistance when properly braced, giving the house resistance against future disasters. Finally, and advantageously over a circular plan, it can be easily tessellated. The base deployment, only $5.6 \mathrm{~m} 2$, is perhaps only suitable for two or three inhabitants, even on a temporary basis. The square plan allows multiple shelters to be joined together, in any configuration, to accommodate larger families or groups. With the provision of extra structure, relatively large spaces could be created very quickly. This means that the shelters may be used for other emergency functions; medical centres, search and rescue coordination, aid distribution; even makeshift community buildings such as schools or churches. 

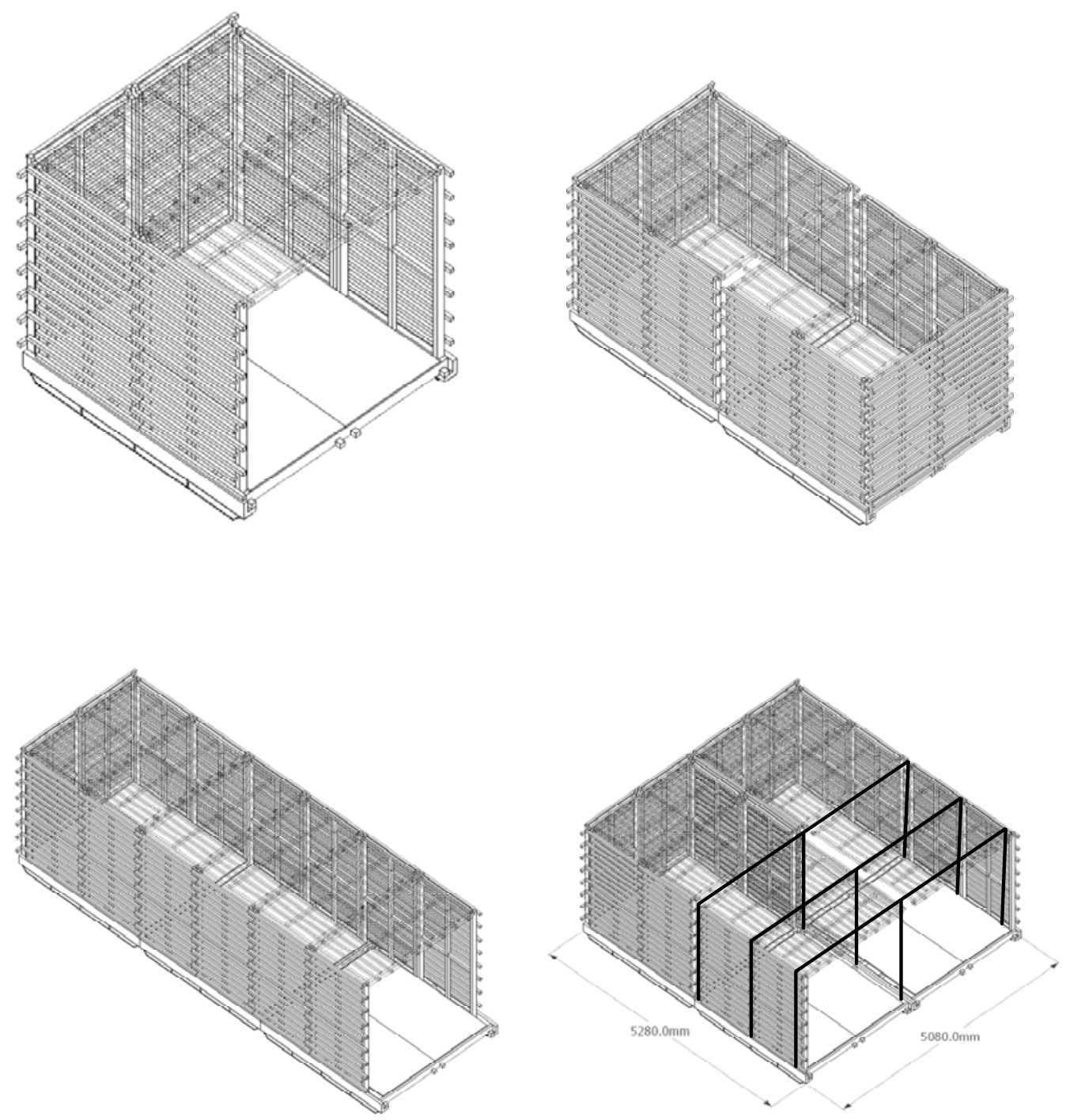

Figure 40 Tessellation of the basic deployment to create larger shelters

An important aspect of emergency shelters for disasters or refugees is their ability to the transported quickly and en masse. The solid panel and base system presented here takes up significantly more volume than does an IFRC or UN tent. It may simply be argued that this design is completely different; that it is a permanent housing solution and cannot be compared to temporary tents. It has usefulness beyond just temporary shelter and so has a greater intrinsic value. $A$ permanent solution such as this should not be reduced in volume to compete with existing shelters. However it still must be transported and so must fully justify its volume. The panels lay flat on top of each other, interlocking with the base to become a flat packed unit. In between every second set of panels there is 
a space of $135 \mathrm{~mm}$ high, a total volume of $1.8 \mathrm{~m} 3$ which would be used for delivery of other essential supplies - medical, first aid kits, blankets, cookers, tools or food; any number of items required during recovery.

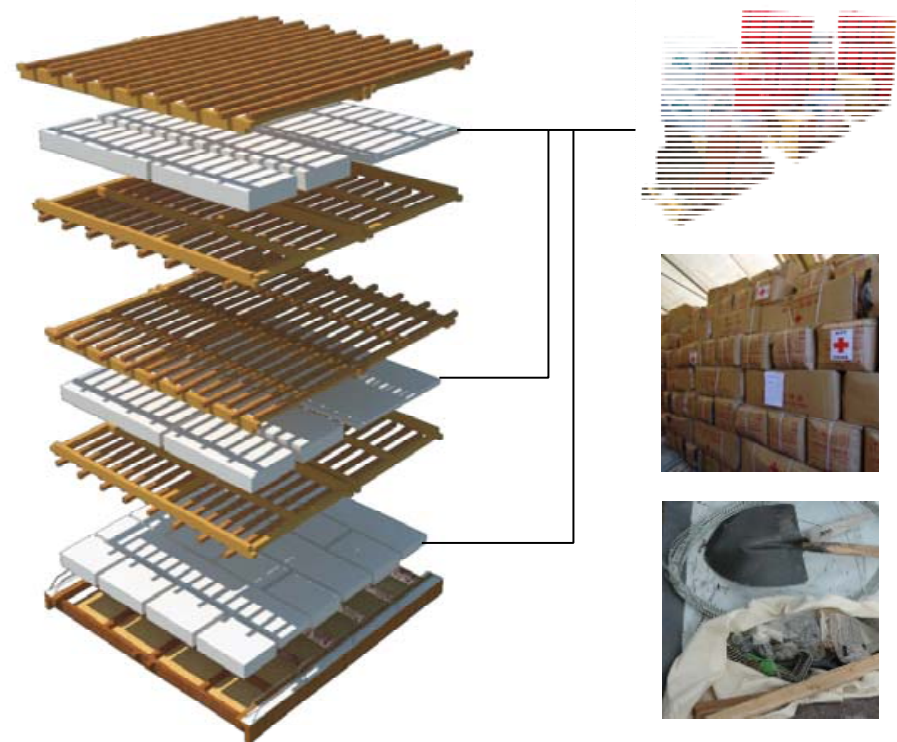

Figure 41 Packed down deployment. The white areas are space in between the panels

Finally, for the deployment stage, the shelter must be able to be taken apart again, or at least moved, to allow it to be located on its permanent site. This and allowing future changes is why the panels are not fixed more permanently to each other and the base, but instead tied and slotted.
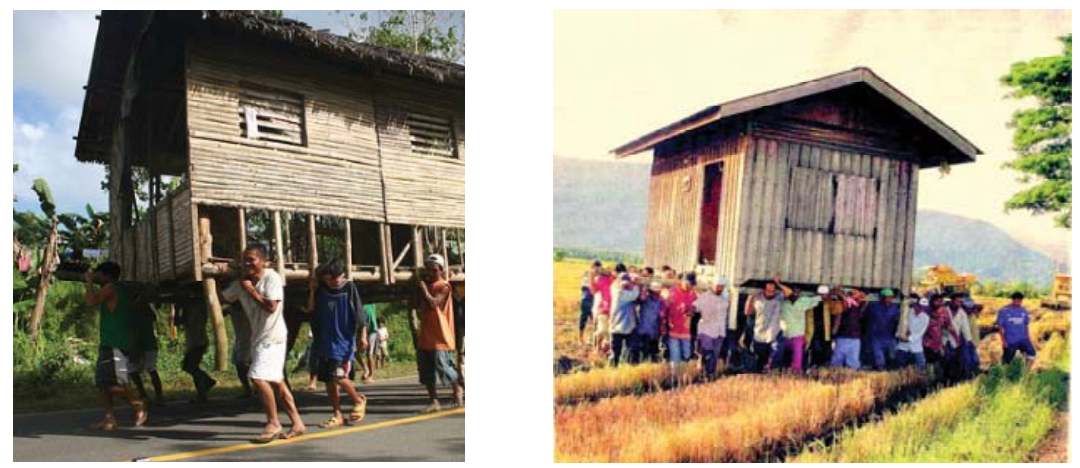

Figure 42 Small house or shelter transported to a new site 


\section{The Core Shelter}

Once the early phases of rescue and relief have been completed, processes of reconstruction can begin. The end of this phase will see major debris cleared from areas so the emergency deployments from phase 1 can be moved to permanent sites and construction can continue. Instead of waiting until all humanitarian operations have been completed and then waiting for development to begin on new housing, the beginning of the permanent dwelling is found in the temporary. The elements of the deployment - the base, panels and cover - support the construction of the core shelter, the first stage of the permanent house.

The idea is that by providing the base for a house in the emergency deployment, people can begin reconstructing what they have lost as soon as they are able to. Ideally, this would occur in tandem with local authorities and NGO's, having all been allowed for in pre-disaster planning; the organisations providing access to materials and technical knowledge. However very few things can be certain after a disaster, so this must also be accounted for. While it may be planned to construct houses from delivered materials, there may be delays, so the option of locally sourced or salvaged materials need also be considered. While the reconstruction may be overseen or assisted by authorities, the main driver of both the design and construction of the houses will be the community and the beneficiaries themselves. This is not only a source of available labour and knowledge, but gives focus to people who are struggling with loss and helps to install the finished home with a greater sense of ownership.

If we refer back to the houses of Düzce discussed in chapter four, Bektaş (2006) identifies four reasons the houses were altered by the inhabitants:

1. Function - adding new spaces, or partitioning existing spaces to allow for essential functions

2. Privacy - partitioning spaces to allow separation, particularly important for the predominantly Muslim population of Düzce

3. Environment - the addition of gardens for aesthetics, which also serves as a barrier for privacy and for providing food 
4. Activities - entrance and semi-open spaces for leisure

Additions are not neatly categorised into one reason or another - the garden space reflects almost all of the reasons. Rather they outline the main requirements a permanent house must fulfil in order to be suitable and useful. Stage 2 , the core house, may not be able to complete all these requirements for its inhabitants; here the deployment transforms, not into a finished product of a house, but the beginning of one.

Here the four sites outline in the previous chapter become useful. The conditions that persist in each provided parameters for what an emergency deployment would have to be and do to be useful as a permanent structure. The transition to the core house represents a largely functional move - applying a skin to the house, giving it foundations and enabling household routine to be resumed within.
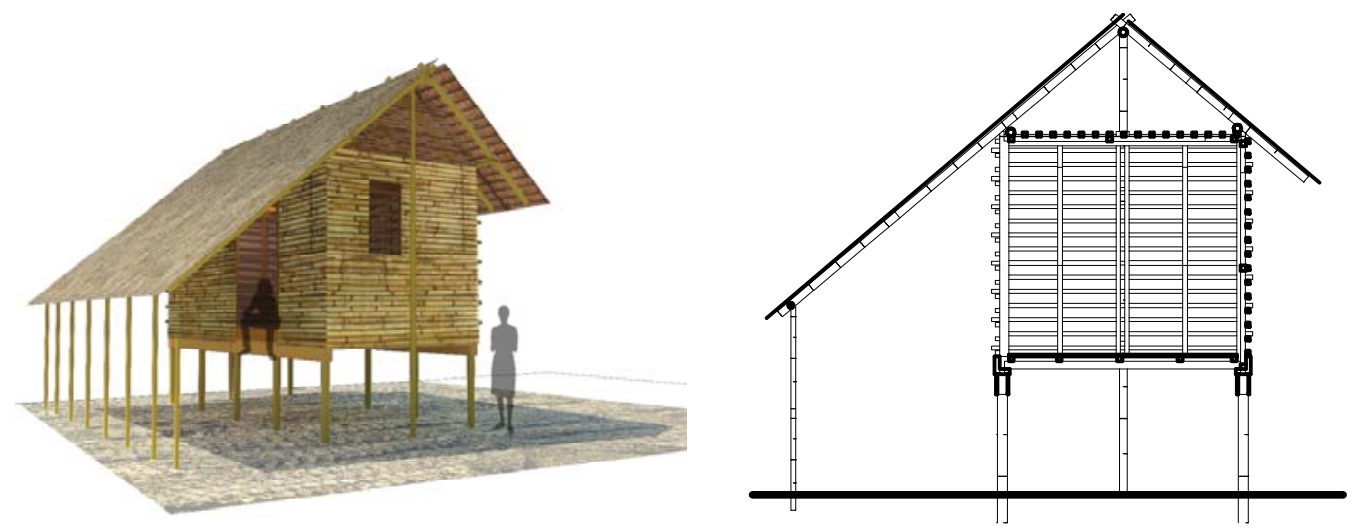

Figure 43 Modified core shelter, Iriga City

In Iriga City the form of the house is strongly defined by the climate. Monsoonal rains require significant eaves and covered outdoor spaces double as shelter from the tropical sun. Raised above ground level, the house is afforded ventilation beneath as well as being protected from the floods typhoons can bring. The interior of the house provides meal preparation and cooking space as well as becoming the sleeping area at night, equipment and possessions being stored above, in the loft space, or beneath the house. The presence of bamboo in tropical regions, coupled with its fast growth and inherent strength, makes it 
an ideal choice of structural material. Here more traditional claddings are imagined - palm thatching creating a watertight roof cladding and local timber for wall claddings - not because they may be the most preferred or ideal but because they are easily sourced and constructed in a situation where materials made further away may be unavailable for a time.

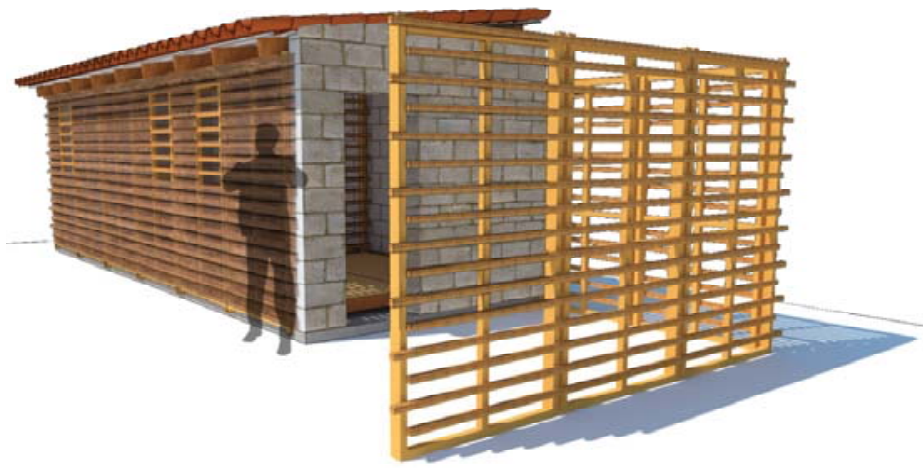

Ankara presents a much different climate; it experiences much less rain than Iriga and winter temperatures drop below zero, almost being categorised as cold desert

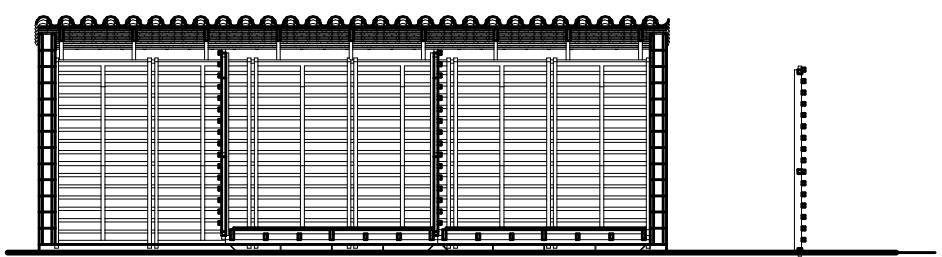

climate. As such, buildings are constructed from much more insulating materials; fired Figure 44 Modified core shelter, Ankara brick and adobe, reinforced block, or concrete. Here then, it is less appropriate for the panels to be permanent walls, perhaps instead forming partitioning of the interior space, or fencing outside for private outdoor areas. Floors tend to be solid so the base of the shelter, complete with insulation, may be tied into a poured concrete floor creating an interior floor protected from the cold of winter. The panels may also be used to form the roof. Pitches are less severe here because of the lower rainfall and, like many other places around the Mediterranean, the roof is clad in heavy clay tiles which may be lay and tied to the panels. 
Bhuj experiences even less rain than Ankara, receiving almost no rain for up to 8 months of the year; the other four a light rainy

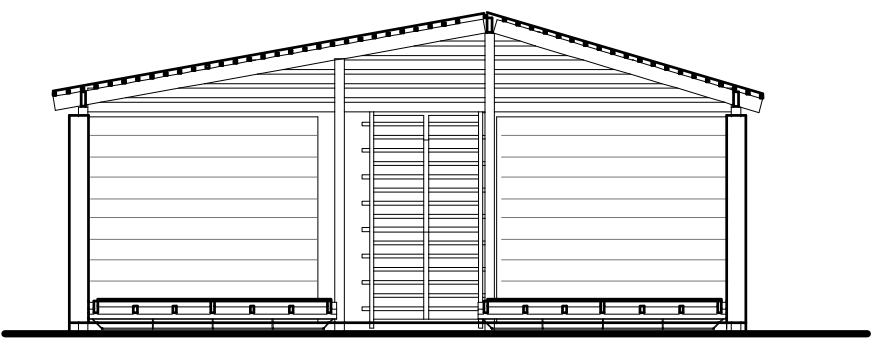
season. Being in a desert climate means timber is scarce and must be delivered from elsewhere in the sub-continent, so prevalent materials are ones made from the ground like brick, mud block or rammed earth, or concrete slab,

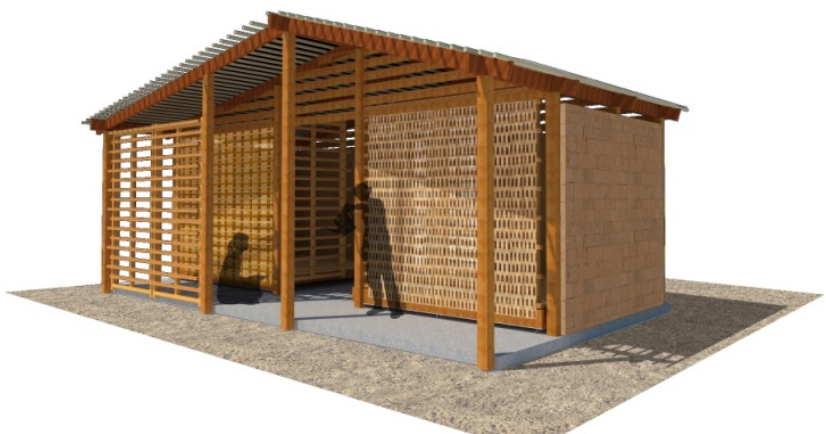

Figure 45 Modified core shelter, Bhuj block or corrugated iron. The house here uses panels to create screen open-air areas, used for meal preparation. They also allow ventilation that is necessary in such a hot, dry climate as well as allowing privacy through the application of woven mats to them; this has the added advantage of individualisation and decoration of the houses.

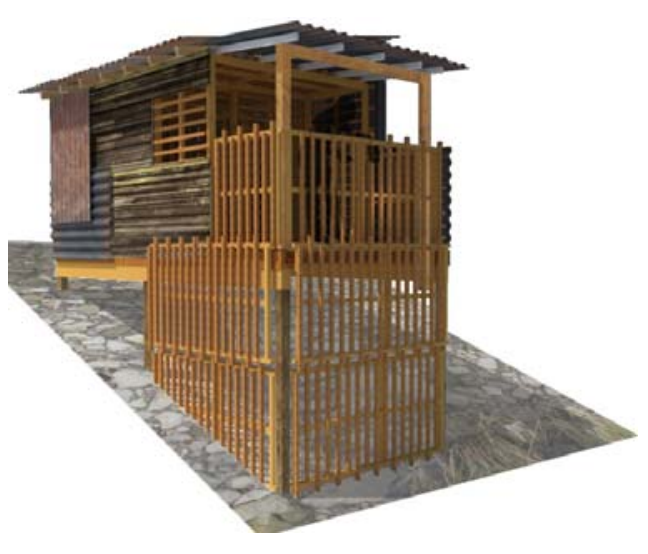

Rio de Janeiro's location puts it on the climatic border of tropical and temperate giving it a mild oceanic climate, reasonable warm all year round. More interestingly, Rio represents the informal settlements of many of the world's cities - set up hastily, sometimes overnight, without official approval or support. In Rio these are the

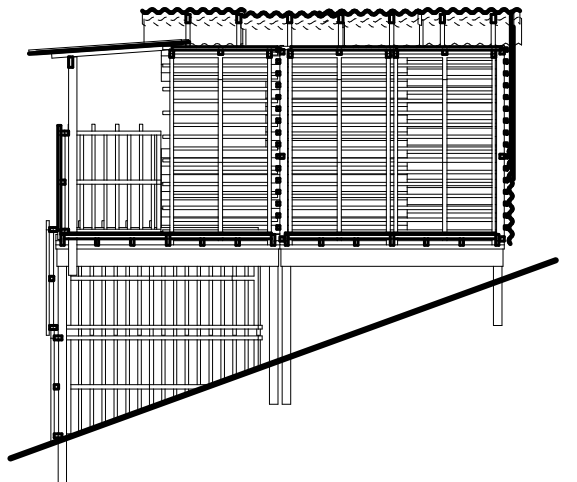

Figure 46 Modified core shelter, Rio de Janeiro
Favelas, perched on the steep hillsides surrounding the city and often the area's most affected by flooding and landslides during the wetter summers. Reconstruction taking place on such a site necessitates building onto poles, to give a flat floor but also to allow the flow of water down the hillside, reducing the risk of 
landslides. This site also addresses reconstruction from salvaged and found materials, which may be fixed as cladding to the supplied panel structure, or used to build new spaces off the core.

\section{Expansion}

While the core is the beginning of the permanent house, it is not the complete dwelling. The core shelter provides the basis for starting to resume a normal household routine - one of the less tangible losses from a major disaster. So the core may provide privacy, cooking and preparation space and sleeping areas for the inhabitants but it may not provide comparably to what was lost, "Housing is multi-faceted; a house is not simply a place of residence, but may also be a workplace and a means of conferring social status and standing in a community" (Barakat, 2003)

The core is built with the intention that it will be added onto. The recovery efforts will continue on for months or years replacing what was lost and as the community and society rebuild aspects, so can the household. Some things that were comparatively unimportant in earlier phases become paramount - repair and re-connection of utilities, repair of business and industry and repairing infrastructure. So the house will be developed as well. Storage spaces will be added, extra bedrooms, ablutions, and home-based business; the reconstruction driven by the requirements and resources of the inhabitants. The houses will be individualised and customised to so that they become what the inhabitants need them to be. 


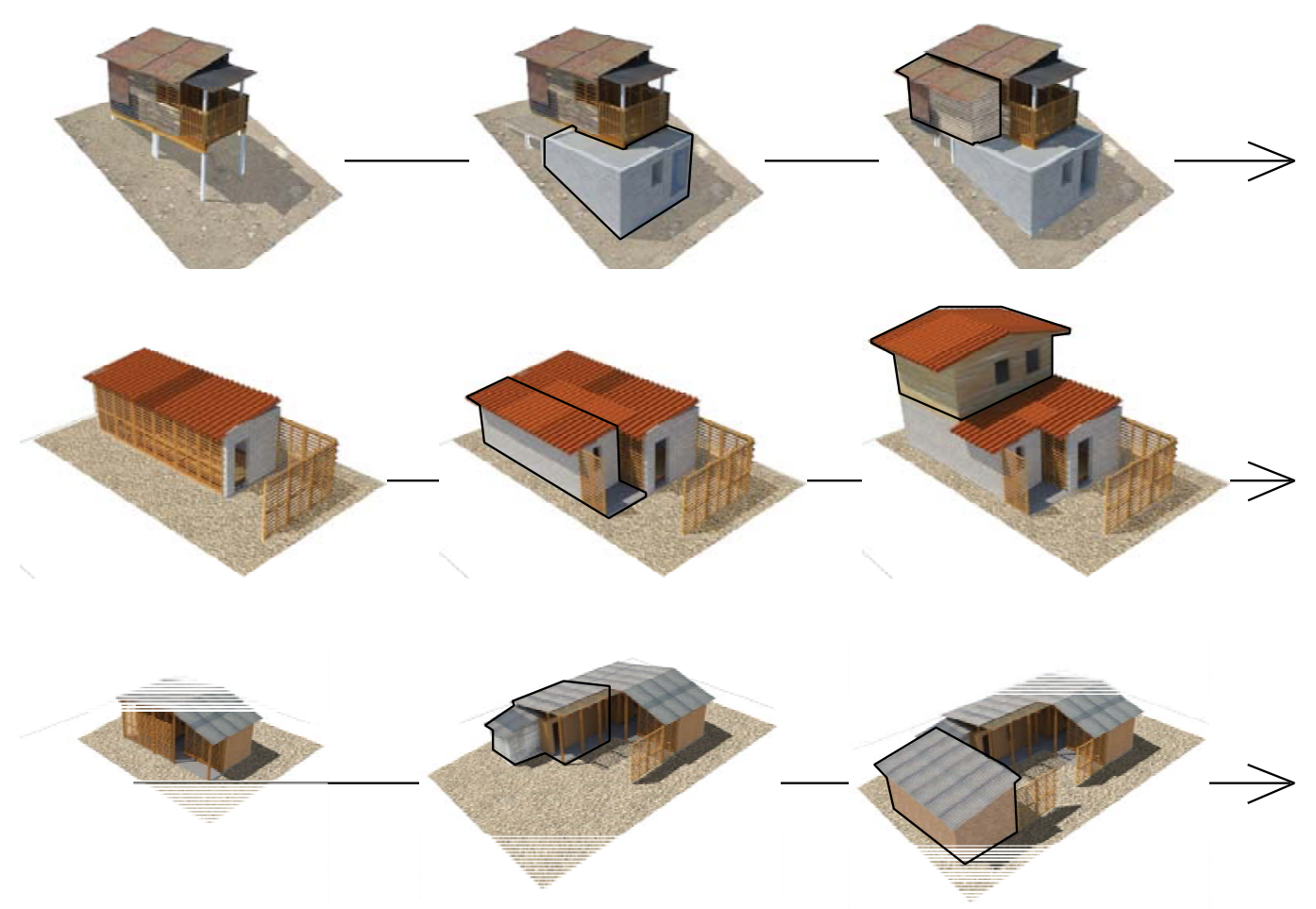

Figure 47 Speculative growth patterns.

The three stages proposed here may be thought of in terms of 'management' input, or how much input groups other than the affected have on each phase; each subsequent phase requiring less. The manufacture and deployment of the initial shelter relies on organisations and government to be successful; the core shelter requires instruction from a trained source to ensure the purpose of the shelter is conveyed and disaster resistant building techniques can be taught and employed, a transfer of knowledge. The expansion phase requires little management; indeed, as was seen in Düzce, it will often happen in spite of intentions, in order to fulfil requirements of the inhabitants.

Here it is appropriate to recall the needs discussed in Chapter Two. Identified were three distinct levels of human needs that were related to the built environment, and relevant to the reconstruction process - physical, cultural and social needs. All forms of shelter and housing address physical needs, adequately or not; indeed they must, as it is most critical to survival. Cultural needs may be addressed by permanent built solutions, giving security, better quality (than temporary shelters) or regular services; these aspects are necessary for a successful home, but unfortunately are not always present. And this statement 
can be considered even truer for the social needs of a house. As mentioned earlier, houses thought of as a product regularly fail to accommodate these higher level requirements; failure to account for daily routine and relationships in a household and community means that a house may not be appropriate, and this is why the third stage, the expansion, is so important. The idea of a hierarchy of needs is just that, a hierarchy. Until lower tier needs are met the ones above are less important, but they do become very important as the wider process of reconstruction goes on.

The third stage of the design proposed here is not a form, or prescribed space, but the notion, concept and plan for when the deployment is used, and core shelter is built, it is not the end of the reconstruction. The deployment is not a product, but a tool to be used in the process of reconstructing. 


\section{Chapter 7 - Summary and Conclusion}

\section{Answer to the Research Question}

The problem that was raised in chapter one, the issue that has driven this research and design, is of victims of disasters being housed in temporary shelter or housing for longer than was anticipated, for any variety of reasons. This raised the question of whether a deployable shelter could be developed that would be more than a temporary roof - could it become a base for a permanent house? The solution presented does not, and cannot, eliminate all of the issues that can cause delayed reconstruction; it is focused on meeting the basic universal needs of each stage and then being adaptable to specific climates, traditions and practices. The other question approached here is can a 'universal' solution be applied to a problem so influenced by local factors and if so, is it appropriate?

It was suggested in Chapter 2 and 3 that the problem of stagnation of re-housing was contributed to by differing focuses of the actors involved in reconstruction, creating a schism between humanitarian relief and developmental activities, where displaced peoples are moved from one shelter to another until the final house is delivered. The concept proposed here and discussed by others (Jigyasu, 2004; Baradan, 2006) is that housing reconstruction must be thought of as a process, not a product; this is what makes the adaptable, modular deployment appropriate, that each stage is not a different shelter or dwelling, but the same one built onto and modified.

Adaptive housing may be a solution to the problem, but how appropriate can a generic solution really be? The issues of applying a house to different locations, climates, cultures and disasters have been discussed in this thesis and applied to the proposed design; the deployment may be generic but adaptations will be made, especially to suit the climate to give people a basic level of comfort and shelter immediately after disaster and displacement.

So, if the deployment must be adapted to be more suitable, then why not completely tailor the deployment to the context and eliminate the generic 
element all together? This is certainly an option, potentially the most appropriate in some situations, but the strength of the generic deployment model is not its fixed form or style, but its role as a template. What it does do is simple, it can be easily deployed, quickly erected and meets basic shelter requirements; it is only when the basic form does not do this, that it would have to be altered. The stages that follow the initial deployment are focused on adaption and growth, to turn the generic shelter into a home that meets not only the physiological needs of the inhabitants, but the cultural and social ones too.

Finally, the success of this, or any reconstruction proposal, does rely heavily on planning. This has been discussed briefly, but not studied in this thesis; this limitation renders this not as a complete workable solution, but as an idea of how successful reconstruction may be approached. The reality is that once the disaster has occurred, it is much more difficult to introduce different plans or ideas; these need to be in place far in advance of any disaster taking place. "There is actually likely to be greater payoff by focusing on the pre rather than post impact period. Thus improvements are most likely if they are introduced into the pre-disaster development planning of a society" (Dynes \& Quarantelli, 2008).

\section{Limitations, Unanswered Questions and Implications}

Some of the limitations of this research were raised previously - namely the limited scope of the test sites being small and the speculative nature of much of the design. These parameters were imposed as necessary handicaps due to both the scale and timeframe of the project. This does mean however that because the design conclusions are speculative, very few certain conclusions can be drawn - that is, it cannot be stated definitively that this solution will be able to solve some, or any, of the issues it claims to; it still sits as an idea, a proposal.

This significant limitation creates an unanswered question of this concept: can the design solution actually do what is proposed? To be able to answer this, just 
as for any hypothesis, it must be the subject of experimentation. In this case it implies applying the design process to the problem - constructing and deploying the design into disaster scenarios. It is only once it can be studied in context that all the variables, largely human influenced ones, can be seen and judged.

Proposing that the design be tested in situations it has been specifically designed for may seem obvious but in order to be able to do this much more work would have to be done. How the physical design and construction operates and responds is a concern of the reconstruction process, but it is not the only one. It is probably not even a major one. The solution proposed in this research is dependent on many things: planning and collaboration between local and national government, between the government and professionals, the government and NGO's and, most crucially, between all these players and the affected people and communities. It is dependent on finding the support of all of these groups towards it. It is dependent on the economics of the solution related to other alternatives. It may depend on local policies and politics, especially in relation to disaster plans that may already be in place. There is a huge amount of management and planning work that would need to be undertaken, both generally and location specifically, in organising each group's role, because of paramount importance to the success of this reconstruction model is ensuring that those involved know what must be done and can do it when required. 


\section{$\underline{\text { References }}$}

Agarwal, A. (2007, March). Cyclone Resistant Building Architecture. UNDP, Disaster Risk Management Programme.

Allen, K. (2003). Vulnerability reduction and the community-based approach: A Philippines study. In Pelling, M. (Ed.) Natural disasters and development in a globalising world. Routledge : London.

Amin, S., \& Goldstein, M (2008) Data Against Disasters: Establishing Effective Systems for Relief, Recovery, and Reconstruction. Washington DC: The World Bank.

Architecture for Humanity (2010) Retrieved from http://architectureforhumanity.org/.

Arslan, H., Unul, A. (2006, May). The Evaluation of Community Participation in Housing Reconstruction Projects after the Düzce Earthquake. Presented at Post-Disaster Reconstruction: Meeting Stakeholder Interests, Florence, Italy. Retrieved from http://www.grif.umontreal.ca/pages/papersmenu2006.htm

Baradan, B. (2006, May) Analysis of the Post-Disaster Reconstruction Process following Turkish Earthquakes, 1999. Presented at Post-Disaster Reconstruction: Meeting Stakeholder Interests, Florence, Italy. Retrieved from http://www.grif.umontreal.ca/pages/papersmenu2006.htm

Barakat, S. (2003) Housing Reconstruction after Conflict and Disaster. Humanitarian Policy Network Paper 43. London: Overseas Development Institute.

Barenstein, J.D. (2006). Housing Reconstruction in Post-Earthquake Gujarat: A Comparative Analysis. Humanitarian Policy Network Paper 54. London: Overseas Development Institute.

Bektaş, E. (2006, May). A Post-Disaster Dilemma: Temporary Settlements in Dücze City, Turkey. Presented at Post-Disaster Reconstruction: Meeting Stakeholder Interests, Florence, Italy. Retrieved from http://www.grif.umontreal.ca/pages/papersmenu2006.htm

Çelebioğlu, B. \& Limoncu, S. (2006, May). Post-disaster Sustainable Housing Systems in Turkey. Presented at Post-Disaster Reconstruction: Meeting Stakeholder Interests, Florence, Italy. Retrieved from http://www.grif.umontreal.ca/pages/papersmenu2006.htm

Charleson, A.W. (2008). Seismic Design for Architects: Outwitting the Quake. Architectural Press: Oxford.

Charleson, A.W. \& Fyfe, G.D. (2001). Earthquake building damage in developing countries: a review of recent reconnaissance reports. Bulletin of the New Zealand National Society for Earthquake Engineering, 34(2). Pp 158-163.

Dartmouth Flood Observatory (DFO, 2010) 
Diacon, D. (1992, Sept). Typhoon Resistant Housing in the Philippines: The Core Shelter Project. Disasters 16(3). Pp 266-271. doi: 10.1111/j.1467-7717

Diacon, D. (1997). Typhoon Resistant Housing for the Poorest of the Poor in the Philippines. In Awotona, A. (Ed.) Reconstruction After Disaster: Issues and Practices. Ashgate: England.

Dilley, Maxx, Chen, R.S., Deichmann, U., Lerner-Lam, A.L., Arnold, M. (2005). Natural Disaster Hotspots: A Global Risk Analysis. Disaster Risk Management Series 5.

Washington, DC: World Bank.

Dynes, R.R., \& Quarantelli, E.L. (2008) A Brief Note on Disaster Restoration, Reconstruction and Recovery: A Comparative Note Using Post Earthquake Observations. Newark, Delaware: University of Delaware, Disaster Research Centre.

D'Urzo, S.J. (2002). Emergency and Architecture. Retrieved from ww.sandradurzo.org/Resources/Dossie_1.pdf

Enginoz, E.B. (2006, May) A Model for Post-Disaster Reconstruction: The Case Study In Dinar/Turkey. Presented t Post-Disaster Reconstruction: Meeting Stakeholder Interests, Florence, Italy. Retrieved from http://www.grif.umontreal.ca/pages/papersmenu2006.htm

Environmental Planning Collaborative [EPC] (2004, January) Participatory Planning Guide for Post-Disaster Reconstruction. Ahmedabad, India: Author.

Emergency Architects (2010) Retrieved from http://www.archi-urgent.com/

Federal Emergency Management Agency [FEMA] (1984) Objectives for local emergency management. Washington, DC: CPG 1-5.

Federal Emergency Management Agency (2005, September) Planning for Post-Disaster Recovery and Reconstruction. Washington, DC: Author.

Fritz, C.E. (1961) Disaster and community therapy. Washington, DC: National Research Council, National Academy of Sciences.

Halvorson, S.J., \& Hamilton, J.P. (2009) In the aftermath of the Qa'yamat: the Kashmir earthquake disaster in northern Pakistan. Missoula, Montana: The University of Montana, Department of Geography.

Heide, E.A. (1989) Disaster Response: Principles of Preparation and Coordination. St. Louis: CV Mosby.

Hicks, K.E., \& Pappas, G. (2006) Coordinating Disaster Relief after the South Asian Earthquake. Society 43(5). 42-50. doi:10.1007/BF02687574

Hogg, S.J. (1980) Reconstruction Following Seismic Disasters in Venzone, Friuli. Disasters 4(2). Pp 173-185. doi:10.1111/j.1467-7717.1980.tb00271.x

Huber, R., Langsaeter, T., Eggenhofer, P., Freire, F., Grilo, A. (2008) Part Two: The Case Study of Aceh and North Sumatra. NATO Research and Technology Organisation, Systems Analysis Studies. 
Internal Displacement Monitoring Center (IDMC). (2006). Disaster Induced

Displacement. Retrieved from http://www.internal-

displacement.org/8025708F004BE3B1/(httpInfoFiles)/C753862FA2CF8B7CC1257115004

752ED/\$file/Protection\%20from\%20module\%20handout\%20development\%20displace

$\underline{\text { ment.pdf }}$

International Federation of Red Cross and Red Crescent Societies (2007, March)

Operations Update, Pakistan: Earthquake. Appeal no.05EA022. Operations Update no.

28. Pakistan.

International Federation of Red Cross and Red Crescent Societies (2010) Disaster Management: Responding to Disasters. Retrieved from

http://www.ifrc.org/what/disasters/responding/index.asp

Jain, S.K., Murty, C.V.R., Chandak, N. (1994) The September 29, 1993, M6.4 Killari, Maharashtra Earthquake in Central India. EERI Special Earthquake Report, EERI Newsletter 28(1).

Jha, A.K., Barenstein, J.D., Phelps, P.M., Pittet, D., Sena, S. (2010). Safer Homes, Stronger Communities: A Handbook for Reconstructing after Natural Disasters. Washington DC: The International Bank for Reconstruction and Development / The World Bank.

Jigyasu, R. (2004, April) Sustainable Post-Disaster Reconstruction through Integrated Risk Management - The Case of Rural Communities in South Asia. Paper presented at 2004 International Conference and Student Competition on post-disaster reconstruction "Planning for reconstruction", UK. Retrieved from http://www.grif.umontreal.ca/pages/papersmenu2004.htm

Kakumbi, G.M. (2004, April) Traditional versus Modern Reconstruction Practices. Paper presented at 2004 International Conference and Student Competition on post-disaster reconstruction "Planning for reconstruction", UK. Retrieved from http://www.grif.umontreal.ca/pages/papersmenu2004.htm

Kates, R.W., \& Pijawka, D. (2001) From rubble to monument: The pace of reconstruction. In Haas, J.E., Kates, R.W., \& Bowden, M. (Eds.) Reconstruction following disaster. Cambridge, MA: MIT Press.

Kendra, J.M., \& Wachtendorf, T. (2007) Community Innovation and Disasters. In Rodriguez, H., Quarantelli, E.L., Dynes, R. (Eds) Handbook of Disaster Research. New York: Springer.

Kilburn, C., \& McGuire, B. (2001) Italian Volcanoes. UK: Terra Publishing.

Khan, H.U., \& Moore, C. (1990) The Architecture of the Individual House. In R. Powell (Ed.) The Architecture of Housing (pp. 166-183). Singapore: Concept Media/The Aga Khan Award for Architecture.

Lonely Planet (2010). Images. Retrieved from http://www.lonelyplanetimages.com/images/ 
Mahmood, A. (2009) Earthquake Vulnerability Assessment: Pakistan 2005-06.

Islamabad: The Population Council. Retrieved from

http://www.popcouncil.org/projects/RH_PakEarthquake.html

Maslow, A.H. (1943) A Theory on Human Motivation. Psychological Review 50(4). Pp 370. doi:10.1037/h0054346.

Make It Right (2007) Helping to Rebuild New Orleans Ninth Ward. Retrieved from http://www.makeitrightnola.org/

Malik, R. (July 2001). To Rebuild Kutch. Hinduism Today July/August 2001. Retrieved from http://www.hinduismtoday.com/modules/smartsection/item. php?itemid=4063.

Murty, C.V.R., Jain, S.K., Sheth, A.R., Jaiswal, A., Dash, S.R. (2006) Response and Recovery in India after the December 2004 Great Sumatra Earthquake and Indian Ocean Tsunami. Earthquake Spectra 22(3). Pp 731-758. doi:10.1193/1.2206137

National Commission on Indigenous Peoples [NCIP] (2010). Core Shelter Proposal Pictures. Retrieved from http://iriga.net/projects.html

Nikhileswarananda, S. (2004, April) Post-Disaster Reconstruction Work in Gujarat. Paper presented at 2004 International Conference and Student Competition on post-disaster reconstruction "Planning for reconstruction", UK. Retrieved from http://www.grif.umontreal.ca/pages/papersmenu2004.htm

The National Commission for Indigenous People [NCIP]. (2006, Dec). Core Shelter Project Questions and Answers. Retrieved from www.iriga.net .

Oliver, P. (1987). Dwellings: The House across the World. Oxford: Phaidon Press Limited; London.

Oliver, P. (2007). Atlas of Vernacular Architecture of the World. Routledge; London.

Oliver, P. (1997). Encyclopedia of Vernacular Architecture of the World. Cambridge University Press; Cambridge.

Peacock, W.G., Dash, N., Zhang, Y. (2007) Sheltering and Housing Recovery Following Disaster. In Rodriguez, H., Quarantelli, E.L., Dynes, R. (Eds) Handbook of Disaster Research. New York: Springer.

Perry, R.W. (2007) What is a Disaster? In Rodriguez, H., Quarantelli, E.L., Dynes, R. (Eds) Handbook of Disaster Research. New York: Springer.

Phister, Dr P.W., Allen, Dr D., Barath, Dr J., Brandenburger, U. (2009) Pakistan Earthquake Case Study. Washington, DC: Department of Defence Command and Control Research Program.

Potangaroa, R. (2006, May) Gimme Shelter: Tsunami Mitigation as Part of a Permanent Shelter Program for Aceh, North Sumatra. Presented at Post-Disaster Reconstruction: Meeting Stakeholder Interests, Florence, Italy. Retrieved from http://www.grif.umontreal.ca/pages/papersmenu2006.htm 
PRNC (1994). PRNC: strategies towards the $21^{\text {st }}$ century: improving the situation of the most vulernable. Unpublished: Manila. As cited in Allen, 2003.

Quarantelli, E.L. (1989) Conceptualizing disasters from a sociological perspective. International Journal of Mass Emergencies \& Disasters, 7(3).

Quarantelli, E.L. (1995) Patterns of Sheltering and Housing in US Disasters. Disaster Preventions and Management 4(3). Pp 43. doi:10.1108/09653569510088069

Rapoport, A. (1984). Culture and the Urban Order. In Agnew, John, et. al. 1984, The city in Cultural Context, Allen \& Unwin Inc. Winchester, Mass.

Sassu, M. (2007). Vernacular Housing Construction. University of Piza; Italy.

Sinclair, C., \& Stohr, K. (Eds.). (2006). Design Like You Give a Damn. New York: Metropolis Books.

Tierney, K.J. (1985, January) Emergency medical preparedness and response in disasters: the need for interorganizational coordination. In Petak, W.J. Emergency management: a challenge for public administration. Public Admin Rev 45:77.

Texas Division of Emergency Management [TDEM] (2001) Disaster Recovery Manual. Austin: Texas Department of Public Safety.

Tierney, K.J., Lindell, M.K., \& Perry, R.W. (2001) Facing the unexpected: Disaster preparedness and response in the United States. Washington, DC: Joseph Henry Press.

Travel Blog (2010). Traditional Iban Longhouse. Retrieved from http://www.travelblog.org/Photos/2088769

United Nations Educational Scientific and Cultural Organisation [UNESCO] (2010). News Service. Retrieved from http://portal.unesco.org/en/ev.phpURL ID=42898\&URL DO=DO TOPIC\&URL SECTION=201.html

United Nations High Commission for Refugees [UNHRC] (2005) Handbook for Self Reliance. Geneva: Reintegration and Local Settlement Section, Division of Operational Support.

United Nations High Commission for Refugees [UNHRC] (2010). Photo Galleries. Retrieved from http://www.unhcr.org/pages/49c3646c25d.html

United Nations (2004). International Strategy for Disaster Reduction [UNISDR]. Geneva: Author.

United States Air Force [USAF] (2010). Photo Archive. Retrieved from http://www.af.mil/photos/index.asp

Vellinga, M., Oliver, P., Bridge, A. (2007). Atlas of Vernacular Architecture of the World. Routledge: New York.

Vos, F., Rodriguez, J., Below, R., Guha-Sapir, D. (2010). Annual Disaster Statistical Review 2009. Brussels: Centre for Research on the Epidemiology of Disasters (CRED). 
Wahlroos, V. (1999) Let People Build! Sustainable Approaches to Shelter. Habitat Debate 5(2). UNHCR.

What If New York City... (2008) Post Disaster Design Housing Competition. Retrieved from http://www.nyc.gov/html/whatifnyc/html/home/home.shtml

Wilford, J. (2008). Out of rubble: natural disaster and the materiality of the house. Environment and Planning D: Society and Space v26. Pp 647-662. doi:10.1068/d4207

Yunus, M. (2007). Credit for the poor: Poverty as distant history. Harvard International Review 29(3). 


\section{Appendix}
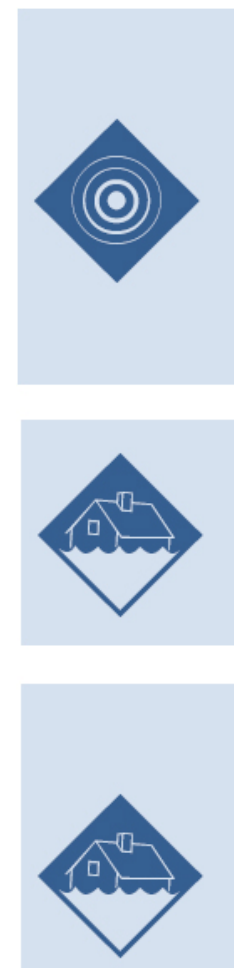

Earthquake: Shaking and displacement of ground due to seismic waves. This is the earthquake itself without secondary effects. An earthquake is the result of a sudden release of stored energy in the Earth's crust that creates seismic waves. They can be of tectonic or volcanic origin. At the Earth's surface they are felt as a shaking or displacement of the ground. The energy released in the hypocenter can be measured in different frequency ranges. Therefore there are different scales for measuring the magnitude of a quake according to a certain frequency range. These are: a) surface wave magnitude (Ms); b) body wave magnitude (Mb); c) local magnitude (ML); d) moment magnitude (Mw).

Flash flood: Rapid inland floods due to intense rainfall. A flash flood describes sudden flooding with short duration. In sloped terrains the water flows rapidly with a high destruction potential. In flat terrains the rainwater cannot infiltrate into the ground or run off (due to small slope) as quickly as it falls. Flash floods typically are associated with thunderstorms. A flash flood can occur at virtually any place.

General flood: Gradually rising inland floods (rivers, lakes, groundwater) due to high total depth of rainfall or snowmelt. A general flood is caused when a body of water (river, lake) overflows its normal confines due to rising water levels. The term general flood additionally comprises the accumulation of water on the surface due to long-lasting rainfall (water logging) and the rise of the groundwater table above surface. Furthermore, inundation by melting snow and ice, backwater effects, and special causes such as the outburst of a glacial lake or the breaching of a dam are subsumed under the term general flood. General floods can be expected at certain locations (e.g. along rivers) with a significantly higher probability than at others.

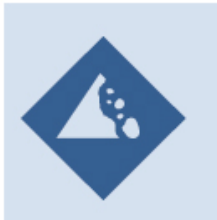

Landslide: Any kind of moderate to rapid soil movement including lahar, mudslide and debris flow. A landslide is the movement of soil or rock controlled by gravity and the speed of the movement usually ranges between slow and rapid. It can be superficial or deep, but the materials have to make up a mass that is a portion of the slope or the slope itself. The movement has to be downward and outward with a free face.

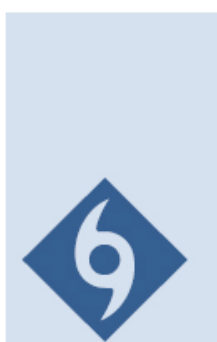

Tropical cyclone: A tropical cyclone is a non-frontal storm system that is characterized by a low pressure centre, spiral rain bands and strong winds. Usually it originates over tropical or sub-tropical waters and rotates clockwise in the southern hemisphere and counter-clockwise in the northern hemisphere. The system is fuelled by heat released when moist air rises and the water vapour it contains condenses ("warm core" storm system). Therefore the water temperature must be $>27{ }^{\circ} \mathrm{C}$. Depending on their location and strength, tropical cyclones are referred to as hurricane (western Atlantic/eastern Pacific), typhoon (western Pacific), cyclone (southern Pacific/Indian Ocean), tropical storm, and tropical depression (defined by wind speed; see Saffir-Simpson-Scale). Cyclones in tropical areas are called hurricanes, typhoons and tropical depressions (names depending on location).

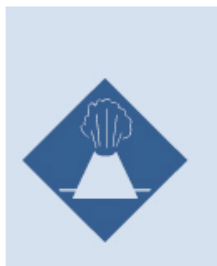

Volcanic eruption: All volcanic activity like rock fall, ash fall, lava streams, gases etc. Volcanic activity describes both the transport of magma and/or gases to the Earth's surface, which can be accompanied by tremors and eruptions, and the interaction of magma and water (e.g. groundwater, crater lakes) underneath the Earth's surface, which can result in phreatic eruptions. Depending on the composition of the magma, eruptions can be explosive and effusive and result in variations of rock fall, ash fall, lava streams, pyroclastic flows, emission of gases etc.

Figure 48 Definitions of disasters. (Vos, et al, 2010) 


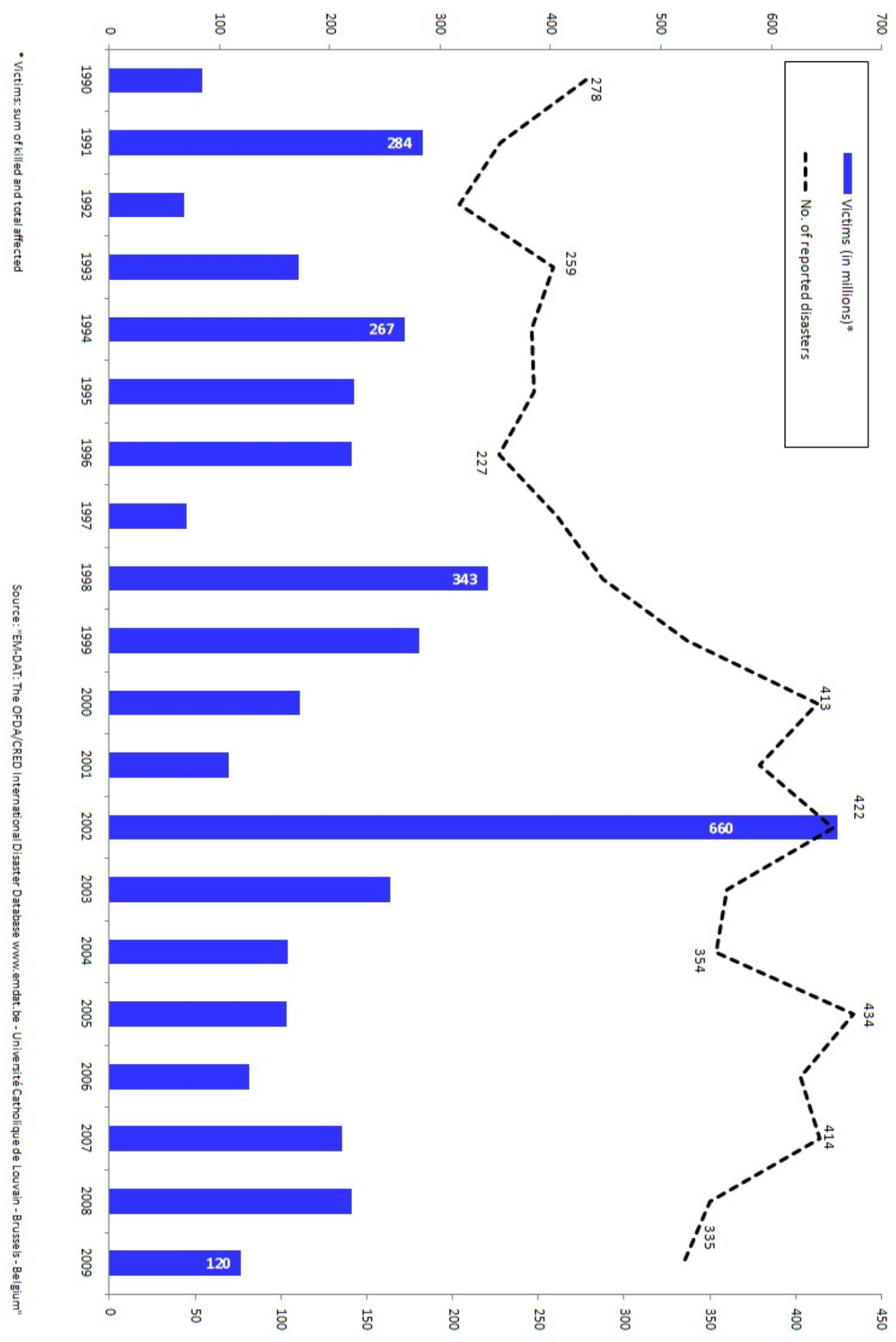

Figure 49 Trends in occurrence and victims (Vos et al, 2010) 


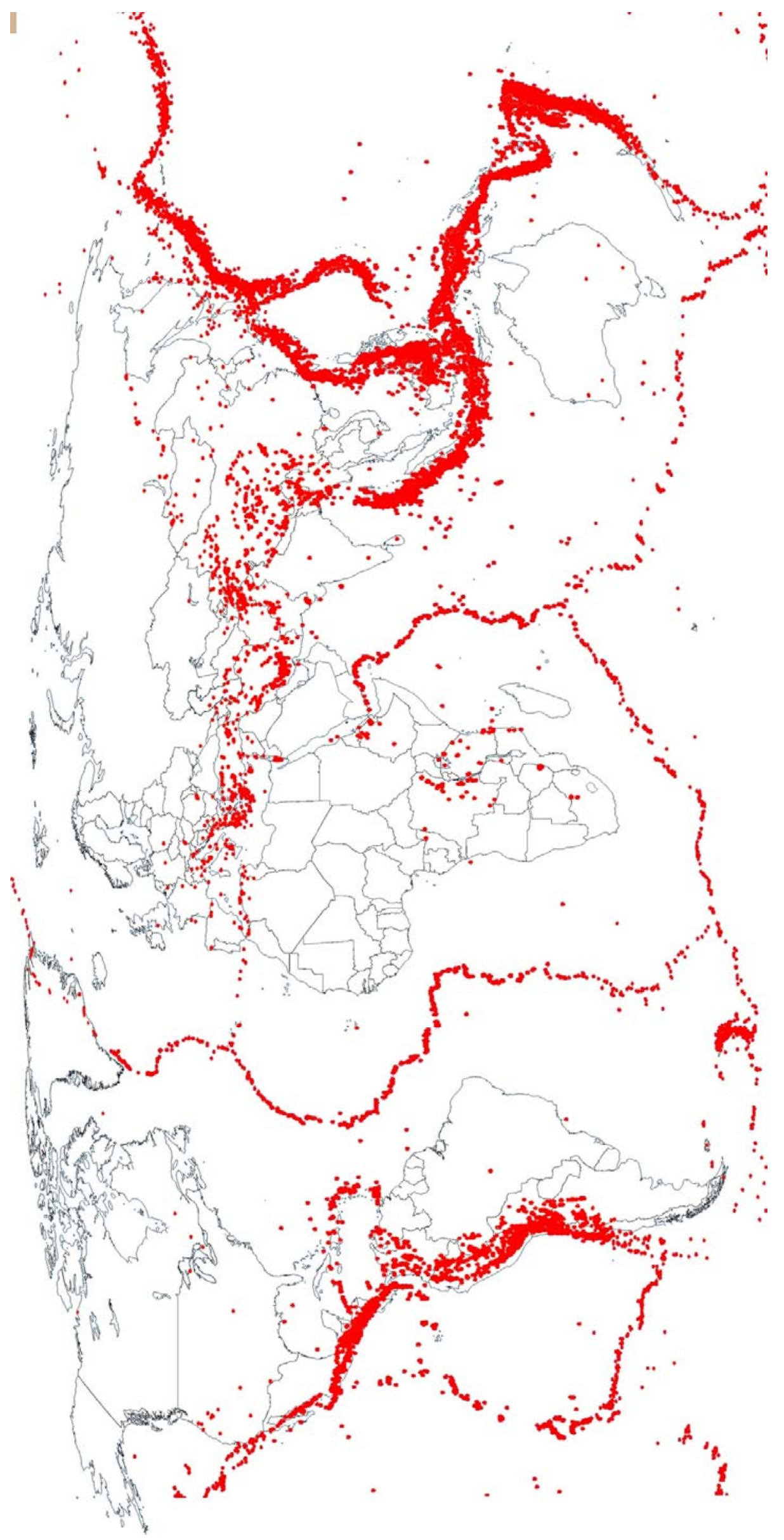

Figure 50 Earthquakes 


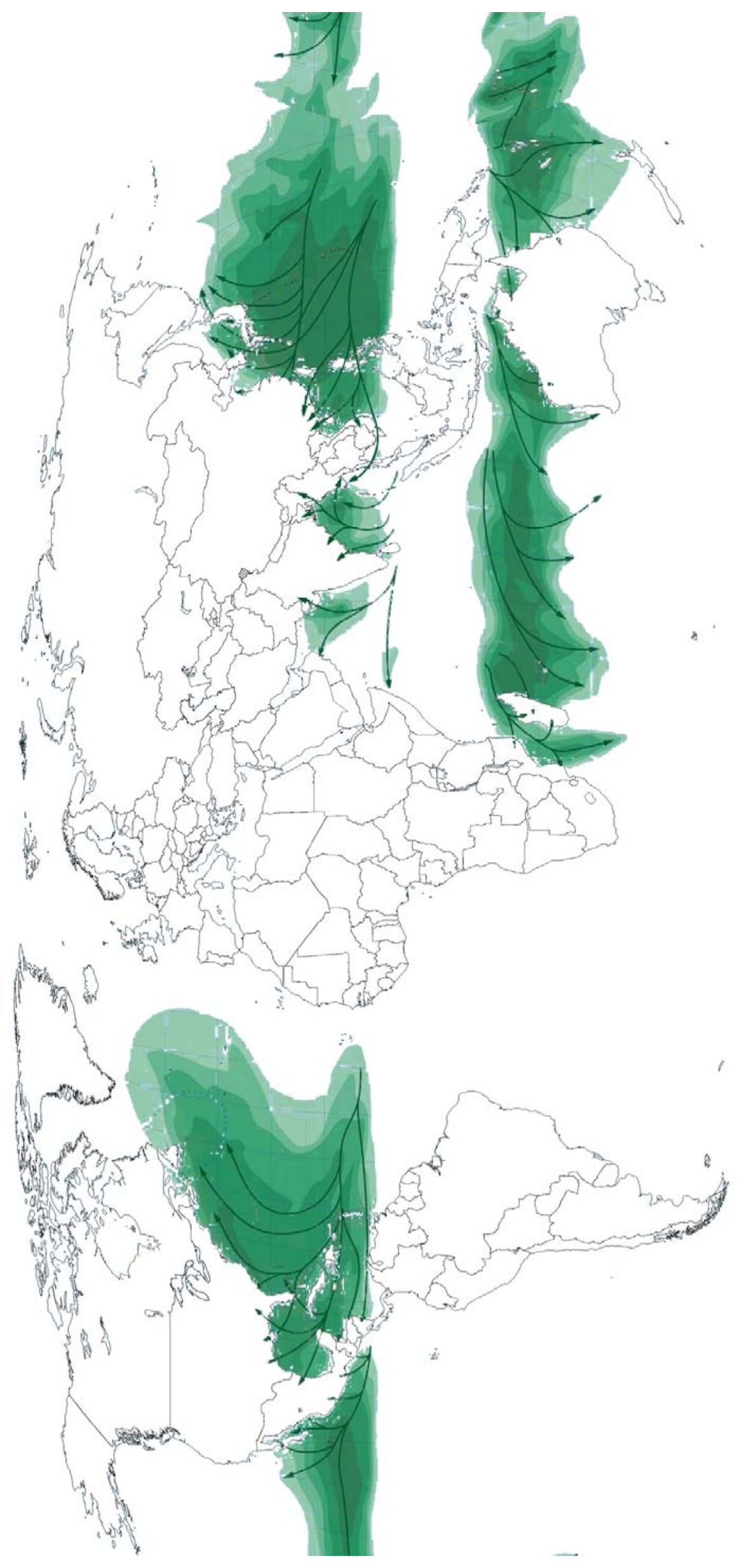

Figure 51 Cyclones 


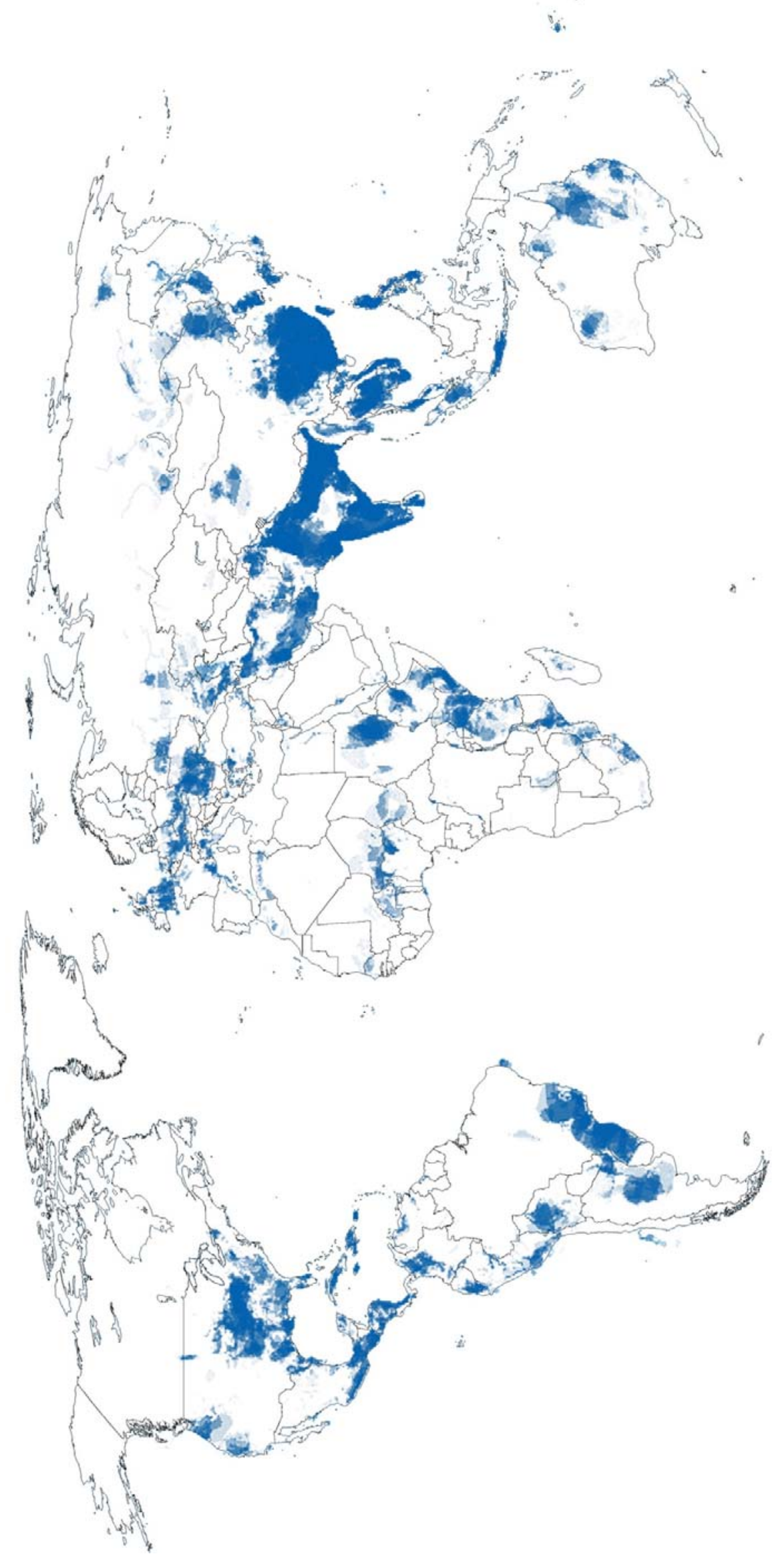

Figure 52 Flooding 


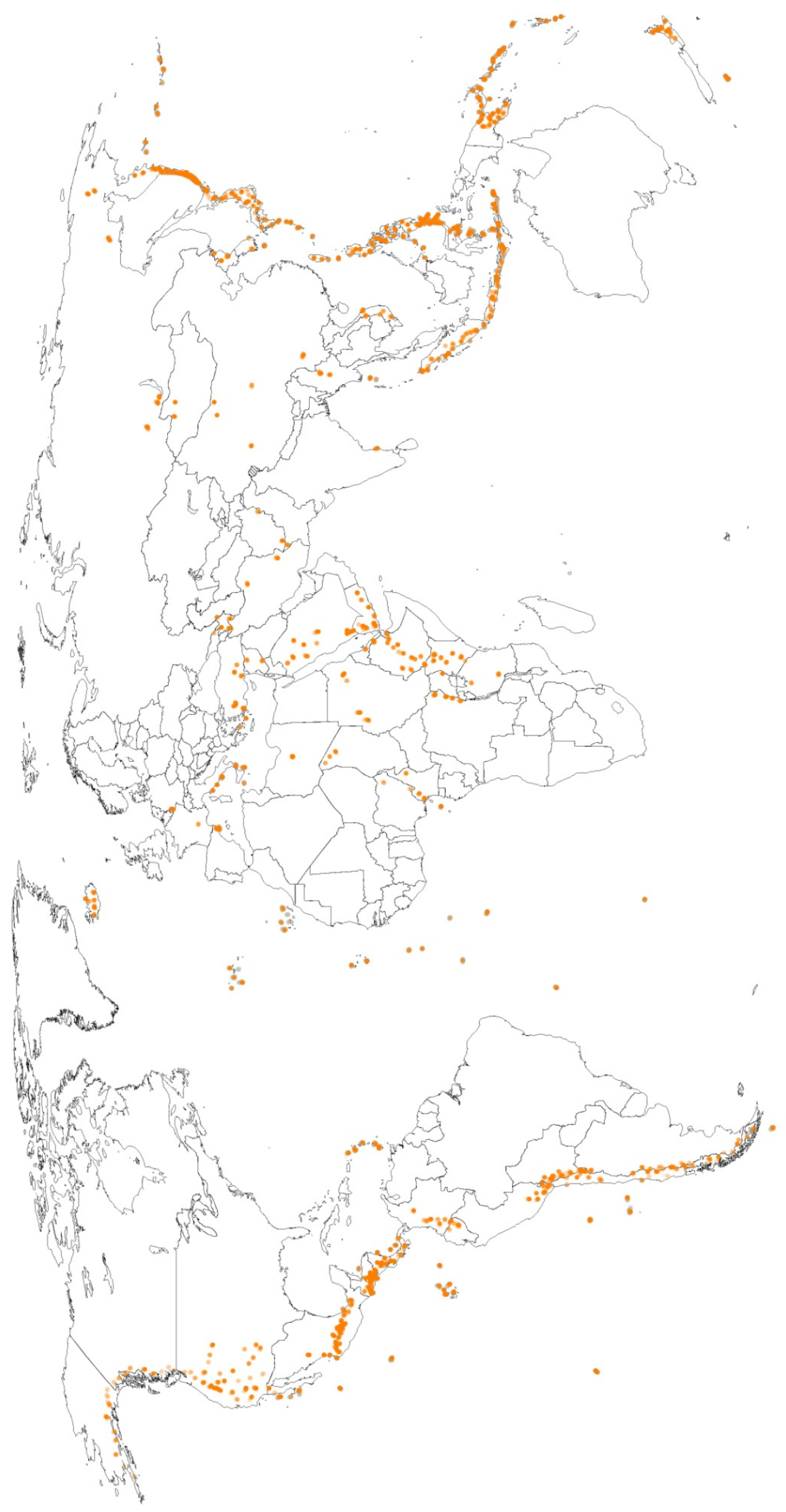

Figure 53 Volcanoes 


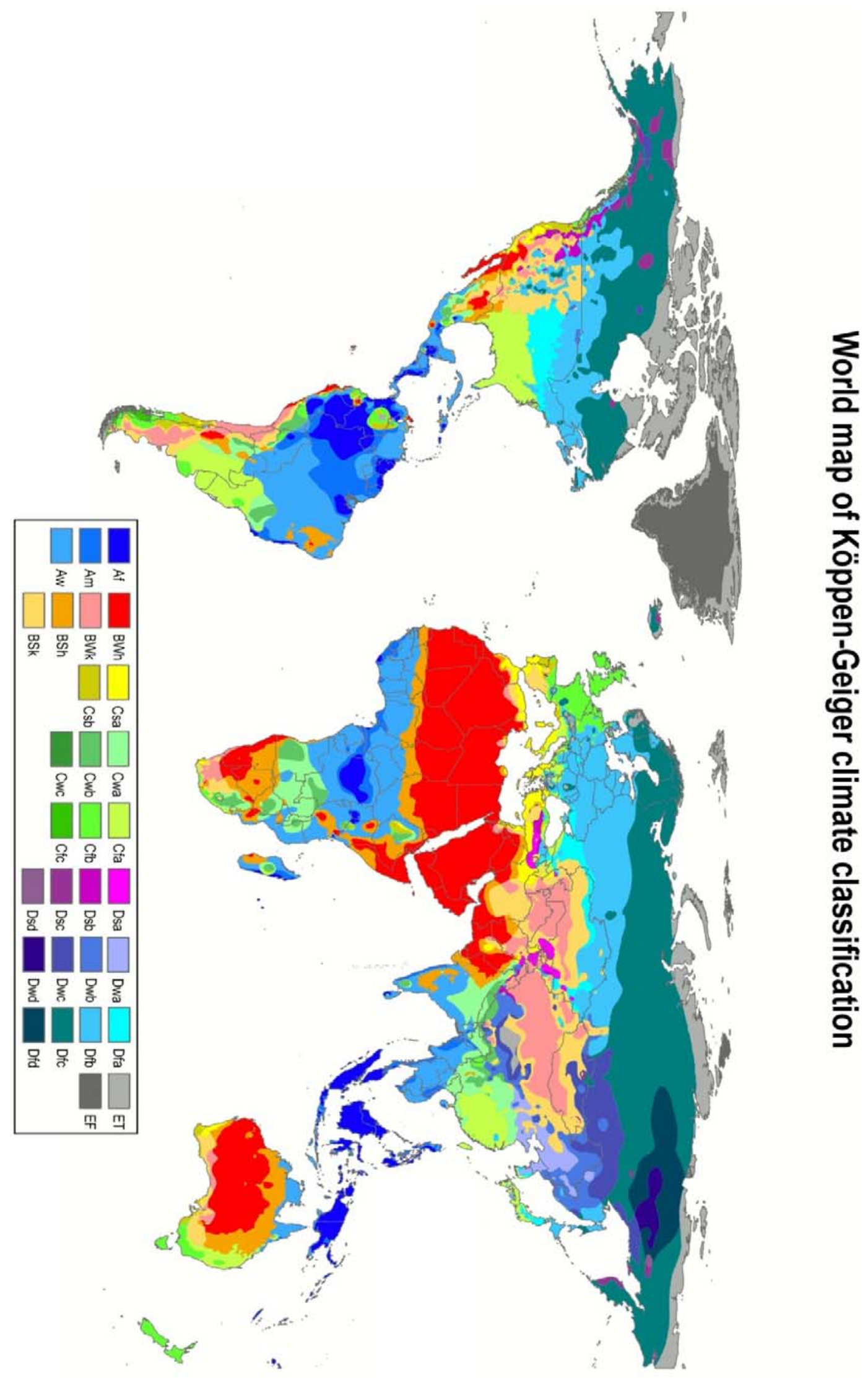

Figure 54 Köppen-Geiger Climate Classification 
